\title{
Parametric inference for nonsynchronously observed diffusion processes in the presence of market microstructure noise
}

\author{
TEPPEI OGIHARA
}

The Institute of Statistical Mathematics, 10-3 Midori-cho, Tachikawa, Tokyo 190-8562, Japan.

E-mail: ogihara@ism.ac.jp

PRESTO, Japan Science and Technology Agency

School of Multidisciplinary Sciences, SOKENDAI (The Graduate University for Advanced Studies)

We study parametric inference for diffusion processes when observations occur nonsynchronously and are contaminated by market microstructure noise. We construct a quasi-likelihood function and study asymptotic mixed normality of maximum-likelihood- and Bayes-type estimators based on it. We also prove the local asymptotic normality of the model and asymptotic efficiency of our estimator when the diffusion coefficients are deterministic and noise follows a normal distribution. We conjecture that our estimator is asymptotically efficient even when the latent process is a general diffusion process. An estimator for the quadratic covariation of the latent process is also constructed. Some numerical examples show that this estimator performs better compared to existing estimators of the quadratic covariation.

Keywords: asymptotic efficiency; Bayes-type estimation; diffusion processes; local asymptotic normality; market microstructure noise; maximum-likelihood-type estimation; nonsynchronous observations; parametric estimation

\section{Contents}

1. Introduction $\ldots \ldots \ldots \ldots \ldots \ldots \ldots \ldots \ldots$

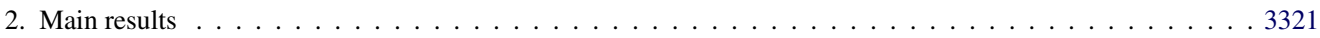

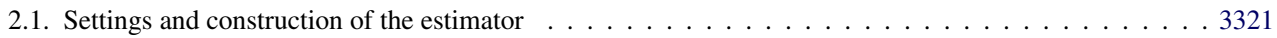

2.2. Asymptotic mixed normality of the maximum-likelihood-type estimator . . . . . . . . . . . . 3325

2.3. On the LAMN property and asymptotic efficiency of the estimator . . . . . . . . . . . . . . . . 3328

2.4. A Bayes-type estimator and convergence of moments of estimation errors . . . . . . . . . . . . 3329

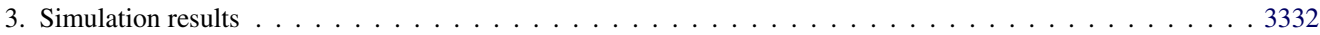

4. Asymptotically equivalent representation of the quasi-likelihood function . . . . . . . . . . . . 3336

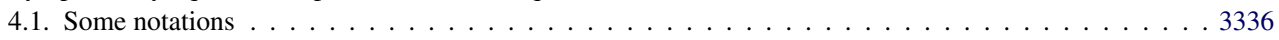

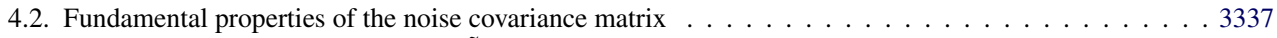

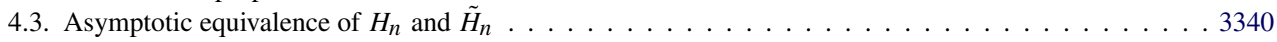

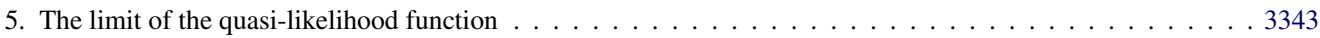

6. Identifiability of the model . . . . . . . . . . . . . . . . . . . . . . . . . . . 3354

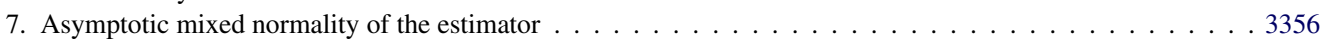

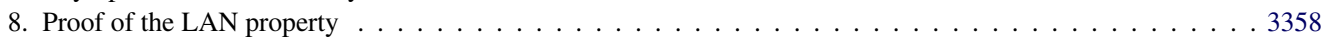

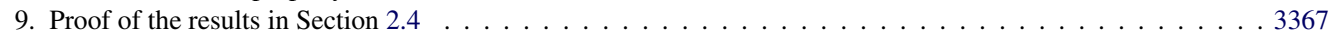

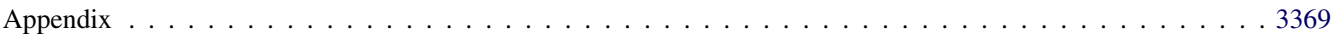

A.1. Results from linear algebra . . . . . . . . . . . . . . . . . . . . . . . . 3369

1350-7265 C 2018 ISI/BS 


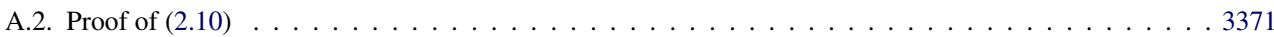

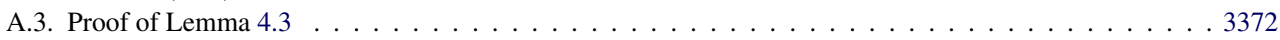

A.4. An additional lemma . . . . . . . . . . . . . . . . . . . . . . . . . . . . . 3380

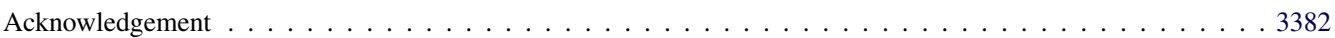

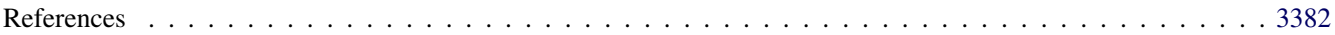

\section{Introduction}

Analysis of volatility and covariation is one of the most important subjects in the study of risk management of financial assets. Studies of high-frequency financial data are increasingly significant as high-frequency financial data become increasingly available and computing technology develops. While realized volatility has been studied as a consistent estimator of integrated volatility at high-frequency limits, estimators of covariation of two securities are also important. The realized covariance, a natural extension of the realized volatility, is a consistent estimator of integrated covariation in ideal settings.

However, there are two significant problems in empirical analysis, one of which is the existence of observation noise. When we model stock price data by a continuous stochastic process, we should assume that the observations are contaminated by additional noise as a way to explain empirical evidence. Consistent estimators of volatility under the presence of microstructure noise are investigated - for example, in Zhang, Mykland, and Aït-Sahalia [31], Barndorff-Nielsen et al. [3], and Podolskij and Vetter [26] — by using various data-averaging or resampling methods to reduce the influence of noise. The other significant problem is that of nonsynchronous observation, namely, that we observe prices of different securities at different time points. The realized covariance has serious bias under models of nonsynchronous observations, though we can calculate the estimator by using some simple "synchronization" methods such as linear interpolation or the "previous tick" methods. Hayashi and Yoshida [15-17] and Malliavin and Mancino [22, 23 ] independently constructed consistent estimators for statistical models of diffusion processes with nonsynchronous observations. There are also studies of covariation estimation under the simultaneous presence of microstructure noise and nonsynchronous observations. We refer interested readers to Barndorff-Nielsen et al. [4] for a kernel based method; Christensen, Kinnebrock, and Podolskij [7], Christensen, Podolskij, and Vetter [8] for the modulated realised covariance and a pre-averaged Hayashi-Yoshida estimator; Aït-Sahalia, Fan, and Xiu [2] for a method using the maximum likelihood estimator of a model with constant diffusion coefficients; and Bibinger et al. [5] for a technique employing the local method of moments.

While the above studies concern estimators under non- or semi-parametric settings, there are also studies about parametric inference of diffusion processes with high-frequency observations. Genon-Catalot and Jacod [11] constructed a quasi-likelihood function and studied an estimator that maximizes it. Gloter and Jacod [13] studied an estimator based on a quasi-likelihood function with noisy observations. Ogihara and Yoshida [25] studied a maximum-likelihood-type estimator and a Bayes-type estimator on nonsynchronous observations without market microstructure noise.

One advantage of maximum-likelihood- and Bayes-type estimators is that they are asymptotically efficient in many models. If a statistical model has the local asymptotic mixed normality 
(LAMN) property, then the results in Jeganathan [20,21] ensure that asymptotic variance of estimators cannot be smaller than a certain lower bound. When some estimator attains this bound, it is called asymptotically efficient. For parametric estimation of diffusion processes on fixed intervals, Gobet [14] proved the LAMN property of the statistical model having equidistant observations, and an estimator in [11] is asymptotically efficient. Ogihara [24] proved the LAMN property and asymptotic efficiency of estimators for the setting of [25]. Gloter and Jacod [12] proved the local asymptotic normality (LAN) property for a statistical model with market microstructure noise when diffusion coefficients are deterministic, and the estimator by Gloter and Jacod [13] is asymptotically efficient. There are few studies about the efficiency of estimators that assume the presence of market microstructure noise and nonsynchronous observations. One exception is Bibinger et al. [5], who showed a lower bound of asymptotic variance of estimators in semi-parametric Cramér-Rao sense. We need the LAN or LAMN property of the statistical model to obtain asymptotic efficiency of a parametric model. To the best of our knowledge, this has not been studied for statistical models of noisy, nonsynchronous observations.

This paper examines consistency and asymptotic mixed normality of a maximum-likelihoodtype estimator and a Bayes-type estimator based on a quasi-likelihood function, under the simultaneous presence of market microstructure noise and nonsynchronous observations. We also study the LAN property of this model when diffusion coefficients are deterministic, as well as the asymptotic efficiency of our estimators. We expect that our estimators are asymptotically efficient in the general cases. However, it is further difficult to obtain LAMN properties for models of general diffusions. This does not seem to have been obtained even for noisy, equidistant observations, and is left as future work. We will see by simulation that sample variance of the estimation error of our estimator is better than that of existing estimators for some examples in Section 3. These results ensure that our estimator not only is the theoretical best for asymptotic behavior, but also works well in practical finite samplings.

Our study has several advantages in addition to the above arguments regarding asymptotic efficiency.

(i) Our model also allows observation noise that follows a non-Gaussian distribution. We use a quasi-likelihood function for Gaussian noise, but our method is robust enough to allow misspecification of the noise distribution.

(ii) Since we obtain the results regarding asymptotic behaviors of the quasi-likelihood function as a byproduct, many applications become available from the theory of maximumlikelihood-type estimation. For example, we can construct a theory of the likelihood ratio test and one-step estimators as an immediate application. Further, the theory of information criteria is expected to follow from our results of quasi-likelihood functions.

(iii) Our settings contain random sampling schemes where the maximum length of observation intervals is not bounded by any constant multiplication of the minimum length. This is the case for some significant random sampling schemes, such as samplings based on Poisson or Cox processes. Our model encompasses such natural sampling schemes.

To obtain asymptotic mixed normality of our estimator, we investigate asymptotic behaviors of a quasi-likelihood function of noisy, nonsynchronous observations. To this end, we need to specify the limit of some matrix trace related to a ratio of covariance matrices for two different values of parameters, as appearing in (5.2). The inverse of the covariance matrix of observation noise has nontrivial off-diagonal elements, and so the inverse of the covariance matrix of 
observations is far from a diagonal matrix. This phenomenon is essentially different from the case of synchronous observations without noise (where the covariance matrix of observations is diagonal), and the case of nonsynchronous observations without noise (where the inverse of the covariance matrix is not a diagonal matrix but is "close" to being one).

In a model of noisy, synchronous observations, the covariance matrix of a latent process is asymptotically equivalent to a unit matrix of the appropriate size, and is therefore simultaneously diagonalizable with the noise covariance. Gloter and Jacod [12,13] used these facts and closed expressions for the eigenvalues of the noise covariance to identify the limit of the quasi-likelihood function, but we cannot apply their idea because our sampling scheme is irregular and so not well approximated by a unit matrix. Further, the sizes of the covariance matrices are different for different components of the process, which follows from nonsynchronousness. In this paper, we deduce an asymptotically equivalent transform of the trace of the ratio of covariance matrices. This transform changes sizes of matrices and matrix elements into local averages, and arises from specific properties of the noise covariance matrix. We will see these results in Sections 4 and 5.

The remainder of this paper is organized as follows. In Section 2, we describe our detailed settings and main results. We propose a quasi-likelihood function for models with noisy, nonsynchronous observations, and construct a maximum-likelihood-type estimator based on it. We introduce asymptotic mixed normality of our estimator and results about asymptotic efficiency in Section 2.2. Section 2.3 contains results about the LAN property of our model and the asymptotic efficiency of our estimator, and Section 2.4 is devoted to results about Bayes-type estimators and convergence of moments of estimators. Polynomial-type large deviation inequalities, introduced in Yoshida [29,30], are key to deducing these results. In Section 3 we will examine simulation results of our estimator for a simple example where the latent process is a Wiener process. We also construct an estimator of the quadratic covariation and compare the performance of our estimator with that of other estimators. The remaining sections are devoted to a proof of the main results. Section 4 introduces an asymptotically equivalent expression of the quasi-likelihood function. This expression is useful for deducing asymptotic properties of the quasi-likelihood function in Section 5. We also need some results on identifiability of the model to obtain consistency of the maximum-likelihood-type estimator. These are discussed in Section 6. Section 7 shows asymptotic mixed normality of our estimator. The LAN property of the model for deterministic diffusion coefficients is obtained in Section 8. Section 9 contains a proof of results regarding the Bayes-type estimator and the convergence of moments of estimators.

\section{Main results}

\subsection{Settings and construction of the estimator}

Let $\left(\Omega^{(0)}, \mathcal{F}^{(0)}, P^{(0)}\right)$ be a probability space with a filtration $\mathbf{F}^{(0)}=\left\{\mathcal{F}_{t}^{(0)}\right\}_{0 \leq t \leq T}$. We consider a two-dimensional $\mathbf{F}^{(0)}$-adapted process $Y=\left\{Y_{t}\right\}_{0 \leq t \leq T}$ satisfying the stochastic integral equation:

$$
Y_{t}=Y_{0}+\int_{0}^{t} \mu_{s} d s+\int_{0}^{t} b\left(s, X_{s}, \sigma_{*}\right) d W_{s}, \quad t \in[0, T],
$$


where $\left\{W_{t}\right\}_{0 \leq t \leq T}$ is a $d_{1}$-dimensional standard $\mathbf{F}^{(0)}$-Wiener process, $b=\left(b^{i j}\right)_{1 \leq i \leq 2,1 \leq j \leq d_{1}}$ is a Borel function, $\mu=\left\{\mu_{t}\right\}_{0 \leq t \leq T}$ is a locally bounded $\mathbf{F}^{(0)}$-adapted process with values in $\mathbb{R}^{2}$, and $X=\left\{X_{t}\right\}_{0 \leq t \leq T}$ is a continuous $\mathbf{F}^{(0)}$-adapted processes with values in $O$, an open subset of $\mathbb{R}^{d_{2}}$ with $d_{2} \in \mathbb{N}$. We consider market microstructure noise $\left\{\varepsilon_{i}^{n, k}\right\}_{n \in \mathbb{N}, i \in \mathbb{Z}_{+}, k=1,2}$ as an independent sequence of random variables on another probability space $\left(\Omega^{(1)}, \mathcal{F}^{(1)}, P^{(1)}\right)$. We assume that $\mathcal{F}^{(1)}=\mathfrak{B}\left(\left(\varepsilon_{i}^{n, k}\right)_{n, k, i}\right)$ and that the distribution of $\varepsilon_{j}^{n, k}$ does not depend on $j$, where $\mathfrak{B}(S)$ denotes the minimal $\sigma$-field such that any element of $S$ is $\mathfrak{B}(S)$-measurable for a set $S$ of random variables. We use the same notation $\mathfrak{B}(S)$ for a similarly defined $\sigma$-field for a set $S$ of measurable sets. We consider a product probability space $(\Omega, \mathcal{F}, P)$, where $\Omega=\Omega^{(0)} \times \Omega^{(1)}$, $\mathcal{F}=\mathcal{F}^{(0)} \otimes \mathcal{F}^{(1)}$, and $P=P^{(0)} \otimes P^{(1)}$.

We assume that the observations of processes occur in a nonsynchronous manner and are contaminated by market microstructure noise, that is, we observe the vectors $\left\{\tilde{Y}_{i}^{k}\right\}_{0 \leq i \leq \mathbf{J}_{k, n}, k=1,2}$ and $\left\{\tilde{X}_{j}^{k}\right\}_{0 \leq j \leq \mathbf{J}_{k, n}^{\prime}, 1 \leq k \leq d_{2}}$, where $\left\{S_{i}^{n, k}\right\}_{i=0}^{\mathbf{J}_{k, n}}$ and $\left\{T_{j}^{n, k}\right\}_{j=0}^{\mathbf{J}_{k, n}^{\prime}}$ are random times in $\left(\Omega^{(0)}, \mathcal{F}^{(0)}\right)$, $\left\{\eta_{j}^{n, k}\right\}_{j \in \mathbb{Z}_{+}, 1 \leq k \leq d_{2}}$ is a random sequence on $(\Omega, \mathcal{F})$, and

$$
\tilde{Y}_{i}^{k}=Y_{S_{i}^{n, k}}^{k}+\varepsilon_{i}^{n, k}, \quad \tilde{X}_{j}^{k}=X_{T_{j}^{n, k}}^{k}+\eta_{j}^{n, k}
$$

Our goal is to estimate the true value $\sigma_{*}$ of the parameter from nonsynchronous, noisy observations $\left\{S_{i}^{n, k}\right\}_{0 \leq i \leq \mathbf{J}_{k, n}, k=1,2},\left\{T_{j}^{n, k}\right\}_{0 \leq j \leq \mathbf{J}_{k, n}^{\prime}, 1 \leq k \leq d_{2}},\left\{\tilde{Y}_{i}^{k}\right\}_{0 \leq i \leq \mathbf{J}_{k, n}, k=1,2}$, and $\left\{\tilde{X}_{j}^{k}\right\}_{0 \leq j \leq \mathbf{J}_{k, n}^{\prime}, 1 \leq k \leq d_{2}}$.

By setting $d_{2}=2, X_{t} \equiv Y_{t}, \mu_{t}=\mu\left(t, Y_{t}\right), S_{i}^{n, k} \equiv T_{j}^{n, k}$, and $\eta_{j}^{n, k} \equiv \varepsilon_{i}^{n, k}$, our model contains the case where the latent process $Y$ is a diffusion process satisfying a stochastic differential equation

$$
d Y_{t}=\mu\left(t, Y_{t}\right) d t+b\left(t, Y_{t}, \sigma_{*}\right) d W_{t}, \quad t \in[0, T],
$$

and $Y$ is observed in a nonsynchronous manner with noise. This model is of particular interest, but our results are also be applied to more general models (2.1).

Remark 2.1. Stochastic volatility models are significant models for modeling stock prices. Unfortunately, our settings are not applied to hidden Markov models including stochastic volatility models because we require (possibly noisy) observations of process $X$. However, we hope that our results give an essential idea to deal with noisy, nonsynchronous observations, and therefore we can construct an estimator for stochastic volatility models by replacing our quasi-likelihood function. We have left it for future works.

For a vector $x=\left(x_{1}, \ldots, x_{k}\right)$, we denote $\partial_{x}^{l}=\left(\frac{\partial^{l}}{\partial_{x_{i_{1}} \cdots \partial_{x_{i_{l}}}}}\right)_{i_{1}, \ldots, i_{l}=1}^{k}$. We assume the true value $\sigma_{*}$ of the parameter is contained in a bounded open set $\Lambda \subset \mathbb{R}^{d}$ that satisfies Sobolev's inequality; that is, for any $p>d$, there exists $C>0$ such that $\sup _{\sigma \in \Lambda}|u(\sigma)| \leq$ $C \sum_{k=0,1}\left(\int_{\Lambda}\left|\partial_{\sigma}^{k} u(\sigma)\right|^{p} d \sigma\right)^{1 / p}$ for any $u \in C^{1}(\Lambda)$. This is the case when $\Lambda$ has a Lipshitz boundary. See Adams and Fournier [1] for more details.

Let $\Pi_{n}=\left(\left\{S_{i}^{n, k}\right\}_{k, i},\left\{T_{j}^{n, k}\right\}_{k, j}\right)$ and $\left\{\mathcal{G}_{t}\right\}_{0 \leq t \leq T}$ be a filtration of $(\Omega, \mathcal{F}, P)$ given by

$$
\mathcal{G}_{t}=\mathcal{F}_{t}^{(0)} \vee \mathfrak{B}\left(\left\{\Pi_{n}\right\}_{n}\right) \vee \mathfrak{B}\left(A \cap\left\{S_{i}^{n, k} \leq t\right\} ; A \in \mathfrak{B}\left(\varepsilon_{i}^{n, k}\right), k \in\{1,2\}, i \in \mathbb{Z}_{+}, n \in \mathbb{N}\right),
$$


where $\mathcal{H}_{1} \vee \mathcal{H}_{2}$ denotes the minimal $\sigma$-field which contains $\sigma$-fields $\mathcal{H}_{1}$ and $\mathcal{H}_{2}$. Let $E_{\Pi}[\mathbf{X}]=$ $E\left[\mathbf{X} \mid\left\{\Pi_{n}\right\}_{n}\right]$ for a random variable $\mathbf{X}$. We assume that $\mathcal{F}_{T}^{(0)}$ and $\mathfrak{B}\left(\left\{\Pi_{n}\right\}_{n}\right)$ are independent. Moreover, we assume that there exist positive constants $v_{1, *}$ and $v_{2, *}$ such that $\eta_{j}^{n, k} 1_{\left\{T_{j}^{n, k} \leq t\right\}}$ is $\mathcal{G}_{t}$-measurable, $E\left[\varepsilon_{i}^{n, k}\right]=0$, and $E\left[\left(\varepsilon_{i}^{n, k}\right)^{2}\right]=v_{k, *}$ for any $n, k, i, j, t$, where $1_{A}$ is the indicator function for a set $A, \delta_{i j}$ is Kronecker's delta. We also assume that the distribution of $Y_{0}$ does not depend on $\sigma_{*}, v_{1, *}$, nor $v_{2, *}$.

Now we construct the quasi-likelihood function. We apply the idea of Gloter and Jacod [13] to our construction of a quasi-likelihood function; that is, we divide the whole observation interval $[0, T]$ into equidistant subdivisions and construct quasi-likelihood functions for each interval as follows. Let $\left\{b_{n}\right\}_{n \in \mathbb{N}}$ and $\left\{k_{n}\right\}_{n \in \mathbb{N}}$ be sequences of positive numbers satisfying $b_{n} \geq 1$, $k_{n} \leq b_{n}, b_{n} \rightarrow \infty, k_{n} b_{n}^{-1 / 2-\varepsilon} \rightarrow \infty$, and $k_{n} b_{n}^{-2 / 3+\varepsilon} \rightarrow 0$ as $n \rightarrow \infty$ for some $\varepsilon>0$. We will assume in Assumption (A2) a relation between $b_{n}$ and our sampling scheme, which implies that $b_{n}$ represents the order of observation frequency. Let $\ell_{n}=\left[b_{n} k_{n}^{-1}\right], s_{0}=0, s_{m}=T \ell_{n}^{-1} m$, $b^{k}(t, x, \sigma)=\left(b^{k j}(t, x, \sigma)\right)_{j=1}^{d_{1}}, K_{0}^{k}=-1$, and $K_{m}^{k}=\#\left\{i \in \mathbb{N} ; S_{i}^{n, k}<s_{m}\right\}$ for $k \in\{1,2\}$ and $1 \leq m \leq \ell_{n}$. Moreover, let $k_{m}^{j}=K_{m}^{j}-K_{m-1}^{j}-1, \bar{k}_{n}=\max _{m, j} k_{m}^{j}, \underline{\mathrm{k}}_{n}=\min _{m, j} k_{m}^{j}$,

$$
\begin{aligned}
& I_{i, m}^{k}=\left[S_{i+K_{m-1}^{k}, k}^{n, k} S_{i+1+K_{m-1}^{k}}^{n, k}\right), \quad \tilde{Y}^{k}\left(I_{i, m}^{k}\right)=\tilde{Y}_{i+1+K_{m-1}^{k}}^{k}-\tilde{Y}_{i+K_{m-1}^{k}}^{k}, \\
& \hat{X}_{m}=\left(\#\left\{j ; T_{j}^{n, k} \in\left[s_{m-1}, s_{m}\right)\right\}^{-1} \sum_{j ; T_{j}^{n, k} \in\left[s_{m-1}, s_{m}\right)} \tilde{X}_{j}^{k}\right)_{1 \leq k \leq d_{2}},
\end{aligned}
$$

and $b_{m^{\prime}}^{j}(\sigma)=b^{j}\left(s_{m^{\prime}-1}, \hat{X}_{m^{\prime}-1}, \sigma\right)$ for $1 \leq m \leq \ell_{n}, 2 \leq m^{\prime} \leq \ell_{n}, j \in\{1,2\}$ and $1 \leq i \leq k_{m}^{j}$. Then, roughly speaking, we have the following approximations of conditional covariance of observations:

$$
\begin{aligned}
E\left[\tilde{Y}^{k}\left(I_{i, m}^{k}\right) \tilde{Y}^{k}\left(I_{i^{\prime}, m}^{k}\right) \mid \mathcal{G}_{s_{m-1}}\right] & \approx\left(\left|b_{m}^{k}\right|^{2}\left|I_{i, m}^{k}\right|+2 v_{k, *}\right) \delta_{i i^{\prime}}-v_{k, *} 1_{\left\{\left|i-i^{\prime}\right|=1\right\}}, \\
E\left[\tilde{Y}^{1}\left(I_{i^{\prime \prime}, m}^{1}\right) \tilde{Y}^{2}\left(I_{i^{\prime \prime \prime}, m}^{2}\right) \mid \mathcal{G}_{s_{m-1}}\right] & \approx b_{m}^{1} \cdot b_{m}^{2}\left|I_{i^{\prime \prime}, m}^{1} \cap I_{i^{\prime \prime \prime}, m}^{2}\right|
\end{aligned}
$$

for any intervals $I_{i, m}^{k}, I_{i^{\prime}, m}^{k}, I_{i^{\prime \prime}, m}^{1}, I_{i^{\prime \prime \prime}, m}^{2}$.

Let $T$ denotes the transpose operator for matrices (and vectors), $M(l)=\left\{2 \delta_{i_{1} i_{2}}-\right.$ $\left.1_{\left\{\left|i_{1}-i_{2}\right|=1\right\}}\right\}_{i_{1}, i_{2}=1}^{l}$ for $l \in \mathbb{N}, M_{j, m}=M\left(k_{m}^{j}\right)$ for $1 \leq j \leq 2$. Based on the relation (2.4), we define a quasi-log-likelihood function $H_{n}(\sigma, v)$ by

$$
H_{n}(\sigma, v)=-\frac{1}{2} \sum_{m=2}^{\ell_{n}} Z_{m}^{\top} S_{m}^{-1}(\sigma, v) Z_{m}-\frac{1}{2} \sum_{m=2}^{\ell_{n}} \log \operatorname{det} S_{m}(\sigma, v),
$$

where $Z_{m}=\left(\left(\tilde{Y}^{1}\left(I_{i, m}^{1}\right)\right)_{1 \leq i \leq k_{m}^{1}}^{\top},\left(\tilde{Y}^{2}\left(I_{i, m}^{2}\right)\right)_{1 \leq i \leq k_{m}^{2}}^{\top}\right)^{\top}$ and

$S_{m}(\sigma, v)=\left(\begin{array}{cc}\left\{\left|b_{m}^{1}\right|^{2}\left|I_{i, m}^{1}\right| \delta_{i i^{\prime}}\right\}_{i i^{\prime}} & \left\{b_{m}^{1} \cdot b_{m}^{2}\left|I_{i, m}^{1} \cap I_{j, m}^{2}\right|\right\}_{i j} \\ \left\{b_{m}^{1} \cdot b_{m}^{2}\left|I_{i, m}^{1} \cap I_{j, m}^{2}\right|\right\}_{j i} & \left\{\left|b_{m}^{2}\right|^{2}\left|I_{j, m}^{2}\right| \delta_{j j^{\prime}}\right\}_{j j^{\prime}}\end{array}\right)+\left(\begin{array}{cc}v_{1} M_{1, m} & 0 \\ 0 & v_{2} M_{2, m}\end{array}\right)$

for $v=\left(v_{1}, v_{2}\right)$. 
Remark 2.2. Though such a local Gaussian quasi-log-likelihood function seems valid only when observation noise $\varepsilon_{i}^{n, k}$ follows a Gaussian distribution, asymptotic properties of the maximum likelihood estimator are robust enough to allow non-Gaussian noise. Hence, we can use the same quasi-likelihood function for general noise.

Remark 2.3. We used subdivisions of $[0, T]$ for the construction of $H_{n}$ because of technical issues related to deducing the limit of $H_{n}$. Since the diffusion coefficient $b$ in $S_{m}$ is fixed, matrix properties of $M_{j, m}$ introduced in Section 4.2 can be used to deduce the limit of $H_{n}$. On the other hand, such a construction of $H_{n}$ also contributes to reducing the calculation time of the maximum-likelihood-type estimator because the size of $S_{m}$ is $O\left(k_{n}\right)$ while the size of the covariance matrix of all observations is $O\left(b_{n}\right)$.

Remark 2.4. In [13], $k_{n}$ is taken so that $n^{1 / 2} k_{n}^{-1} \rightarrow 0$ and $k_{n} n^{-3 / 4} \rightarrow 0$. Our rate $b_{n}^{2 / 3}$ for the upper bound of $k_{n}$ is a little bit worse because of some technical issue (for equidistance observations, we have $b_{n} \equiv n$ ). When we investigate asymptotic behaviors of the maximumlikelihood-type estimator, we deal with some supremum estimates for the $\sigma$ of quasi-likelihood ratios. Unlike the one-dimensional settings of [13], our multidimensional setting requires some properties to deal with the supremum. We use Sobolev's inequality here for this purpose. Then we need an additional moment estimate for quasi-likelihood ratios, which causes a worse rate of $k_{n}$. See the proofs of Lemmas 4.3 and 4.4 for details.

To construct the maximum-likelihood-type estimator $\hat{\sigma}_{n}$ for the parameter $\sigma$, we need estimators for the unknown noise variance $v_{*}=\left(v_{1, *}, v_{2, *}\right)$. We assume the following condition.

Assumption (V). There exist estimators $\left\{\hat{v}_{n}\right\}_{n \in \mathbb{N}}$ of $v_{*}$ such that $\hat{v}_{n} \geq 0$ almost surely and $\left\{b_{n}^{1 / 2}\left(\hat{v}_{n}-v_{*}\right)\right\}_{n \in \mathbb{N}}$ is tight.

For example, $\hat{v}_{n}=\left(\hat{v}_{n, k}\right)_{k=1}^{2}$ with $\hat{v}_{n, k}=\left(2 \mathbf{J}_{k, n}\right)^{-1} \sum_{i}\left(\tilde{Y}_{i}^{k}-\tilde{Y}_{i-1}^{k}\right)^{2}$ satisfies (V) if $\left\{b_{n} \mathbf{J}_{k, n}^{-1}\right\}_{n}$ is tight for $k=1,2$ and $\sup _{n, k, i} E\left[\left(\varepsilon_{i}^{n, k}\right)^{4}\right]<\infty$.

Let $\operatorname{clos}(A)$ be the closure of a set $A$. A maximum-likelihood-type estimator $\hat{\sigma}_{n}$ is a random variable satisfying

$$
H_{n}\left(\hat{\sigma}_{n}, \hat{v}_{n}\right)=\max _{\sigma \in \operatorname{clos}(\Lambda)} H_{n}\left(\sigma, \hat{v}_{n}\right) .
$$

We study asymptotic mixed normality and asymptotic efficiency of the estimator in the following subsections.

Remark 2.5. We can also construct a simultaneous maximum-likelihood-type estimator $\left(\bar{\sigma}_{n}, \bar{v}_{n}\right)$ satisfying $H_{n}\left(\bar{\sigma}_{n}, \bar{v}_{n}\right)=\max _{\sigma, v} H_{n}(\sigma, v)$. However, it is valid only when the observation noise $\varepsilon_{i}^{n, k}$ follows a normal distribution. Our interest is on estimating the parameter $\sigma$ of the latent process, and so the assumptions for observation noise should be reduced as much as possible. Therefore, the nonparametric estimator $\hat{v}_{n}$ is more suitable for our purpose. 


\subsection{Asymptotic mixed normality of the maximum-likelihood-type estimator}

In the rest of this section, we state our main theorems. Proofs of these results are left to Sections 4-9. In this subsection, we describe the asymptotic mixed normality of the maximumlikelihood-type estimator $\hat{\sigma}_{n}$.

We first describe assumptions for the theorem. Assumption (A1) is a sequence of assumptions on the latent processes $Y$ and $X$ and observation noise $\varepsilon_{i}^{n, k}$ and $\eta_{j}^{n, k}$. We denote by $\mathcal{E}_{l}$ the unit matrix of size $l$.

Assumption (A1). 1. For $0 \leq 2 i+j \leq 4$ and $0 \leq k \leq 4$, the derivatives $\partial_{t}^{i} \partial_{x}^{j} \partial_{\sigma}^{k} b(t, x, \sigma)$ exist on $[0, T] \times O \times \Lambda$ and have continuous extensions on $[0, T] \times O \times \operatorname{clos}(\Lambda)$.

2. $b b^{\top}(t, x, \sigma)$ is positive definite for $(t, x, \sigma) \in[0, T] \times O \times \operatorname{clos}(\Lambda)$.

3. $\sup _{n, k, i} E\left[\left(\varepsilon_{i}^{n, k}\right)^{q}\right]<\infty$ for any $q>0$.

4. $\mu_{t}$ is optional and locally bounded (locally in time), that is, there exists an increasing sequence $\left\{T_{l}\right\}_{l}$ of stopping times such that $\lim _{l \rightarrow \infty} T_{l}=T$ a.s. and $\left\{\mu_{t \wedge T_{l}}\right\}_{0 \leq t \leq T}$ is bounded for each $l$.

5. $b_{n}^{-\varepsilon} \max _{m, k}\left(E_{\Pi}\left[\left|\ell_{n}^{1 / 2} \mathbf{T}_{m, k}\right|^{q} \vee \mid \ell_{n} E\left[\mathbf{T}_{m, k}\left|\mathcal{G}_{S_{m-1}}\right|^{q}\right]\right) \rightarrow^{p} 0\right.$ as $n \rightarrow \infty$ for any $q>0$ and $\varepsilon>0$, where $\mathbf{T}_{m, k}=\#\left\{j ; T_{j}^{n, k} \in\left[s_{m-1}, s_{m}\right)\right\}^{-1} \sum_{j ; T_{j}^{n, k} \in\left[s_{m-1}, s_{m}\right)} \eta_{j}^{n, k}$.

6. There exist progressively measurable processes $\left\{b_{t}^{(j)}\right\}_{0 \leq t \leq T, 0 \leq j \leq 1}$ and $\left\{\hat{b}_{t}^{(j)}\right\}_{0 \leq t \leq T, 0 \leq j \leq 1}$ such that $\sup _{t} E\left[\left|b_{t}^{(j)}\right|^{q} \vee\left|\hat{b}_{t}^{(j)}\right|^{q}\right]<\infty, \sup _{s<t} E\left[\left|b_{t}^{(j)}-b_{s}^{(j)}\right|^{q} \vee\left|\hat{b}_{t}^{(j)}-\hat{b}_{s}^{(j)}\right|^{q}\right]^{1 / q}(t-s)^{-1 / 2}<$ $\infty$ for $0 \leq j \leq 1$ and $q>0$, and

$$
X_{t}=X_{0}+\int_{0}^{t} b_{s}^{(0)} d s+\int_{0}^{t} b_{s}^{(1)} d W_{s}, \quad b_{t}^{(1)}=b_{0}^{(1)}+\int_{0}^{t} \hat{b}_{s}^{(0)} d s+\int_{0}^{t} \hat{b}_{s}^{(1)} d W_{s}
$$

for $t \in[0, T]$.

Assumption (A1) captures somewhat standard assumptions and whether it holds can easily verified in practical settings. Roughly speaking, point 5 of (A1) is satisfied if the summation of $\eta_{j}^{n, k}$ is of an order equivalent to the square root of the number of $\eta_{j}^{n, k}$. This is satisfied under certain independency, martingale conditions or mixing conditions of $\eta_{j}^{n, k}$. If $\left\{\eta_{j}^{n, k}\right\}_{j}$ is a sequence of centered, independent and identically distributed random variables and the sequence has finite moments, then $E_{\Pi}\left[\left|\mathbf{T}_{m, k}\right|^{q}\right]=O_{p}\left(\#\left\{j ; T_{j}^{n, k} \in\left[s_{m-1}, s_{m}\right)\right\}^{-q / 2}\right)$. Then, point 5 of (A1) is satisfied if sampling frequency of $\left\{T_{j}^{n, k}\right\}$ is of order $b_{n}$ and $E\left[\eta_{j}^{n, k} 1_{\left\{T_{j}^{n, k}>s_{m-1}\right\}} \mid \mathcal{G}_{s_{m-1}}\right]=0$. Decomposition of $X$ in point 6 of (A1) is used to deduce asymptotically equivalent representation of $H_{n}$ where the diffusion coefficient $b\left(t, X_{t}, \sigma_{*}\right)$ is replaced by $b\left(s_{m-1}, X_{s_{m-1}}, \sigma_{*}\right)$. Detailed semimartingale decomposition is required to estimate the difference $b\left(t, X_{t}, \sigma_{*}\right)-b\left(s_{m-1}, X_{s_{m-1}}, \sigma_{*}\right)$.

In the following, we assume some conditions about our sampling scheme. For $\eta \in(0,1 / 2)$, let $\mathcal{S}_{\eta}$ be the set of all sequences $\left\{\left[s_{n, l}^{\prime}, s_{n, l}^{\prime \prime}\right)\right\}_{n \in \mathbb{N}, 1 \leq l \leq L_{n}}$ of intervals on $[0, T]$ satisfying $\left\{L_{n}\right\}_{n} \subset \mathbb{N}$, $\left[s_{n, l_{1}}^{\prime}, s_{n, l_{2}}^{\prime \prime}\right) \cap\left[s_{n, l_{2}}^{\prime}, s_{n, l_{2}}^{\prime \prime}\right)=\varnothing$ for $n, l_{1} \neq l_{2}, \inf _{n, l}\left(b_{n}^{1-\eta}\left(s_{n, l}^{\prime \prime}-s_{n, l}^{\prime}\right)\right)>0$, and $\sup _{n, l}\left(b_{n}^{1-\eta}\left(s_{n, l}^{\prime \prime}-\right.\right.$ $\left.\left.s_{n, l}^{\prime}\right)\right)<\infty$. Let $r_{n}=\max _{i, k}\left|S_{i}^{n, k}-S_{i-1}^{n, k}\right|$ and $\underline{\mathrm{r}}_{n}=\min _{i, k}\left|S_{i}^{n, k}-S_{i-1}^{n, k}\right|$. 
Assumption (A2). There exist $\eta \in(0,1 / 2), \kappa>0, \dot{\eta} \in(0,1]$ and positive-valued stochastic processes $\left\{a_{t}^{j}\right\}_{t \in[0, T], j=1,2}$ such that $\sup _{t \neq s}\left(\left|a_{t}^{j}-a_{s}^{j}\right| /|t-s|^{\dot{\eta}}\right)<\infty$ almost surely, $b_{n}^{-1 / 2+\kappa} \times$ $k_{n}\left(b_{n}^{-1} k_{n}\right)^{\dot{\eta}} \rightarrow 0$ and

$$
k_{n} b_{n}^{-1 / 2+\kappa} \max _{1 \leq l \leq L_{n}}\left|b_{n}^{-1}\left(s_{n, l}^{\prime \prime}-s_{n, l}^{\prime}\right)^{-1} \#\left\{i ;\left[S_{i-1}^{n, j}, S_{i}^{n, j}\right) \subset\left[s_{n, l}^{\prime}, s_{n, l}^{\prime \prime}\right)\right\}-a_{s_{n, l}^{\prime}}^{j}\right| \stackrel{p}{\rightarrow} 0
$$

as $n \rightarrow \infty$ for $j=1,2$ and $\left\{\left[s_{n, l}^{\prime}, s_{n, l}^{\prime \prime}\right)\right\}_{1 \leq l \leq L_{n}, n \in \mathbb{N}} \in \mathcal{S}_{\eta}$. Moreover, $\left(r_{n} b_{n}^{1-\varepsilon}\right) \vee\left(b_{n}^{-1-\varepsilon} \underline{\mathbf{r}}_{n}^{-1}\right) \rightarrow^{p} 0$ for any $\varepsilon>0$.

In particular, Assumption (A2) implies $b_{n}^{-1} \mathbf{J}_{j, m} \rightarrow^{p} \int_{0}^{T} a_{t}^{j} d t$ and $\max _{m} \mid T^{-1} k_{n}^{-1} k_{m}^{j}-$ $a_{s_{m-1}}^{j} \mid \rightarrow^{p} 0$ as $n \rightarrow \infty$. Roughly speaking, (A2) shows the law of large numbers for sampling schemes in any local time intervals. In the proof of Lemma 5.2, we will see that some properties of $M_{j, m}$ enable us to replace $\left|I_{k}^{j}\right|$ in $S_{m}(\sigma, v)$ by the local average in asymptotics. Then (A2) leads to the limit of $H_{n}$.

Example 2.1. Let $\left\{N_{t}^{k}\right\}_{t \geq 0}$ be an exponential $\alpha$-mixing point process with stationary increments for $k=1,2$. Assume that $E\left[\left|N_{1}^{k}\right|^{q}\right]<\infty$ for any $q>0$ and $k=1,2$. Set $S_{i}^{m, k}=\inf \left\{t \geq 0 ; N_{b_{n} t}^{k} \geq\right.$ $i$ \}. Then Rosenthal-type inequalities (Theorem 3 and Lemma 7 in Doukhan and Louhichi [10], or Theorem 4 in [25]) and a similar argument to the proof of Proposition 6 in [25] ensure (A2) with $a_{t}^{j} \equiv E\left[N_{1}^{j}\right]$ (constants). Also, (B2) (defined later) is satisfied if further $k_{n} b_{n}^{-4 / 7+\gamma} \rightarrow 0$ for some $\gamma>0$.

Under the above conditions, we can show convergence of the quasi-likelihood ratio $H_{n}(\sigma$, $\left.\hat{v}_{n}\right)-H_{n}\left(\sigma_{*}, \hat{v}_{n}\right)$. Let $b_{t}=b\left(t, X_{t}, \sigma\right), b_{t, *}=b\left(t, X_{t}, \sigma_{*}\right), \tilde{a}_{t}^{j}=a_{t}^{j} / v_{j, *}$ for $j=1,2$ and

$$
\begin{aligned}
\mathcal{Y}_{1}(\sigma)= & \int_{0}^{T}\left\{\frac{\sum_{j=1}^{2}\left(\left|b_{t}^{j}\right|^{2}-\left|b_{t, *}^{j}\right|^{2}\right)\left(\left|b_{t}^{3-j}\right|^{2} \sqrt{\tilde{a}_{t}^{1} \tilde{a}_{t}^{2}}+\tilde{a}_{t}^{j} \sqrt{\operatorname{det}\left(b_{t} b_{t}^{\top}\right)}\right.}{4 \sqrt{\operatorname{det}\left(b_{t} b_{t}^{\top}\right)}\left(\tilde{a}_{t}^{1}\left|b_{t}^{1}\right|^{2}+\tilde{a}_{t}^{2}\left|b_{t}^{2}\right|^{2}+2 \sqrt{\tilde{a}_{t}^{1} \tilde{a}_{t}^{2} \operatorname{det}\left(b_{t} b_{t}^{\top}\right)}\right)^{1 / 2}}\right. \\
& -\frac{\left(b_{t}^{1} \cdot b_{t}^{2}-b_{t, *}^{1} \cdot b_{t, *}^{2}\right) b_{t}^{1} \cdot b_{t}^{2} \sqrt{\tilde{a}_{t}^{1} \tilde{a}_{t}^{2}}}{2 \sqrt{\operatorname{det}\left(b_{t} b_{t}^{\top}\right)}\left(\tilde{a}_{t}^{1}\left|b_{t}^{1}\right|^{2}+\tilde{a}_{t}^{2}\left|b_{t}^{2}\right|^{2}+2 \sqrt{\tilde{a}_{t}^{1} \tilde{a}_{t}^{2} \operatorname{det}\left(b_{t} b_{t}^{\top}\right)}\right)^{1 / 2}} \\
& -\frac{\left(\tilde{a}_{t}^{1}\left|b_{t}^{1}\right|^{2}+\tilde{a}_{t}^{2}\left|b_{t}^{2}\right|^{2}+2 \sqrt{\left.\tilde{a}_{t}^{1} \tilde{a}_{t}^{2} \operatorname{det}\left(b_{t} b_{t}^{\top}\right)\right)^{1 / 2}}\right.}{2} \\
& \left.+\frac{\left(\tilde{a}_{t}^{1}\left|b_{t, *}^{1}\right|^{2}+\tilde{a}_{t}^{2}\left|b_{t, *}^{2}\right|^{2}+2 \sqrt{\tilde{a}_{t}^{1} \tilde{a}_{t}^{2} \operatorname{det}\left(b_{t, *} b_{t, *}^{\top}\right)}\right)^{1 / 2}}{2}\right\} d t .
\end{aligned}
$$

Proposition 2.1. Assume (A1), (A2) and (V). Then $\sup _{\sigma \in \Lambda} \mid b_{n}^{-1 / 2} \partial_{\sigma}^{k}\left(H_{n}\left(\sigma, \hat{v}_{n}\right)-H_{n}\left(\sigma_{*}, \hat{v}_{n}\right)\right)-$ $\partial_{\sigma}^{k} \mathcal{Y}_{1}(\sigma) \mid \rightarrow^{p} 0$ as $n \rightarrow \infty$ for $0 \leq k \leq 3$. 
To show consistency and asymptotic normality of $\hat{\sigma}_{n}$, the limit function $\mathcal{Y}_{1}(\sigma)$ of the quasilikelihood ratio should have the unique maximum point at $\sigma=\sigma_{*}$. More precisely, we use the following as a kind of identifiability condition: $\inf _{\sigma \neq \sigma_{*}}\left(-\mathcal{Y}_{1}(\sigma)\right) /\left|\sigma-\sigma_{*}\right|^{2}>0$ almost surely. Though it is difficult to directly check this condition in general, we can check it under a more tractable sufficient condition. Let

$$
\mathcal{Y}_{0}(\sigma)=-\frac{1}{2} \int_{0}^{T}\left\{\operatorname{tr}\left(\left(b_{t} b_{t}^{\top}\right)^{-1}\left(b_{t, *} b_{t, *}^{\top}\right)-\mathcal{E}_{2}\right)+\log \frac{\operatorname{det}\left(b_{t} b_{t}^{\top}\right)}{\operatorname{det}\left(b_{t, *} b_{t, *}^{\top}\right)}\right\} d t
$$

Then $\mathcal{Y}_{0}$ is the probability limit $n^{-1 / 2}\left(H_{n}^{0}(\sigma)-H_{n}^{0}\left(\sigma_{*}\right)\right)$, where $H_{n}^{0}$ represents a quasi-likelihood function for a statistical model of equidistant observations without noise. See Uchida and Yoshida [28].

Assumption (A3). $\inf _{\sigma \neq \sigma_{*}}\left(\left(-\mathcal{Y}_{0}(\sigma)\right) /\left|\sigma-\sigma_{*}\right|^{2}\right)>0$ almost surely.

We will show in Proposition 6.1 that (A3) is sufficient for the identifiability condition of our model. Moreover, the following condition is a simple sufficient condition for (A3) (see Remark 4 in Ogihara and Yoshida [25] for the details).

Assumption $\left(\mathbf{A 3}^{\prime}\right) . \inf _{\sigma_{1} \neq \sigma_{2}}\left(\left|b b^{\top}\left(t, x, \sigma_{1}\right)-b b^{\top}\left(t, x, \sigma_{2}\right)\right| /\left|\sigma_{1}-\sigma_{2}\right|\right)>0$ for any $t \in[0, T]$ and $x \in O$.

We denote by $\rightarrow^{s-\mathcal{L}}$ the stable convergence of random variables. Let

$$
\hat{\Gamma}_{1, n}=-b_{n}^{-1 / 2} \partial_{\sigma}^{2} H_{n}\left(\hat{\sigma}_{n}, \hat{v}_{n}\right), \quad \Gamma_{1}=-\partial_{\sigma}^{2} \mathcal{Y}_{1}\left(\sigma_{*}\right)
$$

Let $\mathcal{N}$ be a $d$-dimensional random variable on some extension $(\tilde{\Omega}, \tilde{\mathcal{F}}, \tilde{P})$ of $(\Omega, \mathcal{F}, P)$ satisfying the condition that $\mathcal{N}$ is independent of $\mathcal{F}$ and $\mathcal{N}$ follows the $d$-dimensional standard normal distribution. We denote the expectation with respect to $\tilde{P}$ by the same notation $E$.

The following theorem is one of our main results.

Theorem 2.1. Assume (A1)-(A3) and (V). Then $\Gamma_{1}$ is positive definite almost surely and $b_{n}^{1 / 4}\left(\hat{\sigma}_{n}-\sigma_{*}\right) \rightarrow^{s-\mathcal{L}} \Gamma_{1}^{-1 / 2} \mathcal{N}$ as $n \rightarrow \infty$. Moreover, $\hat{\Gamma}_{1, n} \rightarrow^{p} \Gamma_{1}$, and therefore $b_{n}^{1 / 4} \times$ $\hat{\Gamma}_{1, n}^{1 / 2} 1_{\left\{\hat{\Gamma}_{1, n} \text { is p.d. }\right\}}\left(\hat{\sigma}_{n}-\sigma_{*}\right) \rightarrow^{s-\mathcal{L}} \mathcal{N}$ as $n \rightarrow \infty$.

Corollary 2.1. Assume (A1), (A2), (A3') and (V). Then the results in Theorem 2.1 hold true.

Let $\Upsilon_{t}^{(k)}=\left.\partial_{\sigma}^{k}\left(\tilde{a}_{t}^{1}\left|b_{t}^{1}\right|^{2}+\tilde{a}_{t}^{2}\left|b_{t}^{2}\right|^{2}+2 \sqrt{\tilde{a}_{t}^{1} \tilde{a}_{t}^{2} \operatorname{det}\left(b_{t} b_{t}^{\top}\right)}\right)^{1 / 2}\right|_{\sigma=\sigma_{*}}, B_{j, t}^{(k)}=\left.\partial_{\sigma}^{k}\left|b_{t}^{j}\right|^{2}\right|_{\sigma=\sigma_{*}}, B_{3, t}^{(k)}=$ $\left.\partial_{\sigma}^{k} b_{t}^{1} \cdot b_{t}^{2}\right|_{\sigma=\sigma_{*}}$, and $B_{4, t}^{(k)}=\left.\partial_{\sigma}^{k} \sqrt{\operatorname{det}\left(b_{t} b_{t}^{\top}\right)}\right|_{\sigma=\sigma_{*}}$ for $k=0,1$ and $j=1,2$. Then we can rewrite 
$\Gamma_{1}$ as

$$
\begin{aligned}
\Gamma_{1}= & \int_{0}^{T}\left\{\frac{\Upsilon_{t}^{(1)}\left(\Upsilon_{t}^{(1)}\right)^{\top}}{2 \Upsilon_{t}^{(0)}}+\frac{\sqrt{\tilde{a}_{t}^{1} \tilde{a}_{t}^{2}}}{2 B_{4, t}^{(0)} \Upsilon_{t}^{(0)}}\left(B_{4, t}^{(1)}\left(B_{4, t}^{(1)}\right)^{\top}\right.\right. \\
& \left.\left.-\frac{B_{1, t}^{(1)}\left(B_{2, t}^{(1)}\right)^{\top}+B_{2, t}^{(1)}\left(B_{1, t}^{(1)}\right)^{\top}}{2}+B_{3, t}^{(1)}\left(B_{3, t}^{(1)}\right)^{\top}\right)\right\} d t .
\end{aligned}
$$

The derivation of (2.10) is left to the appendix.

\subsection{On the LAMN property and asymptotic efficiency of the estimator}

In this subsection, we state some results on the so-called LAMN (LAN) property for our model and asymptotic efficiency of our estimator. We also comment on some further studies.

Throughout this subsection, we assume that $X_{t} \equiv Y_{t}, T_{j}^{n, k} \equiv S_{i}^{n, k}, \eta_{j}^{n, k} \equiv \varepsilon_{i}^{n, k}, \mu_{t}=\mu\left(t, \sigma_{*}\right)$ and $Y_{0}=\gamma$ for some Borel function $\mu$ and some known $\gamma \in \mathbb{R}^{2}$. Then the latent process $Y$ is a diffusion process satisfying the stochastic differential equation (2.3) with $\mu=\mu\left(t, \sigma_{*}\right)$. Let $P_{\sigma_{*}^{\prime}, v_{*}^{\prime}, n}$ be the distribution of $\left(\left(S_{i}^{n, k}\right)_{k, i},\left(\tilde{Y}_{i}^{k}\right)_{k, i}\right)$ with true values $\left(\sigma_{*}^{\prime}, v_{*}^{\prime}\right)$ of the parameters. We denote

$$
\operatorname{diag}(A, B)=\left(\begin{array}{cc}
A & 0 \\
0 & B
\end{array}\right)
$$

for square matrices $A$ and $B$. Let $\mathcal{Y}_{2}(v)=-\int_{0}^{T} \sum_{j=1}^{2} a_{t}^{j}\left\{\left(v_{j, *} / v_{j}\right)-1+\log \left(v_{j} / v_{j, *}\right)\right\} d t / 2$,

$$
\Gamma_{2}=-\partial_{v}^{2} \mathcal{Y}_{2}\left(v_{*}\right) \quad \text { and } \quad \Gamma=\operatorname{diag}\left(\Gamma_{1}, \Gamma_{2}\right) .
$$

We adopt the following definition of the LAMN property from Jeganathan [21].

Definition 2.1. Let $P_{\theta, n}$ be a probability measure on some measurable space $\left(\mathcal{X}_{n}, \mathcal{A}_{n}\right)$ for each $\theta \in \boldsymbol{\Theta}$ and $n \in \mathbb{N}$, where $\Theta$ is a bounded open subset of $\mathbb{R}^{d}$. Then the family $\left\{P_{\theta, n}\right\}_{\theta, n}$ satisfies the local asymptotic mixed normality (LAMN) property at $\theta=\theta_{*}$ if there exist a sequence $\left\{\delta_{n}\right\}_{n \in \mathbb{N}}$ of $d \times d$ positive definite matrices, $d \times d$ symmetric random matrices $\Gamma_{n}, \Gamma$ and $d$-dimensional random vectors $\mathcal{N}_{n}, \mathcal{N}$ such that $\Gamma$ is positive definite a.s., $P_{\theta_{*}, n}\left[\Gamma_{n}\right.$ is positive definite $]=1$ ( $n \in$ $\mathbb{N}),\left\|\delta_{n}\right\| \rightarrow 0$, and

$$
\log \frac{d P_{\theta_{*}+\delta_{n} u, n}}{d P_{\theta_{*}, n}}-\left(u^{\top} \sqrt{\Gamma_{n}} \mathcal{N}_{n}-\frac{1}{2} u^{\top} \Gamma_{n} u\right) \rightarrow 0
$$

in $P_{\theta_{*}, n}$-probability as $n \rightarrow \infty$ for any $u \in \mathbb{R}^{d}$. Moreover, $\mathcal{N}$ follows the $d$-dimensional standard normal distribution, $\mathcal{N}$ is independent of $\Gamma$ and $\mathcal{L}\left(\mathcal{N}_{n}, \Gamma_{n} \mid P_{\theta_{*}, n}\right) \rightarrow \mathcal{L}(\mathcal{N}, \Gamma)$ as $n \rightarrow \infty$.

If further the limit matrix $\Gamma$ is non-random, we say $\left\{P_{\theta, n}\right\}_{\theta, n}$ has the local asymptotic normality (LAN) property. 
To prove the LAMN property of our model, we assume the following additional condition.

Assumption (A1"). (A1) is satisfied, $\mu_{t}=\mu\left(t, \sigma_{*}\right), \sup _{t, \sigma} \mu(t, \sigma)<\infty, b(t, x, \sigma)$ does not depend on $x$ and $\varepsilon_{i}^{n, k}$ follows a normal distribution for any $n, k, i$.

Theorem 2.2. Assume (A1"), (A2) and (A3). Then the family of distributions $\left\{P_{\sigma_{*}, v_{*}, n}\right\}_{\sigma_{*}, v_{*}, n}$ has the LAN property with $\Gamma$ in (2.11) and $\delta_{n}=\operatorname{diag}\left(b_{n}^{-1 / 4} \mathcal{E}_{d}, b_{n}^{-1 / 2} \mathcal{E}_{2}\right)$.

Remark 2.6. Jeganathan [20] studied lower bounds of estimation errors for any estimator of parameters. They showed a version of Hájek's convolution theorem (Corollary 1) and that the optimal asymptotic variance of errors for regular estimators is $\Gamma^{-1}$, where $\Gamma$ is in Definition 2.1. Therefore, Theorems 2.1 and 2.2 ensures that our estimator $\hat{\sigma}_{n}$ of the parameter $\sigma$ is asymptotically efficient in this sense under the assumptions of both theorems.

Remark 2.7. The assumptions of Theorem 2.2 are rather strong conditions. We are also interested in the LAMN property in more general settings. In particular, we are interested in the case that $\mu_{t}=\mu\left(t, X_{t}, \sigma_{*}\right)$ and $\mu$ and $b$ are general functions with suitable conditions. However, we need further analysis using Malliavin calculus to deal with the LAMN property of general diffusion processes, as seen in Gobet [14] and Ogihara [24]. Moreover, when we deal with nonsynchronousness or noise, the true likelihood function is obtained by integrating a likelihood function of ideal observations with respect to unobservable variables. Such integral is not easy to handle, and consequently, it makes the proof of LAMN much more complicated as seen in [24]. To the best of author's knowledge, the LAMN property for a general diffusion model has not been shown even for models with noisy, synchronous observations. We have left this for future works. On the other hand, asymptotic results of the quasi-likelihood function shown in this paper (e.g., Propositions 2.1 and 7.2) are expected to be useful when we try to show LAMN for general cases. Indeed, LAMN for a model with nonsynchronous observations is proved in [24] by showing asymptotic equivalence of the true likelihood ratio and a quasi-likelihood ratio, and using asymptotic results of the quasi-likelihood ratio in Ogihara and Yoshida [25].

\subsection{A Bayes-type estimator and convergence of moments of estimation errors}

Polynomial-type large deviation theory by Yoshida [29,30] enables us to address the asymptotic properties of a Bayes-type estimator and the convergence of moments of estimation errors, which is a stronger result than asymptotic mixed normality. Convergence of moments is useful when we investigate the theory of information criteria, minimax inequality and asymptotic expansion of estimators. See Uchida [27] for a theory of contrast-based information criteria for ergodic diffusion processes with equidistant observations. We also see asymptotic efficiency of our estimator in the sense of minimax inequality.

We first assume following stronger conditions than (A1)-(A3) and (V). 
Assumption (B1). 1. (A1) holds true with $O=\mathbb{R}^{d_{2}}$.

2. There exists a positive constant $C$ such that $\sup _{t \in[0, T], \sigma \in \Lambda}\left|\partial_{t}^{i} \partial_{x}^{j} \partial_{\sigma}^{k} b(t, x, \sigma)\right| \leq C(1+|x|)^{C}$ for $0 \leq 2 i+j \leq 4,0 \leq k \leq 4$ and $x \in \mathbb{R}^{d_{2}}$.

3. $\inf _{t, x, \sigma} \operatorname{det} b b^{\top}(t, x, \sigma)>0$.

4. $E\left[\left|Y_{0}\right|^{q}\right]<\infty$ for any $q>0$.

5. $\sup _{t} E\left[\left|\mu_{t}\right|^{q}\right]<\infty, \sup _{s<t}\left(E\left[\left|\mu_{t}-\mu_{s}\right|^{q}\right]^{1 / q}(t-s)^{-1 / 2}\right)<\infty$ and $\sup _{s<t} E\left[\left(E\left[\mu_{t}-\right.\right.\right.$ $\left.\left.\left.\mu_{s} \mid \mathcal{G}_{s}\right] /(t-s)\right)^{q}\right]<\infty$ for any $q>0$.

6. For any $q>0, \max _{j} \sup _{t} E\left[\left|b_{t}^{(j)}\right|^{q} \vee\left|\hat{b}_{t}^{(j)}\right|^{q}\right]<\infty$ and $\max _{j} \sup _{s<t}\left(E\left[\left|b_{t}^{(j)}-b_{s}^{(j)}\right|^{q} \vee\right.\right.$ $\left.\left.\left|\hat{b}_{t}^{(j)}-\hat{b}_{s}^{(j)}\right|^{q}\right]^{1 / q}(t-s)^{-1 / 2}\right)<\infty$.

Assumption (B2). There exist $\eta \in(0,1 / 2), \dot{\eta} \in(0,1], \delta>0$ and positive-valued stochastic processes $\left\{a_{t}^{j}\right\}_{t \in[0, T], j=1,2}$ such that $b_{n}^{-1 / 2} k_{n}\left(b_{n}^{-1} k_{n}\right)^{\dot{\eta}} \rightarrow 0$ as $n \rightarrow \infty, E\left[\sup _{j, t>s}\left(\left|a_{t}^{j}-a_{s}^{j}\right|^{q} \mid t-\right.\right.$ $\left.\left.\left.s\right|^{-q \dot{\eta}}\right)\right]<\infty, E\left[\sup _{j, t}\left|a_{t}^{j}\right|^{q}\right] \vee E\left[\sup _{j, t}\left(\left|a_{t}^{j}\right|^{-q}\right)\right]<\infty$, and

$$
\begin{aligned}
& \sup _{n} \sup _{\left\{\left[s_{n, l}^{\prime}, s_{n, l}^{\prime \prime}\right)\right\} \in \mathcal{S}_{\eta}} E\left[\left(k_{n} b_{n}^{-1 / 2+\delta} \max _{1 \leq l \leq L_{n}} \mid b_{n}^{-1}\left(s_{n, l}^{\prime \prime}-s_{n, l}^{\prime}\right)^{-1} \#\left\{i ;\left[S_{i-1}^{n, j}, S_{i}^{n, j}\right)\right.\right.\right. \\
& \left.\left.\left.\quad \subset\left[s_{n, l}^{\prime}, s_{n, l}^{\prime \prime}\right)\right\}-a_{s_{n, l}^{\prime}}^{j} \mid\right)^{q}\right]
\end{aligned}
$$

is finite for any $q>0$. Moreover, there exists a positive constant $\gamma$ such that $k_{n} b_{n}^{-4 / 7+\gamma} \rightarrow 0$ and $E\left[\left(\left(r_{n} b_{n}^{1-\varepsilon}\right) \vee\left(\underline{\mathrm{r}}_{n}^{-1} b_{n}^{-1-\varepsilon}\right)\right)^{q}\right] \rightarrow 0$ as $n \rightarrow \infty$ for any $q>0$ and $\varepsilon>0$.

Assumption (B3). For any $q>0$, there exists a positive constant $c_{q}$ such that $P\left[\inf _{\sigma \neq \sigma_{*}}\left(\left(-\mathcal{Y}_{0}(\sigma)\right) /\left|\sigma-\sigma_{*}\right|^{2}\right) \leq r^{-1}\right] \leq c_{q} / r^{q}$ for any $r>0$.

Assumption (B4). There exist estimators $\left\{\hat{v}_{n}\right\}_{n \in \mathbb{N}}$ of $v_{*}$ such that $\hat{v}_{n}>0$ almost surely, $\limsup \sup _{n} E\left[\hat{v}_{n}^{-q}\right]<\infty, \sup _{n} E\left[\left|b_{n}^{1 / 2}\left(\hat{v}_{n}-v_{*}\right)\right|^{q}\right]<\infty$, and $\sup _{n} E\left[b_{n}^{-\varepsilon} \max _{m, k}\left(E_{\Pi}\left[\mid \ell_{n}^{1 / 2} \times\right.\right.\right.$ $\left.\left.\left.\left.\mathbf{T}_{m, k}\right|^{q} \vee\left|\ell_{n} E\left[\mathbf{T}_{m, k} \mid \mathcal{G}_{s_{m-1}}\right]\right|^{q}\right]\right)\right]<\infty$ for any $q>0$ and $\varepsilon>0$.

Though Assumption (B3) is rather difficult to check in a practical setting, Uchida and Yoshida [28] investigated sufficient conditions for (B3). The simplest condition is that (B3) is satisfied if there exists $\varepsilon>0$ such that $\left|b b^{\top}\left(t, x, \sigma_{1}\right)-b b^{\top}\left(t, x, \sigma_{2}\right)\right| \geq \varepsilon\left|\sigma_{1}-\sigma_{2}\right|$ for any $t \in[0, T], x \in O$ and $\sigma_{1}, \sigma_{2} \in \Lambda$. See Remark 4 in [25] for details.

Let $U_{n}=\left\{u \in \mathbb{R}^{d} ; \sigma_{*}+b_{n}^{-1 / 4} u \in \Lambda\right\}, V_{n}(r)=\{|u| \geq r\} \cap U_{n}$, and $\mathbf{Z}_{n}(u)=\exp \left(H_{n}\left(\sigma_{*}+\right.\right.$ $\left.\left.b_{n}^{-1 / 4} u, \hat{v}_{n}\right)-H_{n}\left(\sigma_{*}, \hat{v}_{n}\right)\right)$ for $u \in U_{n}$.

Proposition 2.2 (Polynomial-type large deviation inequalities). Assume (B1)-(B4). Then for any $L>0$, there exists a positive constant $c_{L}$ such that $P\left[\sup _{u \in V_{n}(r)} \mathbf{Z}_{n}(u) \geq e^{-r / 2}\right] \leq c_{L} / r^{L}$ for any $n \in \mathbb{N}$ and $r>0$. 
Since $\mathbf{Z}_{n}(0)=1$, Proposition 2.2 immediately yields

$$
\begin{aligned}
E\left[\left|b_{n}^{1 / 4}\left(\hat{\sigma}_{n}-\sigma_{*}\right)\right|^{p}\right] & =\int_{0}^{\infty} p t^{p-1} P\left[\left|b_{n}^{1 / 4}\left(\hat{\sigma}_{n}-\sigma_{*}\right)\right| \geq t\right] d t \\
& \leq \int_{0}^{\infty} p t^{p-1} P\left[\sup _{u \in V_{n}(t)} \mathbf{Z}_{n}(u) \geq e^{-t / 2}\right] d t<\infty
\end{aligned}
$$

for any $p>0$. Moreover, we obtain the following convergence of moments of the estimation error.

Theorem 2.3. Assume (B1)-(B4). Then $E\left[\mathbf{Y} f\left(b_{n}^{1 / 4}\left(\hat{\sigma}_{n}-\sigma_{*}\right)\right)\right] \rightarrow E\left[\mathbf{Y} f\left(\Gamma_{1}^{-1 / 2} \mathcal{N}\right)\right]$ as $n \rightarrow$ $\infty$ for any bounded random variable $\mathbf{Y}$ on $(\Omega, \mathcal{F})$ and any continuous function $f$ of at most polynomial growth.

In particular, we obtain convergence of moments where $E\left[\left|b_{n}^{1 / 4}\left(\hat{\sigma}_{n}-\sigma_{*}\right)\right|^{q}\right] \rightarrow E\left[\left|\Gamma_{1}^{-1 / 2} \mathcal{N}\right|^{q}\right]$ for any $q>0$. This property is used when we study the theory of information criteria and asymptotic expansion of estimators.

We also obtain results for a Bayes type estimator. Let a prior density $\pi: \Lambda \rightarrow(0, \infty)$ be a continuous function satisfying $0<\inf _{\sigma} \pi(\sigma) \leq \sup _{\sigma} \pi(\sigma)<\infty$. Then a Bayes-type estimator $\tilde{\sigma}_{n}$ for the quadratic loss function is defined by

$$
\tilde{\sigma}_{n}=\left(\int_{\Lambda} \exp \left(H_{n}\left(\sigma, \hat{v}_{n}\right)\right) \pi(\sigma) d \sigma\right)^{-1} \int_{\Lambda} \sigma \exp \left(H_{n}\left(\sigma, \hat{v}_{n}\right)\right) \pi(\sigma) d \sigma .
$$

Since the Bayes-type estimator $\tilde{\sigma}_{n}$ contains integrals with respect to $\sigma$, we need to deal with tail behaviors of likelihood ratio $H_{n}\left(\sigma, \hat{v}_{n}\right)-H_{n}\left(\sigma_{*}, \hat{v}_{n}\right)$. Hence, Proposition 2.2 is essential to deduce asymptotic properties of a Bayes-type estimator. Since the Bayes-type estimator can be calculated using Markov-Chain Monte Carlo methods, it is often easier to calculate than the maximum-likelihood-type estimator. For the Bayes-type estimator $\tilde{\sigma}_{n}$, we obtain similar results to the ones for the maximum-likelihood-type estimator.

Theorem 2.4. Assume (B1)-(B4). Then $E\left[\mathbf{Y} f\left(b_{n}^{1 / 4}\left(\tilde{\sigma}_{n}-\sigma_{*}\right)\right)\right] \rightarrow E\left[\mathbf{Y} f\left(\Gamma_{1}^{-1 / 2} \mathcal{N}\right)\right]$ as $n \rightarrow$ $\infty$ for any bounded random variable $\mathbf{Y}$ on $(\Omega, \mathcal{F})$ and any continuous function $f$ of at most polynomial growth.

Remark 2.8. If the assumptions of Theorem 2.2 are satisfied, the asymptotic minimax theorem (Theorem 4 in [21]) holds for our model, so

$$
\lim _{\alpha \rightarrow \infty} \liminf _{n \rightarrow \infty} \sup _{|u| \leq \alpha} E_{\sigma_{*}+b_{n}^{-1 / 4} u}\left[l\left(\left|b_{n}^{1 / 4}\left(V_{n}-\sigma_{*}-b_{n}^{-1 / 4} u\right)\right|\right)\right] \geq E\left[l\left(\left|\Gamma_{1} \mathcal{N}\right|\right)\right]
$$

for any estimators $\left\{V_{n}\right\}_{n}$ of the parameter and any function $l:[0, \infty) \rightarrow[0, \infty)$ which is nondecreasing and $l(0)=0$, where $E_{\sigma}$ denotes expectation with respect to $P_{\sigma, v_{*}, n}$. Using Theorems 2.3 and 2.4 and a similar argument in Theorem 2.2 of Ogihara [24], we can see that $\hat{\sigma}_{n}$ and $\tilde{\sigma}_{n}$ attain 
the lower bound of the above inequality for continuous $l$ of at most polynomial growth, if further (B2) and uniform versions of (B3) and (B4) with respect to the true value $\left(\sigma_{*}, v_{*}\right)$ are satisfied. Hence our estimators are asymptotically efficient in this sense as well.

\section{Simulation results}

In this section, we examine some simulation results of our estimator.

First, we consider the case where the latent process $Y$ is a Brownian motion, that is, $Y$ satisfies the following stochastic differential equation:

$$
\left\{\begin{array}{l}
d Y_{t}^{1}=\sigma_{1, *} d W_{t}^{1}, \\
d Y_{t}^{2}=\sigma_{3, *} d W_{t}^{1}+\sigma_{2, *} d W_{t}^{2},
\end{array}\right.
$$

where $\sigma_{*}=\left(\sigma_{1, *}, \sigma_{2, *}, \sigma_{3, *}\right) \in(\varepsilon, R) \times(-R, R) \times(\varepsilon, R)$ for some $0<\varepsilon<R$. Moreover, let $\left\{N_{t}^{1}\right\}_{0 \leq t \leq T}$ and $\left\{N_{t}^{2}\right\}_{0 \leq t \leq T}$ be two independent Poisson processes with parameters $\lambda_{1}$ and $\lambda_{2}$, respectively. We give sampling times by $S_{i}^{n, j}=\inf \left\{N_{n t}^{j} \geq j\right\} \wedge T$ for $j=1,2$. Let $\left\{\varepsilon_{i}^{n, j}\right\}_{i \in \mathbb{Z}_{+}, j=1,2}$ be independent normal random variables with $E\left[\varepsilon_{i}^{n, j}\right]=0$ and $E\left[\left(\varepsilon_{i}^{n, j}\right)^{2}\right]=v_{j, *}$.

Then we can see that this example satisfies (A1"), (A2) and $\left(\mathrm{A}^{\prime}\right)$. So the maximumlikelihood-type estimator $\hat{\sigma}_{n}$ is asymptotically mixed normal and asymptotically efficient with the asymptotic variance $\Gamma_{1}^{-1}$. For the estimator $\hat{v}_{n}$ of $v_{*}$ we first use a simple estimator $\hat{v}_{n}=\left(2 \mathbf{J}_{k, n}\right)^{-1} \sum_{i}\left(\tilde{Y}_{i}^{k}-\tilde{Y}_{i-1}^{k}\right)^{2}$, which means that our estimator is calculated by $\hat{\sigma}_{n}=$ $\operatorname{argmax}_{\sigma} H_{n}\left(\sigma, \hat{v}_{n}\right)$. We also consider a plug-in estimator $\hat{v}_{k, n}^{\prime}=\left(\hat{v}_{k, n}-\left|b^{k}\left(\hat{\sigma}_{n}\right)\right|^{2} T /\left(2 \mathbf{J}_{k, n}\right)\right) \vee 0$ of $v_{k, *}$, and $\hat{\sigma}_{n}^{\prime}=\operatorname{argmax}_{\sigma} H_{n}\left(\sigma, \hat{v}_{n}^{\prime}\right)$. Let $\hat{\sigma}_{n}^{\prime \prime}=\operatorname{argmax}_{\sigma} H_{n}\left(\sigma, v_{*}\right)$. Then $\hat{\sigma}_{n}^{\prime \prime}$ cannot be calculated by observed data, but we can use it for comparison. Though these estimators have the same asymptotic variance, their performances for finite samples are different. In particular, we cannot ignore the bias of $\hat{v}_{n}$ since $v$ is relatively small compared with $\sigma$ in practical data.

Table 1 shows results of 1000 independent estimations. Each cell represents the average of estimators, with sample standard deviations given in parentheses. We set the values of parameters as $k_{n}=\left[n^{5 / 8}\right], T=1,\left(\lambda_{1}, \lambda_{2}\right)=(1,1),\left(\sigma_{1, *}, \sigma_{2, *}, \sigma_{3, *}\right)=\left(1, \sqrt{1-0.5^{2}}, 0.5\right)$, and consider two cases of the noise variances : $v_{*}=(0.001,0.001)$ and $v_{*}=(0.005,0.005)$. In both cases, we can see that $\hat{v}_{n}$ has an upper bias for $n=1000$, and causes a lower bias of $\hat{\sigma}_{n}$ because $\hat{v}_{n}$ contains variance of the latent process, which is always positive. These biases can be moderated by using the plug-in estimator. For $n=5000$, the plug-in estimator $\hat{\sigma}_{n}^{\prime}$ performs as well as $\hat{\sigma}_{n}^{\prime \prime}$. In the case of $v_{*}=(0.005,0.005)$, the biases of $\hat{v}_{n}$ and $\hat{v}_{n}^{\prime}$ are relatively small, so the performance of $\hat{\sigma}_{n}$ and $\hat{\sigma}_{n}^{\prime}$ are better.

We can also construct an estimator $\hat{\sigma}_{1, n}^{\prime} \hat{\sigma}_{3, n}^{\prime} T$ of the quadratic covariation $\left\langle Y^{1}, Y^{2}\right\rangle_{T}=$ $\sigma_{1, *} \sigma_{3, *} T$. We see that

$$
\begin{aligned}
& n^{1 / 4}\left(\hat{\sigma}_{1, n}^{\prime} \hat{\sigma}_{3, n}^{\prime} T-\left\langle Y^{1}, Y^{2}\right\rangle_{T}\right) \\
& \quad \stackrel{d}{\rightarrow} N\left(0, T^{2}\left(\sigma_{3, *}^{2}\left(\Gamma_{1}^{-1}\right)_{11}+2 \sigma_{1, *} \sigma_{3, *}\left(\Gamma_{1}^{-1}\right)_{13}+\sigma_{1, *}^{2}\left(\Gamma_{1}^{-1}\right)_{33}\right)\right)
\end{aligned}
$$


Table 1. Simulation results for estimators of parameters

\begin{tabular}{|c|c|c|c|c|c|c|}
\hline \multicolumn{7}{|c|}{ Results with $v_{*}=(0.001,0.001)$} \\
\hline \multicolumn{2}{|l|}{$n$} & \multirow{2}{*}{$\begin{array}{l}\sigma_{1} \\
0.897 \\
(0.040)\end{array}$} & \multirow{2}{*}{$\begin{array}{l}\sigma_{2} \\
0.776 \\
(0.042)\end{array}$} & \multirow{2}{*}{$\begin{array}{l}\sigma_{3} \\
0.451 \\
(0.062)\end{array}$} & \multirow{2}{*}{$\begin{array}{l}v_{1} \\
0.001504 \\
(0.000079)\end{array}$} & \multirow{2}{*}{$\begin{array}{l}v_{2} \\
0.001500 \\
(0.000080)\end{array}$} \\
\hline 1000 & $\left(\hat{\sigma}_{n}, \hat{v}_{n}\right)$ & & & & & \\
\hline & $\left(\hat{\sigma}_{n}^{\prime}, \hat{v}_{n}^{\prime}\right)$ & $\begin{array}{l}0.971 \\
(0.046)\end{array}$ & $\begin{array}{l}0.840 \\
(0.047)\end{array}$ & $\begin{array}{l}0.487 \\
(0.067)\end{array}$ & $\begin{array}{l}0.001100 \\
(0.000075)\end{array}$ & $\begin{array}{l}0.001094 \\
(0.000078)\end{array}$ \\
\hline & $\hat{\sigma}_{n}^{\prime \prime}$ & 0.999 & 0.863 & 0.501 & - & - \\
\hline & & $(0.045)$ & $(0.046)$ & $(0.068)$ & - & - \\
\hline \multirow[t]{6}{*}{5000} & $\left(\hat{\sigma}_{n}, \hat{v}_{n}\right)$ & 0.964 & 0.833 & 0.481 & 0.001099 & 0.001099 \\
\hline & & $(0.028)$ & $(0.029)$ & $(0.040)$ & $(0.000026)$ & $(0.000026)$ \\
\hline & $\left(\hat{\sigma}_{n}^{\prime}, \hat{v}_{n}^{\prime}\right)$ & 0.997 & 0.862 & 0.498 & 0.001006 & 0.001006 \\
\hline & & $(0.031)$ & $(0.031)$ & $(0.041)$ & $(0.000027)$ & $(0.000027)$ \\
\hline & $\hat{\sigma}_{n}^{\prime \prime}$ & 0.999 & 0.864 & 0.499 & - & - \\
\hline & & $(0.029)$ & $(0.030)$ & $(0.041)$ & - & - \\
\hline \multicolumn{2}{|c|}{ True values } & 1 & 0.866 & 0.5 & 0.001 & 0.001 \\
\hline \multicolumn{7}{|c|}{ Results with $v_{*}=(0.005,0.005)$} \\
\hline \multicolumn{2}{|l|}{$n$} & $\sigma_{1}$ & $\sigma_{2}$ & $\sigma_{3}$ & $v_{1}$ & $v_{2}$ \\
\hline \multirow[t]{5}{*}{1000} & $\left(\hat{\sigma}_{n}, \hat{v}_{n}\right)$ & $\begin{array}{l}0.957 \\
(0.086)\end{array}$ & $\begin{array}{l}0.818 \\
(0.143)\end{array}$ & $\begin{array}{l}0.481 \\
(0.094)\end{array}$ & $\begin{array}{l}0.005515 \\
(0.000293)\end{array}$ & $\begin{array}{l}0.005501 \\
(0.000296)\end{array}$ \\
\hline & $\left(\hat{\sigma}_{n}^{\prime}, \hat{v}_{n}^{\prime}\right)$ & 0.991 & 0.850 & 0.498 & 0.005053 & 0.005035 \\
\hline & & $(0.092)$ & $(0.139)$ & $(0.098)$ & $(0.000298)$ & $(0.000306)$ \\
\hline & $\hat{\sigma}_{n}^{\prime \prime}$ & 0.997 & 0.861 & 0.499 & - & - \\
\hline & & $(0.069)$ & $(0.070)$ & $(0.096)$ & - & - \\
\hline \multirow[t]{6}{*}{5000} & $\left(\hat{\sigma}_{n}, \hat{v}_{n}\right)$ & 0.990 & 0.854 & 0.495 & 0.005095 & 0.005096 \\
\hline & & $(0.044)$ & $(0.044)$ & $(0.061)$ & $(0.000121)$ & $(0.000123)$ \\
\hline & $\left(\hat{\sigma}_{n}^{\prime}, \hat{v}_{n}^{\prime}\right)$ & 0.999 & 0.862 & 0.499 & 0.004996 & 0.004998 \\
\hline & & $(0.045)$ & $(0.045)$ & $(0.062)$ & $(0.000123)$ & $(0.000125)$ \\
\hline & $\hat{\sigma}_{n}^{\prime \prime}$ & 0.998 & 0.862 & 0.499 & - & - \\
\hline & & $(0.043)$ & $(0.044)$ & $(0.062)$ & - & - \\
\hline \multicolumn{2}{|c|}{ True values } & 1 & 0.866 & 0.5 & 0.005 & 0.005 \\
\hline
\end{tabular}

as $n \rightarrow \infty$ by the delta method, and the estimator is asymptotically efficient since we can reparameterize the model using $\sigma_{1, *} \sigma_{3, *}$. We therefore compared the performance of the estimator (MLE) with existing estimators of the quadratic covariation. We used the pre-averaged Hayashi-Yoshida estimator (PHY) and modulated realised covariance (MRC) by Christensen, Kinnebrock, and Podolskij [7], the local method of moments (LMM) by Bibinger et al. [5], and an estimator based on maximum likelihood estimator of a model of constant diffusion coefficients (QMLE) by Aït-Sahalia, Fan, and Xiu [2] for comparison. Except LMM these estimators 
Table 2. Comparison of estimators of $\left\langle Y^{1}, Y^{2}\right\rangle_{T}$

\begin{tabular}{|c|c|c|c|c|c|c|c|}
\hline \multicolumn{8}{|c|}{ Resuls with $v_{*}=(0.001,0.001)$} \\
\hline$n$ & MLE & PHY & $\mathrm{MRC}_{1}$ & $\mathrm{MRC}_{2}$ & QMLE & LMM & minimum \\
\hline 1000 & $\begin{array}{l}0.474 \\
(0.073)\end{array}$ & $\begin{array}{l}0.499 \\
(0.121)\end{array}$ & $\begin{array}{l}0.508 \\
(0.182)\end{array}$ & $\begin{array}{l}0.501 \\
(0.110)\end{array}$ & $\begin{array}{l}0.501 \\
(0.095)\end{array}$ & $\begin{array}{l}0.463 \\
(0.082)\end{array}$ & $(0.066)$ \\
\hline 5000 & $\begin{array}{l}0.496 \\
(0.046)\end{array}$ & $\begin{array}{l}0.497 \\
(0.081)\end{array}$ & $\begin{array}{l}0.504 \\
(0.124)\end{array}$ & $\begin{array}{l}0.499 \\
(0.073)\end{array}$ & $\begin{array}{l}0.498 \\
(0.056)\end{array}$ & $\begin{array}{l}0.497 \\
(0.069)\end{array}$ & $(0.044)$ \\
\hline \multicolumn{8}{|c|}{ Results with $v_{*}=(0.005,0.005)$} \\
\hline$n$ & MLE & PHY & $\mathrm{MRC}_{1}$ & $\mathrm{MRC}_{2}$ & QMLE & LMM & minimum \\
\hline 1000 & $\begin{array}{l}0.496 \\
(0.109)\end{array}$ & $\begin{array}{l}0.497 \\
(0.148)\end{array}$ & $\begin{array}{l}0.508 \\
(0.185)\end{array}$ & $\begin{array}{l}0.5000 \\
(0.124)\end{array}$ & $\begin{array}{l}0.5000 \\
(0.120)\end{array}$ & $\begin{array}{l}0.518 \\
(0.112)\end{array}$ & $(0.099)$ \\
\hline 5000 & $\begin{array}{l}0.499 \\
(0.069)\end{array}$ & $\begin{array}{l}0.497 \\
(0.098)\end{array}$ & $\begin{array}{l}0.505 \\
(0.126)\end{array}$ & $\begin{array}{l}0.499 \\
(0.083)\end{array}$ & $\begin{array}{l}0.499 \\
(0.079)\end{array}$ & $\begin{array}{l}0.514 \\
(0.083)\end{array}$ & $(0.066)$ \\
\hline
\end{tabular}

can be calculated using the "cce" function in the "yuima" R package (http://r-forge.r-project.org/ projects/yuima). We used the default values of the "cce" function or values used in corresponding papers for parameters of estimators $\left(\theta=0.15\right.$ for PHY, $\theta=1$ for $\mathrm{MRC}_{1}, J=30, h^{-1}=10$ for LMM). Here we use the oracle estimator defined in [5] for LMM to avoid a complicated calculation. For the modulated realised covariance, we also examine an estimator $\mathrm{MRC}_{2}$ with $\theta=1 / 3$ which is used in Jacod et al. [19]. Table 2 shows the results of 1000 estimations. We used the same parameter values as above. Then the true value of the quadratic covariation becomes $\left\langle Y^{1}, Y^{2}\right\rangle_{T}=0.5$. For both cases of observation noise variance, we can see that sample standard deviations of our estimator are the best in large samples. The theoretical (asymptotic) minimum $\operatorname{Tn}^{-1 / 4}\left(\sigma_{3, *}^{2}\left(\Gamma_{1}^{-1}\right)_{11}+2 \sigma_{1, *} \sigma_{3, *}\left(\Gamma_{1}^{-1}\right)_{13}+\sigma_{1, *}^{2}\left(\Gamma_{1}^{-1}\right)_{33}\right)^{1 / 2}$ of standard deviations for all estimators is calculated in the last column of Table 2 . We can see that the sample standard deviations of MLE are close to the minima in large samples.

The asymptotic variance in (3.1) is always the best in this parametric setting. On the other hand, Bibinger et al. [5] gave a Cramér-Rao lower bound for one-dimensional perturbation and the asymptotic variance of LMM becomes optimal in that sense. The derivation of their bound is quite different from that of (3.1), and therefore, it is difficult to judge when these variances are the same even in this simple Brownian model. However, there is an interesting numerical result on this point. Let $V_{\text {MLE }}$ be the asymptotic variance of MLE for $\left\langle Y^{1}, Y^{2}\right\rangle_{T}$ and let $V_{\mathrm{LMM}}$ be that of LMM, and we set parameters as $T=1$, $\left(\lambda_{1}, \lambda_{2}\right)=(1,1),\left(\sigma_{1, *}, \sigma_{2, *}, \sigma_{3, *}\right)=\left(1, \sqrt{1-0.5^{2}}, 0.5\right)$ and $v_{*}=(0.001,0.001)$. Then we obtain $V_{\mathrm{MLE}}=T^{2}\left(\sigma_{3, *}^{2}\left(\Gamma_{1}^{-1}\right)_{11}+2 \sigma_{1, *} \sigma_{3, *}\left(\Gamma_{1}^{-1}\right)_{13}+\sigma_{1, *}^{2}\left(\Gamma_{1}^{-1}\right)_{33}\right)=0.1385502 \cdots$ and $V_{\mathrm{LMM}}=$ $0.1385502 \cdots$, that is, we see an exact numerical match between these variances. We have this agreement even if we set other values of parameters, and hence we expect that $V_{\mathrm{MLE}}=V_{\mathrm{LMM}}$ always holds for this model. 
However, the performances of MLE and LMM are different in above simulation because it is not easy to set parameters of LMM suitably. If we set $J>30$ for LMM, then the sample standard deviation is reduced, while the bias increase; for example, the average value of LMM is 0.51678 and the sample standard deviation is 0.05017 with $J=100, n=5000$ and $v_{*}=(0.001,0.001)$ in Table 2.

On the other hand, the asymptotic variances of LMM and MLE are different each other in a model with a time-dependent diffusion coefficient. Let the diffusion coefficient $b$ be defined by

$$
b(t, \sigma)=f(t)\left(\begin{array}{cc}
\sigma_{1} & 0 \\
\sigma_{3} & \sigma_{2}
\end{array}\right)
$$

for some smooth, positive function $f$ on $[0,1]$ with $T=1$, and let $\left\{S_{i}^{n, j}\right\}_{n, j, i}$ and $\left\{\varepsilon_{i}^{n, j}\right\}_{n, j, i}$ be the same as the above example. Then we can calculate asymptotic variances $\tilde{V}_{\text {MLE }}$ and $\tilde{V}_{\text {LMM }}$ of MLE and LMM, respectively, as

$$
\tilde{V}_{\mathrm{MLE}}=V_{\mathrm{MLE}} \times\left(\int_{0}^{1} f(t)^{2} d t\right)^{2} / \int_{0}^{1} f(t) d t, \quad \tilde{V}_{\mathrm{LMM}}=V_{\mathrm{LMM}} \times \int_{0}^{1} f(t)^{3} d t .
$$

The Cauchy-Schwarz inequality yields $\int_{0}^{1} f(t)^{3} d t \geq\left(\int_{0}^{1} f(t)^{2} d t\right)^{2} / \int_{0}^{1} f(t) d t$, and the equality is attained if and only if $f$ is a constant function. These results imply $\tilde{V}_{\text {MLE }}<\tilde{V}_{\text {LMM }}$ for any non-constant function $f$ if $V_{\mathrm{MLE}} \leq V_{\mathrm{LMM}}$. For example, let us set $f(t)=2-\sin (\pi t)$ to capture the U-shape of intra-day activities of a stock market. Then we obtain

$$
\tilde{V}_{\mathrm{MLE}} / \tilde{V}_{\mathrm{LMM}}=\frac{\left(\int_{0}^{1} f(t)^{2} d t\right)^{2}}{\int_{0}^{1} f(t) d t \int_{0}^{1} f(t)^{3} d t}=0.9533 \cdots
$$

if $V_{\mathrm{MLE}}=V_{\mathrm{LMM}}$. Thus, we can see the improvement of efficiency in a model with a timedependent $b$.

In the next, we consider the model with random diffusion coefficients and non-Gaussian noise. As mentioned in Remark 2.1, we cannot directly apply our results to stochastic volatility models. Here we consider the Cox-Ingersoll-Ross (CIR) process derived in [9] as a latent process with random diffusion coefficients. Let the latent process $Y$ satisfy

$$
d Y_{t}=\left(\begin{array}{c}
\alpha_{1}-\beta_{1} Y_{t}^{1} \\
\alpha_{2}-\beta_{2} Y_{t}^{2}
\end{array}\right) d t+\left(\begin{array}{cc}
\sigma_{1, *} \sqrt{Y_{t}^{1}} & 0 \\
\sigma_{3, *} \sqrt{Y_{t}^{2}} & \sigma_{2, *} \sqrt{Y_{t}^{2}}
\end{array}\right) d W_{t}
$$

where $\sigma_{*}=\left(\sigma_{1, *}, \sigma_{2, *}, \sigma_{3, *}\right) \in\left(\varepsilon^{\prime}, R^{\prime}\right) \times\left(-R^{\prime}, R^{\prime}\right) \times\left(\varepsilon^{\prime}, R^{\prime}\right)$. We assume Conditions $2 \alpha_{1}>$ $\sigma_{1, *}^{2}$ and $2 \alpha_{2}>\sigma_{2, *}^{2}+\sigma_{3, *}^{2}$ which ensure $Y_{t}^{1}>0$ and $Y_{t}^{2}>0$ for $t \in[0, T]$ almost surely. Let $\left\{\varepsilon_{i}^{n, j}\right\}_{i \in \mathbb{Z}}$ be i.i.d. random variables following a centered Gamma distribution with a shape parameter $k_{j}$ and a scale parameter $\theta_{j}$ for $j=1,2$. We define $\left\{N_{t}^{j}\right\}, \hat{v}_{n}, \hat{v}_{n}^{\prime}, \hat{\sigma}_{n}$, and $\hat{\sigma}_{n}^{\prime}$ similarly to the first example. We set the values of parameters as $k_{n}=\left[n^{5 / 8}\right], T=$ $1,\left(\lambda_{1}, \lambda_{2}\right)=(1,1),\left(\sigma_{1, *}, \sigma_{2, *}, \sigma_{3, *}\right)=\left(1, \sqrt{1-0.5^{2}}, 0.5\right),\left(\alpha_{1}, \alpha_{2}, \beta_{1}, \beta_{2}\right)=(1,1,1,1)$, and 
Table 3. Estimation errors of estimators of $\left\langle Y^{1}, Y^{2}\right\rangle_{T}$ for the CIR process

\begin{tabular}{lllllll}
\hline$n$ & MLE & PHY & MRC $_{1}$ & MRC $_{2}$ & QMLE & LMM \\
\hline 1000 & -0.0267 & -0.0063 & -0.0058 & -0.0036 & -0.0008 & -0.0348 \\
& $(0.0733)$ & $(0.1286)$ & $(0.1867)$ & $(0.1162)$ & $(0.1013)$ & $(0.0844)$ \\
5000 & -0.0023 & -0.0036 & -0.0022 & -0.0016 & -0.0005 & -0.0033 \\
& $(0.0456)$ & $(0.0858)$ & $(0.1305)$ & $(0.0768)$ & $(0.0580)$ & $(0.0719)$ \\
\hline
\end{tabular}

$\left(k_{1}, k_{2}, \theta_{1}, \theta_{2}\right)=(2,2, \sqrt{0.0005}, \sqrt{0.0005})$ which implies $v_{*}=(0.001,0.001)$. Table 3 shows averages and sample standard deviations of $T_{n}-\left\langle Y^{1}, Y^{2}\right\rangle_{T}$ for each estimator $T_{n}$ of the quadratic covariation $\left\langle Y^{1}, Y^{2}\right\rangle_{T}$ in 1000 simulations. $\left\langle Y^{1}, Y^{2}\right\rangle_{T}$ is random in this model since the diffusion coefficients are random. So we use extra-high-frequency observations $\left\{Y_{k / 100000}^{l}\right\}_{k=0}^{100000}$ of $Y$ to calculate the approximated true value of $\left\langle Y^{1}, Y^{2}\right\rangle_{T}$. In this model, we have not obtained the LAMN property nor asymptotic efficiency of our estimator though we expect to obtain them. However, we still see that our estimator achieves the best error variance in large samples.

\section{Asymptotically equivalent representation of the quasi-likelihood function}

We will prove our main results in the rest of this paper. In this section, we introduce an asymptotically equivalent representation $\tilde{H}_{n}(\sigma, v)$ of the quasi-likelihood function $H_{n}(\sigma, v)$, and prove the equivalence. $\tilde{H}_{n}$ is a useful function for deducing the limit of $H_{n}$.

\subsection{Some notations}

We denote $E_{m}$ as the $\mathcal{G}_{s_{m-1}}$-conditional expectation and $\bar{E}_{m}[\mathbf{X}]=\mathbf{X}-E_{m}[\mathbf{X}]$ for a random variable $\mathbf{X}$. We use the symbol $C$ for a generic positive constant that can vary from line to line.

For a sequence $c_{n}$ of positive-valued $\mathfrak{B}\left(\Pi_{n}\right)$-measurable random variables, let us denote by $\left\{\bar{R}_{n}\left(c_{n}\right)\right\}_{n \in \mathbb{N}},\left\{\underline{\mathrm{R}}_{n}\left(c_{n}\right)\right\}_{n \in \mathbb{N}}$ and $\left\{\dot{R}_{n}\left(c_{n}\right)\right\}_{n \in \mathbb{N}}$ sequences of random variables (which may depend on $1 \leq m \leq \ell_{n}$ and $\sigma$ ) satisfying

$$
\begin{aligned}
& E\left[\left(c_{n}^{-1}\left(r_{n} / b_{n}\right)^{-p_{1}}\left(b_{n} / \underline{\mathrm{r}}_{n}\right)^{-p_{2}}\left(\bar{k}_{n} / k_{n}\right)^{-p_{3}}\left(k_{n} / \underline{\mathrm{k}}_{n}\right)^{-p_{4}} b_{n}^{-\delta} \sup _{\sigma, m} E_{\Pi}\left[\left|\bar{R}_{n}\left(c_{n}\right)\right|^{q}\right]^{1 / q}\right)^{q^{\prime}}\right] \rightarrow 0, \\
& E\left[\left(c_{n}^{-1}\left(r_{n} / b_{n}\right)^{q_{1}}\left(b_{n} / \underline{\mathrm{r}}_{n}\right)^{q_{2}}\left(\bar{k}_{n} / k_{n}\right)^{q_{3}}\left(k_{n} / \underline{\mathrm{k}}_{n}\right)^{q_{4}} b_{n}^{\delta^{\prime}} \sup _{\sigma, m} E_{\Pi}\left[\left|\underline{\mathrm{R}}_{n}\left(c_{n}\right)\right|^{q}\right]^{1 / q}\right)^{q^{\prime}}\right] \rightarrow 0
\end{aligned}
$$

and

$$
c_{n}^{-1}\left(r_{n} / b_{n}\right)^{q_{1}}\left(b_{n} / \underline{\mathrm{r}}_{n}\right)^{q_{2}}\left(\bar{k}_{n} / k_{n}\right)^{q_{3}}\left(k_{n} / \underline{\mathrm{k}}_{n}\right)^{q_{4}} \sup _{\sigma, m}\left|\dot{R}_{n}\left(c_{n}\right)\right| \rightarrow^{p} 0,
$$

respectively, as $n \rightarrow \infty$ for any $\delta, q, q^{\prime}, q_{1}, \ldots, q_{4}>0$ with some constants $\delta^{\prime}, p_{1}, \ldots, p_{4} \geq 0$. 
Let $M_{m}(v)=\operatorname{diag}\left(v_{1} M_{1, m}, v_{2} M_{2, m}\right)$ for $v=\left(v_{1}, v_{2}\right), \tilde{b}_{m}^{k}=b^{k}\left(s_{m-1}, X_{s_{m-1}}, \sigma\right), \tilde{b}_{m, *}^{k}=$ $b^{k}\left(s_{m-1}, X_{s_{m-1}}, \sigma_{*}\right)$,

$$
\begin{aligned}
& \tilde{Z}_{m}=\left(\left(\left(\tilde{b}_{m, *}^{1} \cdot\left(W_{S_{i}^{n, 1}}-W_{S_{i-1}^{n, 1}}\right)+\varepsilon_{i}^{n, 1}-\varepsilon_{i-1}^{n, 1}\right)_{i=K_{m-1}^{1}+2}^{K_{m}^{1}}\right)^{\top},\right. \\
&\left.\left(\left(\tilde{b}_{m, *}^{2} \cdot\left(W_{S_{j}^{n, 2}}-W_{S_{j-1}^{n, 2}}\right)+\varepsilon_{j}^{n, 2}-\varepsilon_{j-1}^{n, 2}\right)_{j=K_{m-1}^{2}+2}^{K_{m}^{2}}\right)^{\top}\right)^{\top}, \\
& \tilde{S}_{m}(\sigma, v)=\left(\begin{array}{cc}
\operatorname{diag}\left(\left(\left|\tilde{b}_{m}^{1}\right|^{2}\left|I_{i, m}^{1}\right|\right)_{i}\right) & \left\{\tilde{b}_{m}^{1} \cdot \tilde{b}_{m}^{2}\left|I_{i, m}^{1} \cap I_{j, m}^{2}\right|\right\}_{i j} \\
\left\{\tilde{b}_{m}^{1} \cdot \tilde{b}_{m}^{2}\left|I_{i, m}^{1} \cap I_{j, m}^{2}\right|\right\}_{j i} & \operatorname{diag}\left(\left(\left|\tilde{b}_{m}^{2}\right|^{2}\left|I_{j, m}^{2}\right|\right)_{j}\right)
\end{array}\right)+M_{m}(v),
\end{aligned}
$$

and

$$
\tilde{H}_{n}(\sigma, v)=-\frac{1}{2} \sum_{m=2}^{\ell_{n}} \tilde{Z}_{m}^{\top} \tilde{S}_{m}^{-1}(\sigma, v) \tilde{Z}_{m}-\frac{1}{2} \sum_{m=2}^{\ell_{n}} \log \operatorname{det} \tilde{S}_{m}(\sigma, v) .
$$

The diffusion coefficient $b$ in $\tilde{Z}_{m}$ and $\tilde{S}_{m}$ are either $b\left(s_{m-1}, X_{s_{m-1}}, \sigma\right)$ or $b\left(s_{m-1}, X_{s_{m-1}}, \sigma_{*}\right)$. Hence, we do not need to consider the time-dependent structure of $b$ when we study asymptotics of the summands in $\tilde{H}_{n}$. In particular, we obtain $E_{m}\left[\tilde{Z}_{m}^{\top} \partial_{\sigma} \tilde{S}_{m}\left(\sigma_{*}, v_{*}\right)^{-1} \tilde{Z}_{m}+\right.$ $\left.\partial_{\sigma} \log \operatorname{det} \tilde{S}_{m}\left(\sigma_{*}, v_{*}\right)\right]=0$ by $\partial_{\sigma} \log \operatorname{det} \tilde{S}_{m}(\sigma, v)=\operatorname{tr}\left(\partial_{\sigma} \tilde{S}_{m} \tilde{S}_{m}^{-1}\right)(\sigma, v)$. We will prove the asymptotic equivalence of $H_{n}$ and $\tilde{H}_{n}$ and then investigate asymptotic properties of $\tilde{H}_{n}$ instead of $H_{n}$.

Similarly to the approach of Gloter and Jacod [13], we can use the following stronger condition $\left(\mathrm{A} 1^{\prime}\right)$ instead of (A1) in several part of the proof. Then localization techniques and Girsanov's theorem enable us to replace $\left(\mathrm{A} 1^{\prime}\right)$ with $(\mathrm{A} 1)$.

Assumption (A1'). Assumption (A1) is satisfied, $O=\mathbb{R}^{d_{2}}, \sup _{t, x, \sigma}\left\|\left(b b^{\top}\right)^{-1}\right\|(t, x, \sigma)<\infty$, $\mu_{t} \equiv 0$ and $Y_{0}, \sup _{t}\left(\left|b_{t}^{(l)}\right| \vee\left|\hat{b}_{t}^{(l)}\right|\right)$, and $\partial_{t}^{i} \partial_{x}^{j} \partial_{\sigma}^{k} b$ are all bounded for $l=0,1,0 \leq 2 i+j \leq 4$ and $0 \leq k \leq 4$.

We can also see that $\left(\mathrm{A} 1^{\prime}\right)$ implies (B1).

\subsection{Fundamental properties of the noise covariance matrix}

In the following subsection, we will show the asymptotic equivalence of $\tilde{H}_{n}$ and $H_{n}$, namely that

$$
b_{n}^{-1 / 2} \sup _{\sigma \in \Lambda}\left|\partial_{\sigma}^{j}\left(H_{n}\left(\sigma, \hat{v}_{n}\right)-H_{n}\left(\sigma_{*}, \hat{v}_{n}\right)\right)-\partial_{\sigma}^{j}\left(\tilde{H}_{n}\left(\sigma, v_{*}\right)-\tilde{H}_{n}\left(\sigma_{*}, v_{*}\right)\right)\right| \stackrel{p}{\rightarrow} 0
$$

as $n \rightarrow \infty$ for $0 \leq j \leq 3$. To that end, we first show fundamental properties of $S_{m}$ and $\tilde{S}_{m}$. These matrices inherit some properties of $M_{j, m}$, that are necessary to deduce the limit of $H_{n}$ and $\tilde{H}_{n}$. The first property (4.2) concerns the trace of a matrix related to $M_{j, m}$ investigated by [13]. In the one-dimensional model with noisy, equidistance observations, this property can be directly applied to the quasi-likelihood function because the covariance matrix of the latent 
process is the unit matrix. However, this is insufficient for our purpose because our covariance matrix $S_{m}(\sigma, v)-M_{m}(v)$ of the latent process is rather complicated. Therefore, we investigate further matrix properties related to $M_{j, m}$.

First, we consider the results in [13]. For any positive constants $p, q, a$ and $b$, eigenvalues of $\left(a \mathcal{E}+M_{j, m}\right)^{-1}$ are $\left\{\left(a+2\left(1-\cos \left(i \pi\left(k_{m}^{j}+1\right)^{-1}\right)\right)\right\}_{i=1}^{k_{m}^{j}}\right.$ and we obtain

$$
\begin{aligned}
\pi^{-1}\left(k_{m}^{j}+1\right) I_{p}(a)-a^{-p} & \leq \operatorname{tr}\left(\left(a \mathcal{E}+M_{j, m}\right)^{-p}\right) \leq \pi^{-1}\left(k_{m}^{j}+1\right) I_{p}(a), \\
\pi^{-1}\left(k_{m}^{j}+1\right) I_{p, q}(a, b)-a^{-p} b^{-q} & \leq \operatorname{tr}\left(\left(a \mathcal{E}+M_{j, m}\right)^{-p}\left(b \mathcal{E}+M_{j, m}\right)^{-q}\right) \\
& \leq \pi^{-1}\left(k_{m}^{j}+1\right) I_{p, q}(a, b),
\end{aligned}
$$

where $I_{p}(a)=\int_{0}^{\pi}(a+2(1-\cos x))^{-p} d x$ and $I_{p, q}(a, b)=\int_{0}^{\pi}(a+2(1-\cos x))^{-p}(b+2(1-$ $\cos x))^{-q} d x$. Simple calculations show that $I_{1}(a)=\pi / \sqrt{a(4+a)}, I_{2}(a)=\pi(2+a) a^{-3 / 2}(4+$ $a)^{-3 / 2}$ and $\int_{0}^{\pi}\{\log (a+2(1-\cos x))-\log (b+2(1-\cos x))\} d x=2 \pi(\log (\sqrt{a}+\sqrt{4+a})-$ $\log (\sqrt{b}+\sqrt{4+b}))$. See Section 4.1 in [13] for the details. Moreover, differentiation with respect to $a$ yields

$$
I_{p}(a)=\frac{(-1)^{p-1}}{(p-1) !}\left(\frac{d}{d a}\right)^{p-1}\left(\frac{\pi}{\sqrt{a(4+a)}}\right) .
$$

In particular, if $a=\mathbf{X}_{n} b_{n}^{-1}$ for some tight random variables $\left\{\mathbf{X}_{n}\right\}_{n}$, then we have

$$
I_{p}\left(\mathbf{X}_{n} b_{n}^{-1}\right)=\frac{\pi(2 p-3) ! !}{2^{p}(p-1) !}\left(\mathbf{X}_{n} b_{n}^{-1}\right)^{-p+1 / 2}+O_{p}\left(b_{n}^{p-3 / 2}\right) .
$$

For $\varepsilon \geq 0$, let $\left\{p_{j}(\varepsilon)\right\}_{j \in \mathbb{N}}$ and $\left\{p_{j}^{\prime}(\varepsilon)\right\}_{j \in \mathbb{N}}$ be sequences of positive numbers satisfying $p_{1}(\varepsilon)=$ $2+\varepsilon, p_{1}^{\prime}(\varepsilon)=1+\varepsilon, p_{j+1}(\varepsilon)=2+\varepsilon-1 / p_{j}(\varepsilon)$, and $p_{j+1}^{\prime}(\varepsilon)=2+\varepsilon-1 / p_{j}^{\prime}(\varepsilon)$ for $j \in \mathbb{N}$. Let $E_{i, j}(a)$ be a $k_{m}^{j} \times k_{m}^{j}$ matrix satisfying $\left(E_{i, j}(a)\right)_{k, l}=\delta_{k l}+a \delta_{i k} \delta_{j l}$ for $a \in \mathbb{R}$. Then we have that $E_{k_{m}^{j}, k_{m}^{j}-1}\left(p_{k_{m}^{j}-1}(\varepsilon)^{-1}\right) \cdots E_{2,1}\left(p_{1}(\varepsilon)^{-1}\right)\left(\varepsilon \mathcal{E}+M_{j, m}\right) E_{1,2}\left(p_{1}(\varepsilon)^{-1}\right) \cdots E_{k_{m}^{j}-1, k_{m}^{j}}\left(p_{k_{m}^{j}-1}(\varepsilon)^{-1}\right)$ is equal to $\operatorname{diag}\left(\left(p_{j}(\varepsilon)\right)_{j=1}^{k_{m}^{j}}\right)$, and hence

$$
\begin{aligned}
\left(\varepsilon \mathcal{E}+M_{j, m}\right)^{-1}= & \left\{\prod_{k+1 \leq i \leq l} p_{i-1}(\varepsilon)^{-1} 1_{\{k \leq l\}}\right\}_{k, l} \\
& \times \operatorname{diag}\left(\left(p_{j}(\varepsilon)^{-1}\right)_{j=1}^{k_{m}^{j}}\right)\left\{\prod_{l+1 \leq i \leq k} p_{i-1}(\varepsilon)^{-1} 1_{\{l \leq k\}}\right\}_{k, l} .
\end{aligned}
$$

Moreover, we have the following lemma.

Lemma 4.1. Let $\varepsilon \in[0,1)$ and $p_{+}(\varepsilon)=1+\varepsilon / 2+\sqrt{\varepsilon+\varepsilon^{2} / 4}$. Then 
1. $1 \leq p_{j}^{\prime}(\varepsilon) \leq p_{+}(\varepsilon)<p_{j}(\varepsilon) \leq 1+1 / j+j \varepsilon$ for $j \in \mathbb{N},\left\{p_{j}(\varepsilon)\right\}_{j}$ is monotone decreasing, and $\left\{p_{j}^{\prime}(\varepsilon)\right\}_{j}$ is monotone nondecreasing.

2. $\left\{\left(\left(\varepsilon \mathcal{E}+M_{j, m}\right)^{-1}\right)_{k k}\right\}_{k=1}^{\left[k_{m}^{j} / 2\right]}$ is monotone increasing.

3. $p_{j}-p_{+} \leq(1+\sqrt{\varepsilon})^{-(j-2)}$ and $p_{+}-p_{j}^{\prime} \leq \sqrt{\varepsilon}(1+\sqrt{\varepsilon})^{-(j-1)}$ for $j \geq 2$.

4. $\prod_{j=1}^{k} p_{j}^{\prime}(\varepsilon)=\left(p_{k}(\varepsilon)-1\right) \prod_{j=1}^{k-1} p_{j}(\varepsilon)$ for any $k \geq 2$.

Proof. 1. We simply denote $p_{j}=p_{j}(\varepsilon)$ and $p_{+}=p_{+}(\varepsilon)$. We will prove $p_{+}<p_{j} \leq 1+1 / j+j \varepsilon$ for $j \in \mathbb{N}$ by induction. The results obviously hold for $j=1$. Assume the results hold for all values in $\mathbb{N}$ up to $j$. Then since $p_{+}=2+\varepsilon-1 / p_{+}$, we obtain $p_{j+1}-p_{+}=1 / p_{+}-1 / p_{j}>0$, and

$$
\begin{aligned}
p_{j+1} & \leq 2+\varepsilon-(j /(j+1))\left(1+j^{2} \varepsilon /(j+1)\right)^{-1} \leq 2+\varepsilon-(j /(j+1))\left(1-j^{2} \varepsilon /(j+1)\right) \\
& \leq 1+1 /(j+1)+(j+1) \varepsilon .
\end{aligned}
$$

Hence, we have $p_{+}<p_{j} \leq 1+1 / j+j \varepsilon$ for $j \in \mathbb{N}$. Moreover, we can inductively deduce $p_{j+1}-$ $p_{j}=1 / p_{j-1}-1 / p_{j}<0$. The results for $\left\{p_{j}^{\prime}(\varepsilon)\right\}_{j}$ are obtained similarly.

2. By considering the cofactor matrix and (4.4), we have

$$
\begin{aligned}
\left(\left(\varepsilon \mathcal{E}+M_{j, m}\right)^{-1}\right)_{k k} & =\frac{\operatorname{det}\left(\varepsilon \mathcal{E}_{k-1}+M(k-1)\right) \operatorname{det}\left(\varepsilon \mathcal{E}_{k_{m}^{j}-k}+M\left(k_{m}^{j}-k\right)\right)}{\operatorname{det}\left(\varepsilon \mathcal{E}+M_{j, m}\right)} \\
& =\frac{\prod_{l=1}^{k-1} p_{l} \prod_{l=1}^{k_{m}^{j}-k} p_{l}}{\prod_{l=1}^{k_{m}^{j}} p_{l}}
\end{aligned}
$$

Therefore, we obtain the result by monotonicity of $p_{j}$.

3. This is easy since $p_{j}-p_{+}=\left(p_{j-1}-p_{+}\right) / p_{+} p_{j-1} \leq\left(p_{1}-p_{+}\right) / p_{+}^{j-1} \leq p_{+}^{-j+2}$. 4.

$$
\begin{aligned}
\prod_{j=1}^{k} p_{j}^{\prime}(\varepsilon) & =\operatorname{det}\left(\varepsilon \mathcal{E}+M(k)-\left(E_{11}(1)-\mathcal{E}\right)\right)=\operatorname{det}\left(\varepsilon \mathcal{E}+M(k)-\left(E_{k k}(1)-\mathcal{E}\right)\right) \\
& =\left(p_{k}(\varepsilon)-1\right) \prod_{j=1}^{k-1} p_{j}(\varepsilon)
\end{aligned}
$$

Let $\bar{\rho}=\sup _{t, \sigma}\left(\left|b^{1} \cdot b^{2}\right|\left|b^{1}\right|^{-1}\left|b^{2}\right|^{-1}\right)\left(t, X_{t}, \sigma\right), \tilde{D}_{m}=\left(\tilde{D}_{1, m}, \tilde{D}_{2, m}\right), \tilde{D}_{j, m}=\operatorname{diag}\left(\left(\left|\tilde{b}_{m}^{j}\right|^{2} \times\right.\right.$ $\left.\left.\left|I_{i, m}^{j}\right|\right)_{i}\right)+v_{j, *} M_{j, m}, D_{m}^{\prime}=\left(D_{1, m}^{\prime}, D_{2, m}^{\prime}\right), D_{j, m}^{\prime}=\operatorname{diag}\left(\left(\left|I_{i, m}^{j}\right|\right)_{i}\right)$, and $\check{D}_{j, m}=\left|\tilde{b}_{m}^{j}\right|^{2} r_{n} \mathcal{E}+$ $v_{j, *} M_{j, m}$.

Lemma 4.2. Assume (B1). Then $\operatorname{tr}\left(\tilde{S}_{m}^{-1}\left(\sigma, v_{*}\right)\right)=\bar{R}_{n}\left(b_{n}^{1 / 2} k_{n}\right)$. 
Proof. Let $D_{m}^{\prime \prime}=\operatorname{diag}\left(\left|\tilde{b}_{m}^{1}\right|^{2} D_{1, m}^{\prime},\left|\tilde{b}_{m}^{2}\right|^{2} D_{2, m}^{\prime}\right)$ and $D_{m}^{\prime \prime \prime}=\left(D_{m}^{\prime \prime}\right)^{-1 / 2} \tilde{D}_{m}\left(D_{m}^{\prime \prime}\right)^{-1 / 2}$, then we have

$$
\begin{aligned}
\tilde{S}_{m}= & \left(D_{m}^{\prime \prime}\right)^{1 / 2}\left(D_{m}^{\prime \prime \prime}\right)^{1 / 2}\left(\mathcal{E}+\left(D_{m}^{\prime \prime \prime}\right)^{-1 / 2}\left(D_{m}^{\prime \prime}\right)^{-1 / 2}\left(\tilde{S}_{m}-\tilde{D}_{m}\right)\left(D_{m}^{\prime \prime}\right)^{-1 / 2}\right. \\
& \left.\times\left(D_{m}^{\prime \prime \prime}\right)^{-1 / 2}\right)\left(D_{m}^{\prime \prime \prime}\right)^{1 / 2}\left(D_{m}^{\prime \prime}\right)^{1 / 2}
\end{aligned}
$$

Moreover, Lemma A.4, (B1), and Lemma 2 in [25] yield

$$
\begin{aligned}
& \left\|\left(D_{m}^{\prime \prime \prime}\right)^{-1 / 2}\left(D_{m}^{\prime \prime}\right)^{-1 / 2}\left(\tilde{S}_{m}-\tilde{D}_{m}\right)\left(D_{m}^{\prime \prime}\right)^{-1 / 2}\left(D_{m}^{\prime \prime \prime}\right)^{-1 / 2}\right\| \\
& \quad \leq\left\|\left(D_{m}^{\prime \prime}\right)^{-1 / 2}\left(\tilde{S}_{m}-\tilde{D}_{m}\right)\left(D_{m}^{\prime \prime}\right)^{-1 / 2}\right\| \\
& \quad \leq \bar{\rho}\left\{\left\|\left\{\frac{\left|I_{i, m}^{1} \cap I_{j, m}^{2}\right|}{\left|I_{i, m}^{1}\right|^{1 / 2}\left|I_{j, m}^{2}\right|^{1 / 2}}\right\}_{i, j}\right\| \vee\left\|\left\{\frac{\left|I_{i, m}^{1} \cap I_{j, m}^{2}\right|}{\left|I_{i, m}^{1}\right|^{1 / 2}\left|I_{j, m}^{2}\right|^{1 / 2}}\right\}_{j, i}\right\|\right\} \leq \bar{\rho}<1 .
\end{aligned}
$$

Therefore, we obtain

$$
\begin{aligned}
\operatorname{tr}\left(\tilde{S}_{m}^{-1}\right) \leq & \operatorname{tr}\left(\left(D_{m}^{\prime \prime \prime}\right)^{-1 / 2}\left(D_{m}^{\prime \prime}\right)^{-1}\left(D_{m}^{\prime \prime \prime}\right)^{-1 / 2}\right) \\
& \times\left\|\left(\mathcal{E}+\left(D_{m}^{\prime \prime \prime}\right)^{-1 / 2}\left(D_{m}^{\prime \prime}\right)^{-1 / 2}\left(\tilde{S}_{m}-\tilde{D}_{m}\right)\left(D_{m}^{\prime \prime}\right)^{-1 / 2}\left(D_{m}^{\prime \prime \prime}\right)^{-1 / 2}\right)^{-1}\right\| \\
\leq & \operatorname{tr}\left(\tilde{D}_{m}^{-1}\right) /(1-\bar{\rho}) \leq \sum_{j=1}^{2} \operatorname{tr}\left(\check{D}_{j, m}^{-1}\right) r_{n} \underline{r}_{n}^{-1}(1-\bar{\rho})^{-1},
\end{aligned}
$$

by Lemma A.1, the equation $\tilde{D}_{j, m}=\check{D}_{j, m}^{1 / 2}\left(\mathcal{E}-\check{D}_{j, m}^{-1 / 2}\left(\check{D}_{j, m}-\tilde{D}_{j, m}\right) \check{D}_{j, m}^{-1 / 2}\right) \check{D}_{j, m}^{1 / 2}$, and that

$$
\left\|\check{D}_{j, m}^{-1 / 2}\left(\check{D}_{j, m}-\tilde{D}_{j, m}\right) \check{D}_{j, m}^{-1 / 2}\right\| \leq\left(\left|\tilde{b}_{m}^{j}\right|^{2} r_{n}\right)^{-1}\left|\tilde{b}_{m}^{j}\right|^{2}\left(r_{n}-\underline{\mathrm{r}}_{n}\right)=1-\underline{\mathrm{r}}_{n} / r_{n}
$$

We thus obtain the desired results by (4.2).

\subsection{Asymptotic equivalence of $H_{n}$ and $\tilde{H}_{n}$}

In this section, we prove the asymptotic equivalence of $H_{n}$ and $\tilde{H}_{n}$. We provide the following lemma about estimates of moments of the quantities related to $H_{n}$ and $\tilde{H}_{n}$. The proof is given in the appendix; it is obtained based on the properties of $M_{j, m}$ in Section 4.2, standard Itô calculus, and some results from linear algebra.

Let $\tilde{S}_{m, *}=\tilde{S}_{m}\left(\sigma_{*}, v_{*}\right)$.

Lemma 4.3. Assume (B1). Let $\mathbf{S}=\mathbf{S}_{n, m}$ be a symmetric, $\mathcal{G}_{s_{m-1}}$-measurable random matrix of size $k_{m}^{1}+k_{m}^{2}$ satisfying $\left\|\tilde{S}_{m, *} \mathbf{S} \tilde{S}_{m, *}\right\| \leq b_{n}^{-1}$ for $n \in \mathbb{N}$ and $1 \leq m \leq \ell_{n}$. Then there exists a positive random sequence $\left\{Q_{n, q}\right\}_{q \geq 2}$ which does not depend on $\mathbf{S}$ such that $Q_{n, q}=\bar{R}_{n}(1)$ for $q \geq 2$ and

1. $\left|E_{m}\left[\left(\tilde{Z}_{m}^{\top} \mathbf{S} \tilde{Z}_{m}\right)^{2}\right]-2 \operatorname{tr}\left(\left(\mathbf{S} \tilde{S}_{m, *}\right)^{2}\right)-\operatorname{tr}\left(\mathbf{S} \tilde{S}_{m, *}\right)^{2}\right| \leq Q_{n, 2}, E_{m}\left[\left(\tilde{Z}_{m}^{\top} \mathbf{S} \tilde{Z}_{m}\right)^{4}\right] \leq\left(\left(b_{n}^{-4} k_{n}^{7}\right) \vee\right.$ $\left.\left(b_{n}^{-2} k_{n}^{4}\right)\right) Q_{n, 4}$ and $E_{m}\left[\left|\tilde{Z}_{m}^{\top} \mathbf{S} \tilde{Z}_{m}\right|^{q}\right] \leq b_{n}^{-q} k_{n}^{2 q} Q_{n, q}$ for $q>4$. 
2. $E_{\Pi}\left[\left|\sum_{m}\left(Z_{m}-\tilde{Z}_{m}\right)^{\top} \mathbf{S}\left(Z_{m}+\tilde{Z}_{m}\right)\right|^{q}\right] \leq\left(b_{n}^{-3} k_{n}^{7}\right)^{q / 4} Q_{n, q}$ for $q \geq 4$.

3. $E_{\Pi}\left[\left|\sum_{m}\left(Z_{m}-\tilde{Z}_{m}\right)^{\top} \mathbf{S}\left(Z_{m}+\tilde{Z}_{m}\right)\right|^{2}\right] \leq b_{n}^{-1} k_{n}^{2} Q_{n, 2}$.

Proof. See the appendix.

Now we obtain the asymptotic equivalence of $H_{n}$ and $\tilde{H}_{n}$.

Lemma 4.4. Assume (B1), (A2) and (V). Then

$$
b_{n}^{-1 / 2} \sup _{\sigma \in \Lambda}\left|\partial_{\sigma}^{j}\left(H_{n}\left(\sigma, \hat{v}_{n}\right)-H_{n}\left(\sigma_{*}, \hat{v}_{n}\right)\right)-\partial_{\sigma}^{j}\left(\tilde{H}_{n}\left(\sigma, v_{*}\right)-\tilde{H}_{n}\left(\sigma_{*}, v_{*}\right)\right)\right| \rightarrow^{p} 0
$$

and $b_{n}^{-1 / 4}\left(\partial_{\sigma} H_{n}\left(\sigma_{*}, \hat{v}_{n}\right)-\partial_{\sigma} \tilde{H}_{n}\left(\sigma_{*}, v_{*}\right)\right) \rightarrow^{p} 0$ as $n \rightarrow \infty$ for $0 \leq j \leq 3$. If (B4) holds as well, then

$$
\begin{aligned}
& E_{\Pi}\left[\left(b_{n}^{-1 / 2} \sup _{\sigma \in \Lambda}\left|\partial_{\sigma}^{j}\left(H_{n}\left(\sigma, \hat{v}_{n}\right)-H_{n}\left(\sigma_{*}, \hat{v}_{n}\right)\right)-\partial_{\sigma}^{j}\left(\tilde{H}_{n}\left(\sigma, v_{*}\right)-\tilde{H}_{n}\left(\sigma_{*}, v_{*}\right)\right)\right|\right)^{q}\right] \\
& \quad=\bar{R}_{n}\left(\left(b_{n}^{-5} k_{n}^{7}\right)^{q / 4}\right)
\end{aligned}
$$

for any $0 \leq j \leq 3$ and $q>0$.

Proof. We first obtain

$$
\begin{aligned}
& H_{n}\left(\sigma, v_{*}\right)-H_{n}\left(\sigma_{*}, v_{*}\right)-\left(\tilde{H}_{n}\left(\sigma, v_{*}\right)-\tilde{H}_{n}\left(\sigma_{*}, v_{*}\right)\right) \\
&=-\frac{1}{2} \sum_{m}\left\{\left(Z_{m}-\tilde{Z}_{m}\right)^{\top}\left(S_{m}^{-1}\left(\sigma, v_{*}\right)-S_{m}^{-1}\left(\sigma_{*}, v_{*}\right)\right)\left(Z_{m}+\tilde{Z}_{m}\right)\right. \\
& \quad+\tilde{Z}_{m}^{\top}\left(S_{m}^{-1}\left(\sigma, v_{*}\right)-S_{m}^{-1}\left(\sigma_{*}, v_{*}\right)-\tilde{S}_{m}^{-1}\left(\sigma, v_{*}\right)+\tilde{S}_{m}^{-1}\left(\sigma_{*}, v_{*}\right)\right) \tilde{Z}_{m} \\
&\left.\quad+\left(\log \frac{\operatorname{det} S_{m}\left(\sigma, v_{*}\right)}{\operatorname{det} \tilde{S}_{m}\left(\sigma, v_{*}\right)}-\log \frac{\operatorname{det} S_{m}\left(\sigma_{*}, v_{*}\right)}{\operatorname{det} \tilde{S}_{m}\left(\sigma_{*}, v_{*}\right)}\right)\right\} \\
&=: \hat{\Psi}_{1, n}(\sigma)+\hat{\Psi}_{2, n}(\sigma)+\hat{\Psi}_{3, n}(\sigma) .
\end{aligned}
$$

We will give estimates for these quantities. Point 2 of Lemma 4.3 yields $\sup _{\sigma} E_{\Pi}\left[\mid b_{n}^{-1 / 2} \times\right.$ $\left.\left.\partial_{\sigma}^{j} \hat{\Psi}_{1, n}\right|^{q}\right]=\bar{R}_{n}\left(\left(b_{n}^{-5} k_{n}^{7}\right)^{q / 4}\right)$ for $0 \leq j \leq 4$ and $q>0$, and consequently by Sobolev's inequality $E_{\Pi}\left[\sup _{\sigma}\left|b_{n}^{-1 / 2} \partial_{\sigma}^{j} \hat{\Psi}_{1, n}\right|^{q}\right]=\bar{R}_{n}\left(\left(b_{n}^{-5} k_{n}^{7}\right)^{q / 4}\right)$ as $n \rightarrow \infty$ for $0 \leq j \leq 3$ and $q>d$.

Let

$\mathbf{C}(x, \sigma, v)$

$$
=\left(\begin{array}{cc}
\left\{\left|b^{1}\left(s_{m-1}, x, \sigma\right)\right|^{2}\left|I_{i, m}^{1}\right| \delta_{i i^{\prime}}\right\}_{i i^{\prime}}+v_{1} M_{1, m} & \left\{b^{1} \cdot b^{2}\left(s_{m-1}, x, \sigma\right)\left|I_{i, m}^{1} \cap I_{j, m}^{2}\right|\right\}_{i j} \\
\left\{b^{1} \cdot b^{2}\left(s_{m-1}, x, \sigma\right)\left|I_{i, m}^{1} \cap I_{j, m}^{2}\right|\right\}_{j i} & \left\{\left|b^{2}\left(s_{m-1}, x, \sigma\right)\right|^{2}\left|I_{j, m}^{2}\right| \delta_{j j^{\prime}}\right\}_{j j^{\prime}}+v_{2} M_{2, m}
\end{array}\right)
$$


for $v=\left(v_{1}, v_{2}\right), \tilde{\mathbf{C}}(x, v)=\mathbf{C}(x, \sigma, v)^{-1}-\mathbf{C}\left(x, \sigma_{*}, v\right)^{-1}, \mathbf{C}^{(1)}=\int_{0}^{1} \partial_{x} \tilde{\mathbf{C}}\left(t \hat{X}_{m-1}+(1-\right.$ t) $\left.X_{s_{m-1}}, v_{*}\right) d t, \mathbf{C}^{(2)}=\partial_{x} \tilde{\mathbf{C}}\left(X_{s_{m-2}}, v_{*}\right)$ and $\mathbf{C}^{(3)}=\mathbf{C}\left(X_{s_{m-2}}, \sigma_{*}, v_{*}\right)$. Then we obtain

$$
\begin{aligned}
E_{\Pi}\left[\left|\hat{\Psi}_{2, n}\right|^{q}\right]= & 2^{-q} E_{\Pi}\left[\left|\sum_{m} \tilde{Z}_{m}^{\top} \mathbf{C}^{(1)} \tilde{Z}_{m}\left(\hat{X}_{m-1}-X_{s_{m-1}}\right)\right|^{q}\right] \\
\leq & C E_{\Pi}\left[\left|\sum_{m} \operatorname{tr}\left(\mathbf{C}^{(1)} E_{m}\left[\tilde{Z}_{m} \tilde{Z}_{m}^{\top}\right]\right)\left(\hat{X}_{m-1}-X_{s_{m-1}}\right)\right|^{q}\right] \\
& +C E_{\Pi}\left[\left|\sum_{m} \operatorname{tr}\left(\mathbf{C}^{(1)} \bar{E}_{m}\left[\tilde{Z}_{m} \tilde{Z}_{m}^{\top}\right]\right)^{2}\left(\hat{X}_{m-1}-X_{s_{m-1}}\right)^{2}\right|^{q / 2}\right] \\
\leq & C E_{\Pi}\left[\left|\sum_{m} \operatorname{tr}\left(\mathbf{C}^{(2)} \mathbf{C}^{(3)}\right)\left(\hat{X}_{m-1}-X_{s_{m-1}}\right)\right|^{q}\right]+O_{p}\left(\left(\ell_{n} b_{n}^{-1 / 2} k_{n} \ell_{n}^{-1}\right)^{q} b_{n}^{\varepsilon}\right) \\
& +O_{p}\left(\ell_{n}^{q / 2} b_{n}^{-q} k_{n}^{2 q} \ell_{n}^{-q / 2} b_{n}^{\varepsilon}\right) \\
= & O_{p}\left(b_{n}^{\varepsilon} \ell_{n}^{q / 2} b_{n}^{-q / 2} k_{n}^{q} \ell_{n}^{-q / 2}\right)+O_{p}\left(b_{n}^{\varepsilon} b_{n}^{-q / 2} k_{n}^{q}\right)+O_{p}\left(b_{n}^{\varepsilon} b_{n}^{-q} k_{n}^{2 q}\right) \\
= & O_{p}\left(b_{n}^{-q+\varepsilon} k_{n}^{2 q}\right)
\end{aligned}
$$

for any $q>0$ and $\varepsilon>0$, by Lemma 4.3, the Burkholder-Davis-Gundy inequality, $\sup _{m} E_{\Pi}\left[\left|E_{m-1}\left[\hat{X}_{m-1}-X_{s_{m-1}}\right]\right|^{p}\right]^{1 / p}=O_{p}\left(\ell_{n}^{-1} b_{n}^{\varepsilon}\right)$ and $\sup _{m} E_{\Pi}\left[\left|\hat{X}_{m-1}-X_{s_{m-1}}\right|^{q}\right]^{1 / q}=$ $O_{p}\left(\ell_{n}^{-1 / 2} b_{n}^{\varepsilon}\right)$. Similar estimates for $\partial_{\sigma}^{j} \hat{\Psi}_{2, n}$ and Sobolev's inequality yield $E_{\Pi}\left[\sup _{\sigma} \mid b_{n}^{-1 / 2} \times\right.$ $\left.\left.\partial_{\sigma}^{j} \hat{\Psi}_{2, n}\right|^{q}\right]=O_{p}\left(b_{n}^{-3 q / 2+\varepsilon} k_{n}^{2 q}\right)$ for $0 \leq j \leq 3, q>0$ and any $\varepsilon>0$. If (B4) is satisfied, we obtain $E_{\Pi}\left[\sup _{\sigma}\left|b_{n}^{-1 / 2} \partial_{\sigma}^{j} \hat{\Psi}_{2, n}\right|^{q}\right]=\bar{R}_{n}\left(b_{n}^{-3 q / 2} k_{n}^{2 q}\right)$.

Similarly, we have $E_{\Pi}\left[\sup _{\sigma}\left|b_{n}^{-1 / 2} \partial_{\sigma}^{j} \hat{\Psi}_{3, n}\right|^{q}\right]=O_{p}\left(b_{n}^{-q+\varepsilon} k_{n}^{q}\right)$, and therefore we obtain $b_{n}^{-1 / 2} \sup _{\sigma}\left|\partial_{\sigma}^{j}\left(H_{n}\left(\sigma, v_{*}\right)-H_{n}\left(\sigma_{*}, v_{*}\right)\right)-\partial_{\sigma}^{j}\left(\tilde{H}_{n}\left(\sigma, v_{*}\right)-\tilde{H}_{n}\left(\sigma_{*}, v_{*}\right)\right)\right| \rightarrow^{p} 0$ for $0 \leq j \leq 3$. If further (B4) is satisfied, we have

$$
\begin{aligned}
& E_{\Pi}\left[\left(b_{n}^{-1 / 2} \sup _{\sigma}\left|\partial_{\sigma}^{j}\left(H_{n}\left(\sigma, v_{*}\right)-H_{n}\left(\sigma_{*}, v_{*}\right)\right)-\partial_{\sigma}^{j}\left(\tilde{H}_{n}\left(\sigma, v_{*}\right)-\tilde{H}_{n}\left(\sigma_{*}, v_{*}\right)\right)\right|\right)^{q}\right] \\
& \quad=\bar{R}_{n}\left(\left(b_{n}^{-5} k_{n}^{7}\right)^{q / 4}\right)
\end{aligned}
$$

for $0 \leq j \leq 3$.

Taylor's formula yields

$$
\begin{aligned}
& H_{n}\left(\sigma, \hat{v}_{n}\right)-H_{n}\left(\sigma_{*}, \hat{v}_{n}\right)-\left(H_{n}\left(\sigma, v_{*}\right)-H_{n}\left(\sigma_{*}, v_{*}\right)\right) \\
& =-\frac{1}{2} \sum_{m}\left\{Z_{m}^{\top}\left(\tilde{\mathbf{C}}\left(\hat{X}_{m-1}, \hat{v}_{n}\right)-\tilde{\mathbf{C}}\left(\hat{X}_{m-1}, v_{*}\right)\right) Z_{m}+\log \frac{\operatorname{det} S_{m}\left(\sigma, \hat{v}_{n}\right)}{\operatorname{det} S_{m}\left(\sigma, v_{*}\right)}-\log \frac{\operatorname{det} S_{m}\left(\sigma_{*}, \hat{v}_{n}\right)}{\operatorname{det} S_{m}\left(\sigma_{*}, v_{*}\right)}\right\} \\
& =-\frac{1}{2} \sum_{m} \sum_{j=1}^{3}\left\{Z_{m}^{\top} \partial_{v}^{j} \tilde{\mathbf{C}}\left(\hat{X}_{m-1}, v_{*}\right) Z_{m}+\partial_{v}^{j} \log \frac{\operatorname{det} S_{m}\left(\sigma, v_{*}\right)}{\operatorname{det} S_{m}\left(\sigma_{*}, v_{*}\right)}\right\} \frac{\left(\hat{v}_{n}-v_{*}\right)^{j}}{j !} \\
& \quad-\frac{1}{2} \sum_{m} \int_{0}^{1} \frac{(1-s)^{3}}{6}\left\{Z_{m}^{\top} \partial_{v}^{4} \tilde{\mathbf{C}}\left(\hat{X}_{m-1}, v_{s}\right) Z_{m}+\partial_{v}^{4} \log \frac{\operatorname{det} S_{m}\left(\sigma, v_{s}\right)}{\operatorname{det} S_{m}\left(\sigma_{*}, v_{s}\right)}\right\} d s\left(\hat{v}_{n}-v_{*}\right)^{4},
\end{aligned}
$$

where $v_{s}=s \hat{v}_{n}+(1-s) v_{*}$. 
Then we obtain $b_{n}^{-1 / 4}\left|H_{n}\left(\sigma, \hat{v}_{n}\right)-H_{n}\left(\sigma_{*}, \hat{v}_{n}\right)-\left(H_{n}\left(\sigma, v_{*}\right)-H_{n}\left(\sigma_{*}, v_{*}\right)\right)\right| \rightarrow^{p} 0$, by Lemma $4.3,(\mathrm{~V})$, and the equation $\partial_{v} \log \operatorname{det} S_{m}=\operatorname{tr}\left(\partial_{v} S_{m} S_{m}^{-1}\right)$. Similarly, we obtain $b_{n}^{-1 / 4} \times$ $\left|\partial_{\sigma}^{j} H_{n}\left(\sigma, \hat{v}_{n}\right)-\partial_{\sigma}^{j} H_{n}\left(\sigma, v_{*}\right)\right| \rightarrow^{p} 0$ for $1 \leq j \leq 4$. Sobolev's inequality yields $\sup _{\sigma}\left(b_{n}^{-1 / 4} \times\right.$ $\left.\left|\partial_{\sigma}^{j}\left(H_{n}\left(\sigma, \hat{v}_{n}\right)-H_{n}\left(\sigma_{*}, \hat{v}_{n}\right)\right)-\partial_{\sigma}^{j}\left(H_{n}\left(\sigma, v_{*}\right)-H_{n}\left(\sigma_{*}, v_{*}\right)\right)\right|\right) \rightarrow^{p} 0$ for $0 \leq j \leq 3$ and consequently we obtain $\sup _{\sigma}\left(b_{n}^{-1 / 2}\left|\partial_{\sigma}^{j}\left(H_{n}\left(\sigma, \hat{v}_{n}\right)-H_{n}\left(\sigma_{*}, \hat{v}_{n}\right)\right)-\partial_{\sigma}^{j}\left(\tilde{H}_{n}\left(\sigma, v_{*}\right)-\tilde{H}_{n}\left(\sigma_{*}, v_{*}\right)\right)\right|\right) \rightarrow^{p} 0$ for $0 \leq j \leq 3$.

Moreover, points 1 and 3 of Lemma 4.3 yield $E_{\Pi}\left[\left|b_{n}^{-1 / 4} \partial_{\sigma} \hat{\Psi}_{1, n}\left(\sigma_{*}\right)\right|^{2}\right]=\bar{R}_{n}\left(b_{n}^{-3 / 2} k_{n}^{2}\right) \rightarrow^{p} 0$, $E_{\Pi}\left[\left|b_{n}^{-1 / 4} \partial_{\sigma} \hat{\Psi}_{2, n}\left(\sigma_{*}\right)\right|^{2}\right]=O_{p}\left(b_{n}^{-3 / 4} k_{n} b_{n}^{\varepsilon}\right) \rightarrow^{p} 0$ for sufficiently small $\varepsilon>0$, and consequently $b_{n}^{-1 / 4}\left(\partial_{\sigma} H_{n}\left(\sigma_{*}, \hat{v}_{n}\right)-\partial_{\sigma} \tilde{H}_{n}\left(\sigma_{*}, v_{*}\right)\right) \rightarrow^{p} 0$.

If further (B4) is satisfied, then for any $q>0$, (4.7) yields

$$
\begin{aligned}
& \sup _{\sigma} E_{\Pi}\left[b_{n}^{-q / 2}\left|H_{n}\left(\sigma, \hat{v}_{n}\right)-H_{n}\left(\sigma_{*}, \hat{v}_{n}\right)-\left(H_{n}\left(\sigma, v_{*}\right)-H_{n}\left(\sigma_{*}, v_{*}\right)\right)\right|^{q}\right] \\
& \quad=\bar{R}_{n}\left(b_{n}^{-q / 2} b_{n}^{-q} k_{n}^{2 q} \ell_{n}^{q} b_{n}^{-q / 2}\right)+\bar{R}_{n}\left(b_{n}^{-q / 2}\left(b_{n} k_{n} \ell_{n}\right)^{q} b_{n}^{-2 q}\right)=\bar{R}_{n}\left(b_{n}^{-q} k_{n}^{q}\right),
\end{aligned}
$$

by Lemma 4.3, (V), and the equation $\partial_{v} \log \operatorname{det} S_{m}=\operatorname{tr}\left(\partial_{v} S_{m} S_{m}^{-1}\right)$. Similarly, we obtain $\sup _{\sigma} E_{\Pi}\left[b_{n}^{-q / 2}\left|\partial_{\sigma}^{j} H_{n}\left(\sigma, \hat{v}_{n}\right)-\partial_{\sigma}^{j} H_{n}\left(\sigma, v_{*}\right)\right|^{q}\right]=\bar{R}_{n}\left(b_{n}^{-q} k_{n}^{q}\right)$ for $1 \leq j \leq 4$. Sobolev's inequality yields $E_{\Pi}\left[\sup _{\sigma}\left(b_{n}^{-1 / 2}\left|\partial_{\sigma}^{j}\left(H_{n}\left(\sigma, \hat{v}_{n}\right)-H_{n}\left(\sigma_{*}, \hat{v}_{n}\right)\right)-\partial_{\sigma}^{j}\left(H_{n}\left(\sigma, v_{*}\right)-H_{n}\left(\sigma_{*}, v_{*}\right)\right)\right|\right)^{q}\right]=$ $\bar{R}_{n}\left(b_{n}^{-q} k_{n}^{q}\right)$ for $0 \leq j \leq 3$, which completes the proof.

\section{The limit of the quasi-likelihood function}

We complete the proof of Proposition 2.1 in this section. To do so, it is essential to specify the asymptotic behavior of some functions of approximate covariance matrix $\tilde{S}_{m}$, as seen in (5.2). Unlike previous studies by Gloter and Jacod $[12,13]$, the eigenvalues of the diagonal blocks $\tilde{D}_{1, m}$ and $\tilde{D}_{2, m}$ of $\tilde{S}_{m}$ are not identified because of the irregular sampling, and even the sizes of $\tilde{D}_{1, m}$ and $\tilde{D}_{2, m}$ are different. These problems make it difficult to deduce asymptotic behaviors of the right-hand side of (5.2). To solve these problems, in Lemma 5.1, we approximate $\tilde{D}_{j, m}$ by $\dot{D}_{j, m}$, which is a kind of local averaged versions of $\tilde{D}_{j, m}$ and has similar properties to the covariance matrix of equidistant sampling scheme. Moreover, we can also change the sizes of $\tilde{D}_{j, m}$ using some specific properties of $\dot{D}_{j, m}$. We deal with this in Lemma 5.2, and show convergence of some trace functions that appear in a decomposition of $H_{n}$. The decomposition (4.4) and the nice properties of $p_{i}$ in Lemma 4.1 are essential in the proofs.

Lemma 4.4 yields

$$
\begin{aligned}
b_{n}^{-1 / 2} \partial_{\sigma}^{j}\left(H_{n}\left(\sigma, \hat{v}_{n}\right)-H_{n}\left(\sigma_{*}, \hat{v}_{n}\right)\right) \\
=b_{n}^{-1 / 2} \partial_{\sigma}^{j}\left(\tilde{H}_{n}\left(\sigma, v_{*}\right)-\tilde{H}_{n}\left(\sigma_{*}, v_{*}\right)\right)+o_{p}(1) \\
=-\frac{1}{2} b_{n}^{-\frac{1}{2}} \sum_{m}\left(E_{m}\left[\tilde{Z}_{m}^{\top} \partial_{\sigma}^{j}\left(\tilde{S}_{m}^{-1}-\tilde{S}_{m, *}^{-1}\right) \tilde{Z}_{m}\right]+\partial_{\sigma}^{j} \log \frac{\operatorname{det} \tilde{S}_{m}}{\operatorname{det} \tilde{S}_{m, *}}\right) \\
\quad-\frac{1}{2} b_{n}^{-\frac{1}{2}} \sum_{m} \bar{E}_{m}\left[\tilde{Z}_{m}^{\top} \partial_{\sigma}^{j}\left(\tilde{S}_{m}^{-1}-\tilde{S}_{m, *}^{-1}\right) \tilde{Z}_{m}\right]+o_{p}(1)
\end{aligned}
$$


for $0 \leq j \leq 3$. Together with the relation $E_{m}\left[\tilde{Z}_{m}^{\top} \partial_{\sigma}^{j}\left(\tilde{S}_{m}^{-1}-\tilde{S}_{m, *}^{-1}\right) \tilde{Z}_{m}\right]=\operatorname{tr}\left(\partial_{\sigma}^{j}\left(\tilde{S}_{m}^{-1}-\tilde{S}_{m, *}^{-1}\right) \tilde{S}_{m, *}\right)$, we obtain

$$
\begin{aligned}
& b_{n}^{-\frac{1}{2}} \partial_{\sigma}^{j}\left(H_{n}\left(\sigma, \hat{v}_{n}\right)-H_{n}\left(\sigma_{*}, \hat{v}_{n}\right)\right) \\
& \quad=-\frac{1}{2} b_{n}^{-\frac{1}{2}} \sum_{m} \partial_{\sigma}^{j}\left(\operatorname{tr}\left(\tilde{S}_{m}^{-1} \tilde{S}_{m, *}-\mathcal{E}\right)+\log \frac{\operatorname{det} \tilde{S}_{m}}{\operatorname{det} \tilde{S}_{m, *}}\right)+o_{p}(1),
\end{aligned}
$$

since the residual terms are $o_{p}(1)$ by Lemma 4.3.

We first investigate asymptotics of $\operatorname{tr}\left(\tilde{S}_{m}^{-1} \tilde{S}_{m, *}-\mathcal{E}\right)$. Let $\tilde{L}=\left\{\tilde{b}_{m}^{1} \cdot \tilde{b}_{m}^{2}\left|I_{i, m}^{1} \cap I_{j, m}^{2}\right|\right\}_{i, j}$ and $\tilde{G}=\left\{\left|I_{i, m}^{1} \cap I_{j, m}^{2}\right|\right\}_{i, j}$. Then since

$$
\begin{aligned}
\tilde{S}_{m}^{-1} & =\tilde{D}_{m}^{-1 / 2} \sum_{p=0}^{\infty}(-1)^{p}\left(\begin{array}{cc}
0 & \tilde{D}_{1, m}^{-1 / 2} \tilde{L} \tilde{D}_{2, m}^{-1 / 2} \\
\tilde{D}_{2, m}^{-1 / 2} \tilde{L}^{\top} \tilde{D}_{1, m}^{-1 / 2} & 0
\end{array}\right)^{p} \tilde{D}_{m}^{-1 / 2} \\
& =\sum_{p=0}^{\infty}\left(\begin{array}{cc}
\left(\tilde{D}_{1, m}^{-1} \tilde{L} \tilde{D}_{2, m}^{-1} \tilde{L}^{\top}\right)^{p} \tilde{D}_{1, m}^{-1} & -\tilde{D}_{1, m}^{-1} \tilde{L}\left(\tilde{D}_{2, m}^{-1} \tilde{L}^{\top} \tilde{D}_{1, m}^{-1} \tilde{L}\right)^{p} \tilde{D}_{2, m}^{-1} \\
-\tilde{D}_{2, m}^{-1} \tilde{L}^{\top}\left(\tilde{D}_{1, m}^{-1} \tilde{L} \tilde{D}_{2, m}^{-1} \tilde{L}^{\top}\right)^{p} \tilde{D}_{1, m}^{-1} & \left(\tilde{D}_{2, m}^{-1} \tilde{L}^{\top} \tilde{D}_{1, m}^{-1} \tilde{L}\right)^{p} \tilde{D}_{2, m}^{-1}
\end{array}\right),
\end{aligned}
$$

we have

$$
\begin{aligned}
\operatorname{tr}\left(\tilde{S}_{m}^{-1} \tilde{S}_{m, *}-\mathcal{E}\right)= & \sum_{p=0}^{\infty}\left\{\left(\left|\tilde{b}_{m, *}^{1}\right|^{2}-\left|\tilde{b}_{m}^{1}\right|^{2}\right) \operatorname{tr}\left(\left(\tilde{D}_{1, m}^{-1} \tilde{L}_{2, m}^{-1} \tilde{L}^{\top}\right)^{p} \tilde{D}_{1, m}^{-1} D_{1, m}^{\prime}\right)\right. \\
& +\left(\left|\tilde{b}_{m, *}^{2}\right|^{2}-\left|\tilde{b}_{m}^{2}\right|^{2}\right) \operatorname{tr}\left(\left(\tilde{D}_{2, m}^{-1} \tilde{L}^{\top} \tilde{D}_{1, m}^{-1} \tilde{L}\right)^{p} \tilde{D}_{2, m}^{-1} D_{2, m}^{\prime}\right) \\
& \left.-2\left(\tilde{b}_{m, *}^{1} \cdot \tilde{b}_{m, *}^{2}-\tilde{b}_{m}^{1} \cdot \tilde{b}_{m}^{2}\right) \operatorname{tr}\left(\tilde{D}_{1, m}^{-1} \tilde{L} \tilde{D}_{2, m}^{-1}\left(\tilde{L}^{\top} \tilde{D}_{1, m}^{-1} \tilde{L} \tilde{D}_{2, m}^{-1}\right)^{p} \tilde{G}^{\top}\right)\right\} .
\end{aligned}
$$

Note that $\left\|\left\{\left|I_{i, m}^{1} \cap I_{j, m}^{2}\right|\left|I_{i, m}^{1}\right|^{-1 / 2}\left|I_{j, m}^{2}\right|^{-1 / 2}\right\}_{i j}\right\| \leq 1$ by Lemma 2 in [25].

We will see the limit of each term on the right-hand side of (5.3). Let $\hat{a}_{m}^{j}=a_{s_{m-1}}^{j}$ and $\dot{D}_{j, m}=$ $\left|\tilde{b}_{m}^{j}\right|^{2} b_{n}^{-1}\left(\hat{a}_{m}^{j}\right)^{-1} \mathcal{E}+v_{j, *} M_{j, m}$. It is difficult to calculate the eigenvalues of $\tilde{D}_{j, m}$. However, we can easily calculate the eigenvalues of $\dot{D}_{j, m}$ as seen in Section 4.2, and it has nice properties which are useful when we deduce the limit. Moreover, we can replace $\tilde{D}_{j, m}^{-1}$ by $\dot{D}_{j, m}^{-1}$ using the following lemma.

Lemma 5.1. Let $j \in\{1,2\}$ and $A_{n, m}$ be a $k_{m}^{j} \times k_{m}^{j}$ matrix for $1 \leq m \leq \ell_{n}$. Assume (B1), (A2) and that all elements of $A_{n, m}$ are nonnegative and $\left\|A_{n, m}\right\| \leq 1$ for any $m$. Then

$$
\operatorname{tr}\left(\partial_{\sigma}^{k} \tilde{D}_{j, m}^{-1} A_{n, m}\right)=\operatorname{tr}\left(\partial_{\sigma}^{k} \dot{D}_{j, m}^{-1} A_{n, m}\right)+\dot{R}_{n}\left(b_{n}^{3 / 2} \ell_{n}^{-1}\right)
$$

for $0 \leq k \leq 3$. If further (B2) is satisfied, then

$$
\sup _{\sigma}\left|\operatorname{tr}\left(\partial_{\sigma}^{k} \tilde{D}_{j, m}^{-1} A_{n, m}\right)-\operatorname{tr}\left(\partial_{\sigma}^{k} \dot{D}_{j, m}^{-1} A_{n, m}\right)\right|=\underline{R}_{n}\left(b_{n}^{3 / 2} \ell_{n}^{-1}\right)
$$

for $0 \leq k \leq 3$. 
Proof. We first consider the case where $k=0$. (4.4), (4.6), and the equation above it yield

$$
\begin{aligned}
\operatorname{tr}\left(\tilde{D}_{j, m}^{-1} A_{n, m}\right) & \\
= & \sum_{p=0}^{\infty} \operatorname{tr}\left(\check{D}_{j, m}^{-1 / 2}\left(\check{D}_{j, m}^{-1 / 2}\left(\check{D}_{j, m}-\tilde{D}_{j, m}\right) \check{D}_{j, m}^{-1 / 2}\right)^{p} \check{D}_{j, m}^{-1 / 2} A_{n, m}\right) \\
= & \sum_{p=0}^{\infty} \frac{1}{v_{j, *}^{p+1}} \sum_{i_{1}, \ldots, i_{p+1}} \frac{1}{p_{i_{1}} \cdots p_{i_{p+1}}\left(\left|\tilde{b}_{m}^{j}\right|^{2} r_{n} v_{j, *}^{-1}\right)} \\
& \times \sum_{\substack{l_{q} \leq i_{q} \wedge i_{q+1} \\
l^{\prime} \leq i_{p+1}, l^{\prime \prime} \leq i_{1}}} \frac{\left(A_{n, m}\right)_{l^{\prime}, l^{\prime \prime}}}{P_{l^{\prime}, l^{\prime \prime}, i_{p+1}, i_{1}}} \prod_{q=1}^{p} \frac{\left(\check{D}_{j, m}-\tilde{D}_{j, m}\right)_{l_{q}, l_{q}}}{P_{l_{q}, l_{q}, i_{q}, i_{q+1}}},
\end{aligned}
$$

where $P_{k_{1}, k_{2}, l_{1}, l_{2}}=\prod_{m_{1} ; k_{1} \leq m_{1} \leq l_{1}-1} p_{m_{1}} \prod_{m_{2} ; k_{2} \leq m_{2} \leq l_{2}-1} p_{m_{2}}\left(\left|\tilde{b}_{m}^{j}\right|^{2} r_{n} v_{j, *}^{-1}\right)$.

Then the nice properties of $p_{i}$ in Lemma 4.1 will lead us to the desired results. Roughly speaking, we have $1 \leq p_{i}\left(\left|\tilde{b}_{m}^{j}\right|^{2} r_{n} v_{j, *}^{-1}\right) \sim 1+C b_{n}^{-1 / 2}$ for sufficiently large $i$. This means that $P_{k_{1}, k_{2}, l_{1}, l_{2}}$ and $P_{k_{1}^{\prime}, k_{2}^{\prime}, l_{1}, l_{2}}$ are asymptotically equivalent if $\left|k_{1}-k_{1}^{\prime}\right|$ and $\left|k_{2}-k_{2}^{\prime}\right|$ are of order less than $b_{n}^{1 / 2}$. Then we can replace $\tilde{D}_{j, m}$ in the right-hand side of (5.4) by $\dot{D}_{j, m}$ since the diagonal elements of both matrices have the same local average. We will verify these rough sketches by the following.

We first see that terms containing small $l_{q}$ can be ignored. Let $\eta$ be the one in (A2), $\eta^{\prime} \in(\eta, 1 / 2), t_{\tilde{l}, m}=s_{m-1}+T \ell_{n}^{-1} b_{n}^{\eta^{\prime}} / k_{n}+T \ell_{n}^{-1}\left(k_{n}-b_{n}^{\eta^{\prime}}\right)\left[\left(k_{n}-b_{n}^{\eta^{\prime}}\right) b_{n}^{-\eta}\right]^{-1} \tilde{l} / k_{n}$ for $0 \leq \tilde{l} \leq$ $\left[\left(k_{n}-b_{n}^{\eta^{\prime}}\right) b_{n}^{-\eta}\right], \mathcal{I}_{m}(\tilde{l})=\left\{l ; I_{l, m}^{j} \subset\left[t_{\tilde{l}-1, m}, t_{\tilde{l}, m}\right)\right\}$, and $\mathcal{E}^{\prime}=\left\{\delta_{i_{1} i_{2}} 1_{\left\{\inf I_{i_{1}, m}^{j}<t_{0}\right\}}\right\}_{1 \leq i_{1}, i_{2} \leq k_{m}^{j}}$. Then the absolute value of summation involving terms with $l_{q}$ satisfying $\inf I_{l_{q}, m}^{j}<t_{0}$ for some $1 \leq q \leq p$ on the right-hand side of (5.4) is less than

$$
\begin{aligned}
& \left\|A_{n, m}\right\| \sum_{p=1}^{\infty} p\left\|\check{D}_{j, m}^{-1 / 2}\left(\check{D}_{j, m}-\tilde{D}_{j, m}\right) \check{D}_{j, m}^{-1 / 2}\right\|^{p-1}\left\|\check{D}_{j, m}^{-1 / 2}\right\|^{2}\left|\tilde{b}_{m}^{j}\right|^{2} \operatorname{tr}\left(\check{D}_{j, m}^{-1 / 2}\left(r_{n}-\underline{\mathrm{r}}_{n}\right) \mathcal{E}^{\prime} \check{D}_{j, m}^{-1 / 2}\right) \\
& \leq r_{n}^{2} \underline{\mathrm{r}}_{n}^{-2} \operatorname{tr}\left(\check{D}_{j, m}^{-1} \mathcal{E}^{\prime}\right)
\end{aligned}
$$

by (4.6), Lemma A.1, and the assumptions. Let $J_{1}=\left[T \ell_{n}^{-1} b_{n}^{\eta^{\prime}} k_{n}^{-1} r_{n}^{-1}\right]$ and $J_{2}=\left[T \ell_{n}^{-1} b_{n}^{\eta^{\prime}} \times\right.$ $\left.k_{n}^{-1} \underline{\mathrm{r}}_{n}^{-1}\right]+1$, then we obtain $J_{1} \leq \#\left\{i ; \inf I_{i, m}^{j}<t_{0}\right\} \leq J_{2}$. Therefore, point 2 of Lemma 4.1 ensures that $\operatorname{tr}\left(\check{D}_{j, m}^{-1} \mathcal{E}^{\prime}\right)$ is less than

$$
\frac{J_{2}}{\left[k_{m}^{j} / 2\right]-J_{2}} \operatorname{tr}\left(\check{D}_{j, m}^{-1}\right) \leq \frac{2 J_{2}}{\left[k_{m}^{j} / 2\right]} \operatorname{tr}\left(\check{D}_{j, m}^{-1}\right)
$$


if $\left[k_{m}^{j} / 2\right] \geq 2 J_{2}$, and hence the right-hand side of (5.5) is $\bar{R}_{n}\left(\mathcal{V}_{n}\right)$ by $\operatorname{tr}\left(\check{D}_{j, m}^{-1}\right)=\bar{R}_{n}\left(r_{n}^{-1 / 2} k_{n}\right)$, where $\mathcal{V}_{n}=b_{n}^{1 / 2+\eta^{\prime}} 1_{\left\{\left[\underline{k}_{n} / 2\right] \geq 2 J_{2}\right\}}+b_{n}^{1 / 2} k_{n} 1_{\left\{\left[\underline{k}_{n} / 2\right]<2 J_{2}\right\}}$.

On the other hand, for $\tilde{l}, i_{q}, i_{q+1}$ and $l_{q}$ satisfying $l_{q} \in \mathcal{I}_{m}(\tilde{l})$ and $\max \mathcal{I}_{m}(\tilde{l}) \leq i_{q} \wedge i_{q+1}$, Lemma 4.1 yields that $P_{\max } \mathcal{I}_{m}(\tilde{l}), \max \mathcal{I}_{m}(\tilde{l}), i_{q}, i_{q+1} / P_{l_{q}, l_{q}, i_{q}, i_{q+1}}$ is less than 1 and greater than

$$
\left(1+1 / J_{1}+\left|\tilde{b}_{m}^{j}\right|^{2} r_{n} v_{j, *}^{-1} J_{1}\right)^{-C b_{n}^{-1+\eta} \underline{r}_{n}^{-1}} \geq 1-\bar{R}_{n}\left(b_{n}^{\eta-\eta^{\prime}}+b_{n}^{-1+\eta^{\prime}+\eta}\right)
$$

for sufficiently large $n$. Moreover, $\max _{\tilde{l}}\left|\sum_{l \in \mathcal{I}_{m}(\tilde{l})}\left(\tilde{D}_{j, m}-\dot{D}_{j, m}\right)_{l, l}\right|$ is $\dot{R}_{n}\left(b_{n}^{-1+\eta}\right)$ by (A2). We also have $\sup _{\sigma, m} \max _{\tilde{l}}\left|\sum_{l \in \mathcal{I}_{m}(\tilde{l})}\left(\tilde{D}_{j, m}-\dot{D}_{j, m}\right)_{l, l}\right|=\underline{\mathrm{R}}_{n}\left(b_{n}^{-1+\eta}\right)$ if (B2) is satisfied.

Therefore, we obtain

$$
\begin{aligned}
\operatorname{tr} & \left.\tilde{D}_{j, m}^{-1} A_{n, m}\right) \\
= & \sum_{p=0}^{\infty} \frac{1}{v_{j, *}^{p+1}} \sum_{i_{1}, \ldots, i_{p+1}} \frac{1}{p_{i_{1}} \cdots p_{i_{p+1}}\left(\left|\tilde{b}_{m}^{j}\right|^{2} r_{n} v_{j, *}^{-1}\right)} \\
& \times \sum_{l_{q} \leq i_{q} \wedge i_{q+1}, \inf I_{l_{q}, m}^{j} \geq t_{0}} \frac{\left(A_{n, m}\right)_{l^{\prime}, l^{\prime \prime}}}{P_{l^{\prime}, l^{\prime \prime}, i_{p+1}, i_{1}}} \prod_{q=1}^{p} \frac{\left(\check{D}_{j, m}-\tilde{D}_{j, m}\right)_{l_{q}, l_{q}}}{P_{l_{q}, l_{q}, i_{q}, i_{q+1}}} \\
& +\bar{R}_{n}\left(\mathcal{V}_{n}\right) \\
= & \sum_{p=0}^{\infty} \frac{\mathcal{T}_{m, 1}^{n, p}}{v_{j, *}^{p+1}} \sum_{i_{1}, \ldots, i_{p+1}} \frac{1}{p_{i_{1}} \cdots i_{p+1}, l^{\prime \prime} \leq i_{1}} \sum_{p_{i_{p+1}}\left(\left|\tilde{b}_{m}^{j}\right|^{2} r_{n} v_{j, *}^{-1}\right)} \sum_{l^{\prime} \leq i_{p+1}, l^{\prime \prime} \leq i_{1}} \frac{\left(A_{n, m}\right)_{l^{\prime}, l^{\prime \prime}}}{P_{l^{\prime}, l^{\prime \prime}, i_{p+1}, i_{1}}} \\
& \times \prod_{q=1}^{p} \sum_{1 \leq \tilde{l}_{q} \leq\left[\left(k_{n}-b_{n}^{\eta^{\prime}}\right) b_{n}^{-\eta}\right]} \frac{\sum_{l_{q} \in \mathcal{I}_{m}\left(\tilde{l}_{q}\right)}\left(\check{D}_{j, m}-\dot{D}_{j, m}+\mathcal{T}_{m, 3}^{n, p} \mathcal{E}\right)_{l_{q}, l_{q}}}{P_{\max } \mathcal{I}_{m}\left(\tilde{l}_{q}\right), \max \mathcal{I}_{m}\left(\tilde{l}_{q}\right), i_{q}, i_{q+1}} \bar{R}_{n}\left(\mathcal{V}_{n}\right) \\
= & \sum_{p=0}^{\infty} \mathcal{T}_{m, 1}^{n, p}\left(\mathcal{T}_{m, 2}^{n, p}\right) \\
& \left.+\dot{R}_{n}\left(b_{n}^{3 / 2} \ell_{n}^{-1}\right), \check{\operatorname{tr}}_{j, m}^{-1 / 2}\left(\check{D}_{j, m}^{-1 / 2}\left(\check{D}_{j, m}-\dot{D}_{j, m}+\mathcal{T}_{m, 3}^{n, p} \mathcal{E}\right) \check{D}_{j, m}^{-1 / 2}\right)^{p} \check{D}_{j, m}^{-1 / 2} A_{n, m}\right)
\end{aligned}
$$

where $\mathcal{T}_{m, i}^{n, p}$ is a random variable which does not depend on $l_{q}, \tilde{l}_{q}, i_{q}, i_{q+1}$ and satisfies

$$
\left(1-\bar{R}_{n}\left(b_{n}^{\eta-\eta^{\prime}}+b_{n}^{-1+\eta^{\prime}+\eta}\right)\right)^{p} \leq \mathcal{T}_{m, i}^{n, p} \leq 1
$$

for $i=1,2$ and $\sup _{p}\left|\mathcal{T}_{m, 3}^{n, p}\right|=\dot{R}_{n}\left(b_{n}^{-1}\right)$. 
Let $F_{p}(t)=\operatorname{tr}\left(\check{D}_{j, m}^{-1 / 2}\left(\check{D}_{j, m}^{-1 / 2}\left(\check{D}_{j, m}-\dot{D}_{j, m}+t \mathcal{T}_{m, 3}^{n, p} \mathcal{E}\right) \check{D}_{j, m}^{-1 / 2}\right)^{p} \check{D}_{j, m}^{-1 / 2} A_{n, m}\right)$. Then

$$
\begin{aligned}
\left|F_{p}(1)-F_{p}(0)\right| & \leq \int_{0}^{1}\left|F_{p}^{\prime}(t)\right| d t \\
& \leq p\left|\tilde{b}_{m}^{j}\right|^{-2}\left|\mathcal{T}_{m, 3}^{n, p}\right|\left(1-b_{n}^{-1}\left(\hat{a}_{m}^{j}\right)^{-1} r_{n}^{-1}+\left|\tilde{b}_{m}^{j}\right|^{-2} \mathcal{T}_{m, 3}^{n, p} r_{n}^{-1}\right)^{p-1} \bar{R}_{n}\left(r_{n}^{-1 / 2} b_{n}^{2} \ell_{n}^{-1}\right),
\end{aligned}
$$

and hence $\sum_{p=0}^{\infty}\left|\mathcal{T}_{m, 1}^{n, p}\left(\mathcal{T}_{m, 2}^{n, p}\right)^{-1}\right|\left|F_{p}(1)-F_{p}(0)\right| \leq \sup _{p}\left|\mathcal{T}_{m, 3}^{n, p}\right| \cdot \dot{R}_{n}\left(b_{n}^{3 / 2} k_{n}\right)=\dot{R}_{n}\left(b_{n}^{3 / 2} \ell_{n}^{-1}\right)$. Therefore, we obtain the desired conclusion by

$$
\begin{aligned}
& \sum_{p=0}^{\infty} \mid \mathcal{T}_{m, 1}^{n, p}\left(\mathcal{T}_{m, 2}^{n, p}\right)^{-1}-1 \mid F_{p}(0) \\
& \leq C \sum_{p} p \bar{R}_{n}\left(b_{n}^{\eta-\eta^{\prime}}+b_{n}^{-1+\eta+\eta^{\prime}}\right)\left(1+\bar{R}_{n}\left(b_{n}^{\eta-\eta^{\prime}}+b_{n}^{-1+\eta+\eta^{\prime}}\right)\right)^{p-1} \\
& \quad \times\left(1-b_{n}^{-1}\left(\hat{a}_{m}^{j}\right)^{-1} r_{n}^{-1}\right)^{p} r_{n}^{-1 / 2} k_{m}^{j} \\
&=\dot{R}_{n}\left(b_{n}^{3 / 2} \ell_{n}^{-1}\right)
\end{aligned}
$$

For the case $k=1$, we have $\operatorname{tr}\left(\partial_{\sigma} \tilde{D}_{j, m}^{-1} A_{n, m}\right)=-\operatorname{tr}\left(\dot{D}_{j, m}^{-1} \partial_{\sigma} \tilde{D}_{j, m} \dot{D}_{j, m}^{-1} A_{n, m}\right)+\dot{R}_{n}\left(b_{n}^{3 / 2} \ell_{n}^{-1}\right)$ by using the result for $k=0, \partial_{\sigma} \tilde{D}_{j, m}^{-1}=-\tilde{D}_{j, m}^{-1} \partial_{\sigma} \tilde{D}_{j, m} \tilde{D}_{j, m}^{-1},\left\|\tilde{D}_{j, m}^{-1} \partial_{\sigma} \tilde{D}_{j, m}\right\|=\bar{R}_{n}(1)$ and all elements of $\tilde{D}_{j, m}^{-1}$ are nonnegative by a similar argument to (4.4). Then a similar argument to (5.6) enables us to replace $\partial_{\sigma} \tilde{D}_{j, m}$ by $\partial_{\sigma} \dot{D}_{j, m}$. Similarly, we obtain $\operatorname{tr}\left(\partial_{\sigma}^{k} \tilde{D}_{j, m}^{-1} A_{n, m}\right)=$ $\operatorname{tr}\left(\partial_{\sigma}^{k} \dot{D}_{j, m}^{-1} A_{n, m}\right)+\dot{R}_{n}\left(b_{n}^{3 / 2} \ell_{n}^{-1}\right)$ for $k=2,3$.

If further (B2) is satisfied, then similarly we have $\sup _{\sigma}\left|\operatorname{tr}\left(\partial_{\sigma}^{k} \tilde{D}_{j, m}^{-1} A_{n, m}\right)-\operatorname{tr}\left(\partial_{\sigma}^{k} \dot{D}_{j, m}^{-1} A_{n, m}\right)\right|=$ $\underline{\mathrm{R}}_{n}\left(b_{n}^{3 / 2} \ell_{n}^{-1}\right)$.

Remark 5.1. The proof shows that there are upperbounds of the absolute values of residual terms in the statement of Lemma 5.1 which do not depend on $A_{n, m}$.

Let $c_{j}=\left|\tilde{b}_{m}^{j}\right|^{2} b_{n}^{-1}\left(\hat{a}_{m}^{j}\right)^{-1} / v_{j, *}$ and $c_{j}^{\prime}=c_{j}\left(\hat{a}_{m}^{j} / \hat{a}_{m}^{3-j}\right)^{2}$ for $j=1,2$.

Lemma 5.2. Assume (B1) and (A2). Then

$$
\begin{aligned}
& \sup _{\sigma, m}\left|\partial_{\sigma}^{k} \operatorname{tr}\left(\left(\tilde{D}_{1, m}^{-1} \tilde{G} \tilde{D}_{2, m}^{-1} \tilde{G}^{\top}\right)^{p} \tilde{D}_{1, m}^{-1} D_{1, m}^{\prime}\right)-\frac{T \hat{a}_{m}^{1} k_{n}}{\pi} \frac{\left(\hat{a}_{m}^{2}\right)^{p} \partial_{\sigma}^{k} I_{p+1, p}\left(c_{1}, c_{2}^{\prime}\right)}{b_{n}^{2 p+1}\left(\hat{a}_{m}^{1}\right)^{3 p+1} v_{1, *}^{p+1} v_{2, *}^{p}}\right| \\
& \quad=o_{p}\left(b_{n}^{1 / 2} \ell_{n}^{-1}\right), \\
& \sup _{\sigma, m}\left|\partial_{\sigma}^{k} \operatorname{tr}\left(\left(\tilde{D}_{2, m}^{-1} \tilde{G}^{\top} \tilde{D}_{1, m}^{-1} \tilde{G}\right)^{p} \tilde{D}_{2, m}^{-1} D_{2, m}^{\prime}\right)-\frac{T \hat{a}_{m}^{1} k_{n}}{\pi} \frac{\left(\hat{a}_{m}^{2}\right)^{p+1} \partial_{\sigma}^{k} I_{p, p+1}\left(c_{1}, c_{2}^{\prime}\right)}{b_{n}^{2 p+1}\left(\hat{a}_{m}^{1}\right)^{3 p+2} v_{1, *}^{p} v_{2, *}^{p+1}}\right| \\
& \quad=o_{p}\left(b_{n}^{1 / 2} \ell_{n}^{-1}\right),
\end{aligned}
$$




$$
\begin{aligned}
& \sup _{\sigma, m}\left|\partial_{\sigma}^{k} \operatorname{tr}\left(\left(\tilde{D}_{1, m}^{-1} \tilde{G} \tilde{D}_{2, m}^{-1} \tilde{G}^{\top}\right)^{p+1}\right)-\frac{T \hat{a}_{m}^{1} k_{n}}{\pi} \frac{\left(\hat{a}_{m}^{2}\right)^{p+1} \partial_{\sigma}^{k} I_{p+1, p+1}\left(c_{1}, c_{2}^{\prime}\right)}{\left(b_{n}^{2}\left(\hat{a}_{m}^{1}\right)^{3} v_{1, *} v_{2, *}\right)^{p+1}}\right| \\
& \quad=o_{p}\left(b_{n}^{1 / 2} \ell_{n}^{-1}\right)
\end{aligned}
$$

for $0 \leq k \leq 3$ and $p \in \mathbb{Z}_{+}$. If further (B2) is satisfied, then $o_{p}\left(b_{n}^{1 / 2} \ell_{n}^{-1}\right)$ in (5.7)-(5.9) can be replaced by $\underline{R}_{n}\left(b_{n}^{1 / 2} \ell_{n}^{-1}\right)$.

Proof. For any $p \in \mathbb{N}$, Lemma 5.1 yields

$$
b_{n}^{-1 / 2} \partial_{\sigma}^{k} \operatorname{tr}\left(\left(\tilde{D}_{1, m}^{-1} \tilde{G} \tilde{D}_{2, m}^{-1} \tilde{G}^{\top}\right)^{p}\right)=b_{n}^{-1 / 2} \partial_{\sigma}^{k} \operatorname{tr}\left(\left(\dot{D}_{1, m}^{-1} \tilde{G}_{\dot{D}_{2, m}^{-1}}^{-\tilde{G}^{\top}}\right)^{p}\right)+\dot{R}_{n}\left(\ell_{n}^{-1}\right) .
$$

Moreover, we have

$$
\begin{aligned}
& b_{n}^{-1 / 2} \operatorname{tr}\left(\left(\dot{D}_{1, m}^{-1} \tilde{G} \dot{D}_{2, m}^{-1} \tilde{G}^{\top}\right)^{p}\right) \\
& =b_{n}^{-1 / 2} \frac{1}{v_{1, *}^{p} v_{2, *}^{p}} \sum_{\substack{i_{1}, \ldots, i_{p} \\
j_{1}, \ldots, j_{p} \alpha_{2 q} \leq i_{q+1}, \beta_{2 q} \leq j_{q}(1 \leq q \leq p)}} \sum_{\substack{\alpha_{2 q-1} \leq i_{q}, \beta_{2 q-1} \leq j_{q} \\
q=1}}^{p} \frac{\tilde{G}_{\alpha_{2 q-1}, \beta_{2 q-1}}}{\prod_{\alpha_{2 q-1}, \beta_{2 q-1}, i_{q}+1, j_{q}+1}} \frac{\tilde{G}_{\alpha_{2 q}, \beta_{2 q}}}{\tilde{P}_{\alpha_{2 q}, \beta_{2 q}, i_{q+1}, j_{q}}}
\end{aligned}
$$

by (4.4), where $i_{p+1}=i_{1}$ and $\tilde{P}_{\alpha, \beta, i, j}=\prod_{k_{1}=\alpha}^{i-1} p_{k_{1}}\left(c_{1}\right) \prod_{k_{2}=\beta}^{j-1} p_{k_{2}}\left(c_{2}\right)$.

We will apply (4.3) to obtain the limit of the traces. To do so, we need to change the size of matrices $\tilde{G}$ and $\dot{D}_{2, m}^{-1}$. This is again achieved by the nice properties of $p_{i}$. The essential idea is that point 3 of Lemma 4.1 ensures $p_{i} \approx p_{+}$for sufficiently large $i$, and therefore $\tilde{P}_{\alpha, \beta, i, j} \approx p_{+}\left(c_{1}\right)^{i-\alpha} p_{+}\left(c_{2}\right)^{j-\beta} \approx \exp \left(\sqrt{c_{1}}(i-\alpha)+\sqrt{c_{2}}(j-\beta)\right) \approx \dot{P}_{k \alpha, k \beta, k i, k j}$, where $k \in \mathbb{N}$ and $\dot{P}_{\alpha^{\prime}, \beta^{\prime}, i^{\prime}, j^{\prime}}=\prod_{k_{1}=\alpha^{\prime}}^{i^{\prime}-1} p_{k_{1}}\left(c_{1} / k^{2}\right) \prod_{k_{2}=\beta^{\prime}}^{j^{\prime}-1} p_{k_{2}}\left(c_{2} / k^{2}\right)$. The size of $\dot{D}_{2, m}^{-1}$ decides the ranges of summation of $j_{1}, \ldots, j_{p}$ in (5.11). By changing these ranges using the above relation on $\tilde{P}_{\alpha, \beta, i, j}$ and $\dot{P}_{k \alpha, k \beta, k i, k j}$, we can change the size of matrices $\tilde{G}$ and $\dot{D}_{2, m}^{-1}$.

Now we verify the above idea. First, we see that the terms involving small $\alpha_{q}$ or $\beta_{q}$ in (5.11) can be ignored. Let $\eta \in(0,1 / 2)$ be the one in (A2), $\delta \in(1 / 2,1)$ such that $b_{n}^{\delta+\varepsilon} k_{n}^{-1} \rightarrow 0$ for some $\varepsilon>0, \tilde{s}_{l^{\prime}}=s_{m-1}+T \ell_{n}^{-1}\left[k_{n} b_{n}^{-\eta}\right]^{-1}\left(\left(l^{\prime}+\left[b_{n}^{\delta-\eta}\right]\right) \wedge\left[k_{n} b_{n}^{-\eta}\right]\right)$ for $0 \leq l^{\prime} \leq\left(\left[k_{n} b_{n}^{-\eta}\right]-\right.$ $\left.\left[b_{n}^{\delta-\eta}\right]\right) \vee 0, \dot{D}_{3, m}=\left(c_{1} \wedge c_{2}\right)\left(v_{1, *} \wedge v_{2, *}\right) \mathcal{E}_{k_{m}^{1} \vee k_{m}^{2}}+\left(v_{1, *} \wedge v_{2, *}\right) M\left(k_{m}^{1} \vee k_{m}^{2}\right), G^{\prime}=\left\{\mid I_{i, m}^{1} \cap\right.$ $\left.I_{j, m}^{2} \mid 1_{\left\{\inf I_{i, m}^{1} \wedge \inf I_{j, m}^{2}<\tilde{s}_{0}\right\}}\right\}_{1 \leq i, j \leq k_{m}^{1} \vee k_{m}^{2}}, \hat{G}=\left\{\left|I_{i, m}^{1} \cap I_{j, m}^{2}\right| 1_{\left\{i \leq k_{m}^{1} \text { and } j \leq k_{m}^{2}\right\}}\right\}_{1 \leq i, j \leq k_{m}^{1} \vee k_{m}^{2}}$, and $\mathcal{E}^{\prime \prime}=$ $\left\{\delta_{i j} 1_{\left\{\inf I_{i, m}^{1} \wedge \inf I_{i, m}^{2}<\tilde{s}_{0}\right\}}\right\}_{1 \leq i, j \leq k_{m}^{1} \vee k_{m}^{2}}$. Similarly to the proof of Lemma 5.1, the absolute value $\Lambda_{1}$ of a summation involving the terms with $\left(\alpha_{q}, \beta_{q}\right)$ satisfying $\inf I_{\alpha_{q}, m}^{1} \wedge \inf I_{\beta_{q}, m}^{2}<\tilde{s}_{0}$ is less than $p b_{n}^{-1 / 2} \operatorname{tr}\left(\dot{D}_{3, m}^{-1}\left(G^{\prime} \dot{D}_{3, m}^{-1} \hat{G}^{\top}+\hat{G} \dot{D}_{3, m}^{-1}\left(G^{\prime}\right)^{\top}\right)\left(\dot{D}_{3, m}^{-1} \hat{G} \dot{D}_{3, m}^{-1} \hat{G}^{\top}\right)^{p-1}\right)$. Lemma 3 in [25] implies $\left\|G^{\prime}+\left(G^{\prime}\right)^{\top}\right\| \leq 2 r_{n}$ and hence all the eigenvalues of $G^{\prime}+\left(G^{\prime}\right)^{\top}$ are greater than or equal to $-2 r_{n}$. Therefore, $G^{\prime}+\left(G^{\prime}\right)^{\top}+2 r_{n} \mathcal{E}^{\prime \prime}$ is nonnegative definite, and hence Lemma A.1 yields

$$
\begin{aligned}
\Lambda_{1} & \leq 2 p\left(r_{n} / \underline{r}_{n}\right)^{2 p-1} b_{n}^{-1 / 2} \operatorname{tr}\left(\dot{D}_{3, m}^{-1}\left(G^{\prime}+\left(G^{\prime}\right)^{\top}+2 r_{n} \mathcal{E}^{\prime \prime}\right)\right) \\
& \leq 4 p\left(r_{n} / \underline{r}_{n}\right)^{2 p-1} b_{n}^{-1 / 2}\left(\operatorname{tr}\left(\dot{D}_{3, m}^{-1} G^{\prime}\right)+r_{n} \operatorname{tr}\left(\dot{D}_{3, m}^{-1} \mathcal{E}^{\prime \prime}\right)\right) .
\end{aligned}
$$


Let $(\dot{G})_{i, j}=\left(\sum_{l \leq i} G_{l, i}^{\prime}+\sum_{m<i} G_{i, m}^{\prime}\right) \delta_{i j}$ and $\dot{k}=\max \left\{i ; \dot{G}_{i i}>0\right\}$. Then Lemma 4.1 yields

$$
\begin{aligned}
\operatorname{tr}\left(\dot{D}_{3, m}^{-1} G^{\prime}\right) & =\frac{1}{v_{1, *} \wedge v_{2, *}} \sum_{i} \sum_{\alpha, \beta \leq i} \frac{G_{\alpha, \beta}^{\prime}}{p_{\alpha} \cdots p_{i} p_{\beta} \cdots p_{i-1}} \\
& \leq \frac{1}{v_{1, *} \wedge v_{2, *}} \sum_{i} \sum_{\alpha \leq i} \frac{\dot{G}_{\alpha, \alpha}}{p_{\alpha} \cdots p_{i} p_{\alpha} \cdots p_{i-1}} \\
& =\operatorname{tr}\left(\dot{D}_{3, m}^{-1} \dot{G}\right) \leq\left(\dot{D}_{3, m}^{-1}\right)_{\dot{k}, \dot{k}}\left(\tilde{s}_{0}-s_{m-1}+r_{n}\right) \leq \frac{C b_{n}^{-1+\delta}+r_{n}}{\left[\left(k_{m}^{1} \vee k_{m}^{2}\right) / 2\right]-\dot{k}} \operatorname{tr}\left(\dot{D}_{3, m}^{-1}\right)
\end{aligned}
$$

Therefore, we obtain $\Lambda_{1}=\dot{R}_{n}\left(\ell_{n}^{-1}\right)$, and $\Lambda_{1}=\underline{\mathrm{R}}_{n}\left(\ell_{n}^{-1}\right)$ if (B2) is satisfied.

Let $\ddot{D}_{2, m}=v_{2, *} c_{2}\left(\hat{a}_{m}^{2} / \hat{a}_{m}^{1}\right)^{2} \mathcal{E}+v_{2, *} M_{1, m}, i\left(\alpha^{\prime}\right)=\min \left\{i ; S_{i}^{n, 1} \geq \tilde{s}_{\alpha^{\prime}-1}\right\}$, and $j\left(\alpha^{\prime}\right)=$ $\min \left\{j ; S_{j}^{n, 2} \geq \tilde{s}_{\alpha^{\prime}-1}\right\}$. We will show that $b_{n}^{-1 / 2} \operatorname{tr}\left(\left(\dot{D}_{1, m}^{-1} \tilde{G} \dot{D}_{2, m}^{-1} \tilde{G}^{\top}\right)^{p}\right)$ is approximated by $b_{n}^{-1 / 2-2 p}\left(\hat{a}_{m}^{2}\right)^{p}\left(\hat{a}_{m}^{1}\right)^{-3 p} \operatorname{tr}\left(\left(\dot{D}_{1, m}^{-1} \ddot{D}_{2, m}\right)^{p}\right)$. First, a similar argument to the proof of Lemma 5.1 yields $\left|\tilde{P}_{\alpha, \beta, i, j} / \tilde{P}_{i\left(\alpha^{\prime}\right), j\left(\alpha^{\prime}\right), i\left(i^{\prime}\right), j\left(j^{\prime}\right)}-1\right|=\dot{R}_{n}(1)$ for $i\left(\alpha^{\prime}\right) \leq \alpha<i\left(\alpha^{\prime}+1\right), j\left(\alpha^{\prime}\right) \leq \beta<$ $j\left(\alpha^{\prime}+1\right), i\left(i^{\prime}\right) \leq i<i\left(i^{\prime}+1\right)$, and $j\left(j^{\prime}\right) \leq j<j\left(j^{\prime}+1\right)$. Therefore, repeated use of (A2) yields

$$
\begin{aligned}
b_{n}^{-1 / 2} & \operatorname{tr}\left(\left(\dot{D}_{1, m}^{-1} \tilde{G}_{2, m}^{-1} \tilde{G}^{\top}\right)^{p}\right) \\
= & b_{n}^{-1 / 2} \frac{\mathcal{T}_{m, 4}^{n, p}}{v_{1, *}^{p} v_{2, *}^{p}} \\
& \times \sum_{i_{1}^{\prime}, \ldots, i_{p}^{\prime} \alpha_{1}^{\prime}, \ldots, \alpha_{2 p}^{\prime}} \prod_{q=1}^{p} \frac{\left(T b_{n}^{-1+\eta}\right)^{2} \#\left\{i_{q} ; I_{i_{q}, m}^{1} \subset\left[\tilde{s}_{i^{\prime}-1}, \tilde{s}_{i_{q}^{\prime}}\right)\right\} \#\left\{j_{q} ; I_{j_{q}, m}^{2} \subset\left[\tilde{s}_{j_{q}^{\prime}-1}, \tilde{s}_{j_{q}^{\prime}}\right)\right\}}{\tilde{P}_{i\left(\alpha_{2 q-1}^{\prime}\right), j\left(\alpha_{2 q-1}^{\prime}\right), i\left(i_{q}^{\prime}\right), j\left(j_{q}^{\prime}\right)} \tilde{P}_{i\left(\alpha_{2 q}^{\prime}\right), j\left(\alpha_{2 q}^{\prime}\right), i\left(i_{q+1}^{\prime}\right), j\left(j_{q}^{\prime}\right)}} \\
& +\dot{R}_{n}\left(l_{n}^{-1}\right) \\
= & b_{n}^{-1 / 2} \frac{\mathcal{T}_{m, 5}^{n, p}\left(T b_{n}^{-1+\eta}\right)^{2 p}\left(\hat{a}_{m}^{2}\right)^{p}}{v_{1, *}^{p} v_{2, *}^{p}\left(\hat{a}_{m}^{1}\right)^{p}} \\
& \times \sum^{p} \sum_{i_{1}^{\prime}, \ldots, i_{p}^{\prime} \alpha_{1}^{\prime}, \ldots, \alpha_{2 p}^{\prime} j_{q=1}}^{p} \frac{\#\left\{\alpha ; I_{\alpha, m}^{1} \subset\left[\tilde{s}_{\alpha_{2 q-1}^{\prime}-1}, \tilde{s}_{\alpha_{2 q-1}^{\prime}}\right)\right\} \#\left\{\alpha ; I_{\alpha, m}^{1} \subset\left[\tilde{s}_{\alpha_{2 q}^{\prime}-1}, \tilde{s}_{\alpha_{2 q}^{\prime}}\right)\right\}}{\tilde{P}_{i\left(\alpha_{2 q-1}^{\prime}\right), j\left(\alpha_{2 q-1}^{\prime}\right), i\left(i_{q}^{\prime}\right), j\left(j_{q}^{\prime}\right)} \tilde{P}_{i\left(\alpha_{2 q}^{\prime}\right), j\left(\alpha_{2 q}^{\prime}\right), i\left(i_{q+1}^{\prime}\right), j\left(j_{q}^{\prime}\right)}} \\
& +\dot{R}_{n}\left(l_{n}^{-1}\right),
\end{aligned}
$$

where the summations of $\alpha_{1}^{\prime}, \ldots, \alpha_{2 p}^{\prime}$ are over $1 \leq \alpha_{2 q-1}^{\prime} \leq i_{q}^{\prime} \wedge j_{q}^{\prime}$ and $1 \leq \alpha_{2 q}^{\prime} \leq i_{q+1}^{\prime} \wedge j_{q}^{\prime}$ for $1 \leq q \leq p, i_{p+1}^{\prime}=i_{1}^{\prime}$ and $\mathcal{T}_{m, i}^{n, p}$ is a random variable satisfying $\sup _{\sigma, m}\left|\mathcal{T}_{m, i}^{n, p}-1\right|=\dot{R}_{n}(1)$ for $i=4,5$. 
Since Lemma 4.1 and (A2) yield

$$
\begin{aligned}
& p_{j(\alpha)} \cdots p_{j(\beta)-1}\left(c_{2}\right) \\
& \quad=\left(p_{+}\left(c_{2}\right)\right)^{j(\beta)-j(\alpha)}\left(1+\dot{R}_{n}(1)\right) \\
& \quad=\exp \left(\left(b_{n} \hat{a}_{m}^{2}\left(\tilde{s}_{\beta}-\tilde{s}_{\alpha}\right)+\dot{R}_{n}\left(b_{n}^{1 / 2}\right)\right) \log p_{+}\left(c_{2}\right)\right)\left(1+\dot{R}_{n}(1)\right) \\
& \left.\quad=\exp \left(\hat{a}_{m}^{2}\left(\hat{a}_{m}^{1}\right)^{-1}(i(\beta)-i(\alpha))\right) \log p_{+}\left(c_{2}\right)\right)\left(1+\dot{R}_{n}(1)\right)=p_{i(\alpha)} \cdots p_{i(\beta)-1}\left(c_{2}^{\prime}\right)\left(1+\dot{R}_{n}(1)\right),
\end{aligned}
$$

we may replace $\tilde{P}_{i\left(\alpha_{2 q-1}^{\prime}\right), j\left(\alpha_{2 q-1}^{\prime}\right), i\left(i_{q}^{\prime}\right), j\left(j_{q}^{\prime}\right)}$ and $\tilde{P}_{i\left(\alpha_{2 q}^{\prime}\right), j\left(\alpha_{2 q}^{\prime}\right), i\left(i_{q+1}^{\prime}\right), j\left(j_{q}^{\prime}\right)}$ in the right-hand side of (5.12) by $\hat{P}_{i\left(\alpha_{2 q-1}^{\prime}\right), i\left(\alpha_{2 q-1}^{\prime}\right), i\left(i_{q}^{\prime}\right), i\left(j_{q}^{\prime}\right)}$ and $\hat{P}_{i\left(\alpha_{2 q}^{\prime}\right), i\left(\alpha_{2 q}^{\prime}\right), i\left(i_{q+1}^{\prime}\right), i\left(j_{q}^{\prime}\right)}$, respectively, where $\hat{P}_{\alpha, \beta, i, j}=$ $\prod_{k_{1}=\alpha}^{i-1} p_{k_{1}}\left(c_{1}\right) \prod_{k_{2}=\beta}^{j-1} p_{k_{2}}\left(c_{2}^{\prime}\right)$. Therefore, we obtain

$$
\sup _{\sigma, m}\left|b_{n}^{-1 / 2} \operatorname{tr}\left(\left(\dot{D}_{1, m}^{-1} \tilde{G} \dot{D}_{2, m}^{-1} \tilde{G}^{\top}\right)^{p}\right)-b_{n}^{-1 / 2-2 p}\left(\hat{a}_{m}^{2}\right)^{p}\left(\hat{a}_{m}^{1}\right)^{-3 p} \operatorname{tr}\left(\left(\dot{D}_{1, m}^{-1} \ddot{D}_{2, m}^{-1}\right)^{p}\right)\right|=o_{p}\left(\ell_{n}^{-1}\right),
$$

by a similar argument to (5.12). Since $\partial_{\sigma}^{l} \dot{D}_{j, m}=\partial_{\sigma}^{l} c_{j} v_{j, *} \mathcal{E}$ for $1 \leq l \leq 3$, we similarly obtain

$$
\begin{aligned}
& \sup _{\sigma, m}\left|b_{n}^{-1 / 2} \partial_{\sigma}^{k} \operatorname{tr}\left(\left(\dot{D}_{1, m}^{-1} \tilde{G} \dot{D}_{2, m}^{-1} \tilde{G}^{\top}\right)^{p}\right)-b_{n}^{-1 / 2-2 p}\left(\hat{a}_{m}^{2}\right)^{p}\left(\hat{a}_{m}^{1}\right)^{-3 p} \partial_{\sigma}^{k} \operatorname{tr}\left(\left(\dot{D}_{1, m}^{-1} \ddot{D}_{2, m}^{-1}\right)^{p}\right)\right| \\
& \quad=o_{p}\left(\ell_{n}^{-1}\right) .
\end{aligned}
$$

Then (4.3), (5.10) and (5.13) yield (5.9).

We also have (5.7) and (5.8) by a similar argument.

Similar arguments enable us to replace $o_{p}\left(b_{n}^{1 / 2} \ell_{n}^{-1}\right)$ by $\underline{\mathrm{R}}_{n}\left(b_{n}^{1 / 2} \ell_{n}^{-1}\right)$ in (5.7)-(5.9) if (B2) is satisfied.

Remark 5.2. We can also show that the summations of the left-hand side of (5.7)-(5.9) in $1 \leq p<\infty$ are bounded by $\underline{\mathrm{R}}_{n}\left(b_{n}^{1 / 2} \ell_{n}^{-1}\right) \sum_{p=1}^{\infty} p\left(\tilde{b}_{m}^{1} \cdot \tilde{b}_{m}^{2}\left|\tilde{b}_{m}^{1}\right|^{-1}\left|\tilde{b}_{m}^{2}\right|^{-1}\right)^{2(p-l)_{+}}$for some $l \in \mathbb{N}$ if (B2) is satisfied. To show this, it is sufficient to replace $\tilde{D}_{1, m}^{-1} \tilde{G} \tilde{D}_{2, m}^{-1} \tilde{G}^{\top}$ in the traces with $b_{n}^{-1} \hat{a}_{m}^{2}\left(\hat{a}_{m}^{1}\right)^{-3} \dot{D}_{1, m}^{-1} \ddot{D}_{2, m}^{-1}$ one by one, and estimate residual terms separately on the event $\left\{b_{n}^{-1-\varepsilon^{\prime}} \leq \underline{\mathrm{r}}_{n} \leq r_{n} \leq b_{n}^{-1+\varepsilon^{\prime}}\right\}$ and on its complement for some small $\varepsilon^{\prime}>0$. This fact is used in the proof of Proposition 2.2.

Proof of Proposition 2.1. We first prove the results under the additional condition $\left(\mathrm{A}^{\prime}\right)$.

Since $\left\|\tilde{D}_{1, m}^{-1 / 2} \tilde{G} \tilde{D}_{2, m}^{-1} \tilde{G}^{\top} \tilde{D}_{1, m}^{-1 / 2}\right\| \leq\left|\tilde{b}_{m}^{1}\right|^{-2}\left|\tilde{b}_{m}^{2}\right|^{-2}$ by Lemma 3 in [25], for any $\varepsilon, \delta>0$, there exists $P_{1} \in \mathbb{N}$ such that

$$
\sup _{n} P\left[\sup _{\sigma} b_{n}^{-\frac{1}{2}} \sum_{m} \sum_{p=P+1}^{\infty}\left|\partial_{\sigma}^{k}\left(\left(\tilde{b}_{m}^{1} \cdot \tilde{b}_{m}^{2}\right)^{2 p-1} \operatorname{tr}\left(\left(\tilde{D}_{1, m}^{-1} \tilde{G} \tilde{D}_{2, m}^{-1} \tilde{G}^{\top}\right)^{p}\right)\right)\right| \geq \delta\right]<\varepsilon,
$$




$$
\sup _{n} P\left[\sup _{\sigma} b_{n}^{-\frac{1}{2}} \sum_{m} \sum_{p=P+1}^{\infty}\left|\partial_{\sigma}^{k}\left(\frac{\left(\tilde{b}_{m}^{1} \cdot \tilde{b}_{m}^{2}\right)^{2 p-1} \hat{a}_{m}^{1} k_{n}\left(\hat{a}_{m}^{2}\right)^{p} I_{p, p}\left(c_{1}, c_{2}^{\prime}\right)}{\left(b_{n}^{2}\left(\hat{a}_{m}^{1}\right)^{3} v_{1, *} v_{2, *}\right)^{p}}\right)\right| \geq \delta\right]<\varepsilon
$$

for $P \geq P_{1}$. Together with Lemma 5.2, we obtain that

$$
\begin{aligned}
& \sup _{\sigma} \mid b_{n}^{-1 / 2} \partial_{\sigma}^{k} \sum_{m} \sum_{p=1}^{\infty}\left(\tilde{b}_{m}^{1} \cdot \tilde{b}_{m}^{2}\right)^{2 p-1} \operatorname{tr}\left(\left(\tilde{D}_{1, m}^{-1} \tilde{G} \tilde{D}_{2, m}^{-1} \tilde{G}^{\top}\right)^{p}\right) \\
& \quad-\frac{T \hat{a}_{m}^{1} k_{n}}{\pi b_{n}^{1 / 2}} \partial_{\sigma}^{k} \sum_{m} \sum_{p=1}^{\infty} \frac{\left(\tilde{b}_{m}^{1} \cdot \tilde{b}_{m}^{2}\right)^{2 p-1}\left(\hat{a}_{m}^{2}\right)^{p} I_{p, p}\left(c_{1}, c_{2}^{\prime}\right)}{\left(b_{n}^{2}\left(\hat{a}_{m}^{1}\right)^{3} v_{1, *} v_{2, *}\right)^{p}} \mid \stackrel{p}{\rightarrow} 0 .
\end{aligned}
$$

Let $\dot{a}_{m}^{j}=\tilde{a}_{s_{m-1}}^{j}, \mathfrak{C}_{m}=\left|\tilde{b}_{m}^{1}\right|^{2}\left|\tilde{b}_{m}^{2}\right|^{2}-\left(\tilde{b}_{m}^{1} \cdot \tilde{b}_{m}^{2}\right)^{2}$,

$$
\mathfrak{A}_{t}=\left(\tilde{a}_{t}^{1}\left|b_{t}^{1}\right|^{2}+\tilde{a}_{t}^{2}\left|b_{t}^{2}\right|^{2}+2 \sqrt{\tilde{a}_{t}^{1} \tilde{a}_{t}^{2} \operatorname{det}\left(b_{t} b_{t}^{\top}\right)}\right)^{1 / 2},
$$

and

$$
\begin{aligned}
P_{n} & =\left(c_{1}+c_{2}^{\prime}+2 \sqrt{b_{n}^{-2} \hat{a}_{m}^{2}\left(\hat{a}_{m}^{1}\right)^{-3} v_{1, *}^{-1} v_{2, *}^{-1} \mathfrak{C}_{m}}\right)^{1 / 2} \\
& =b_{n}^{-1 / 2}\left(\hat{a}_{m}^{1}\right)^{-1}\left(\dot{a}_{m}^{1}\left|\tilde{b}_{m}^{1}\right|^{2}+\dot{a}_{m}^{2}\left|\tilde{b}_{m}^{2}\right|^{2}+2 \sqrt{\dot{a}_{m}^{1} \dot{a}_{m}^{2} \mathfrak{C}_{m}}\right)^{1 / 2}
\end{aligned}
$$

Then Lemma A.9 yields

$$
\begin{aligned}
& \frac{T \hat{a}_{m}^{1} k_{n}}{\pi b_{n}^{1 / 2}} \partial_{\sigma}^{k} \sum_{m} \sum_{p=1}^{\infty} \frac{\left(\tilde{b}_{m}^{1} \cdot \tilde{b}_{m}^{2}\right)^{2 p-1}\left(\hat{a}_{m}^{2}\right)^{p} I_{p, p}\left(c_{1}, c_{2}^{\prime}\right)}{\left(b_{n}^{2}\left(\hat{a}_{m}^{1}\right)^{3} v_{1, *} v_{2, *}\right)^{p}} \\
& =\partial_{\sigma}^{k} \sum_{m} \frac{T b_{n}^{1 / 2} \hat{a}_{m}^{1} \ell_{n}^{-1} \frac{\hat{a}_{m}^{2} \tilde{b}_{m}^{1} \cdot \tilde{b}_{m}^{2}}{\left(\hat{a}_{m}^{1}\right)^{3} b_{n}^{2} v_{1, *} v_{2, *}}}{b_{n}^{-1 / 2}\left(\hat{a}_{m}^{1}\right)^{-1} \cdot 2\left(\dot{a}_{m}^{1}\left|\tilde{b}_{m}^{1}\right|^{2}+\dot{a}_{m}^{2}\left|\tilde{b}_{m}^{2}\right|^{2}+2 \sqrt{\dot{a}_{m}^{1} \dot{a}_{m}^{2} \mathfrak{C}_{m}}\right)^{1 / 2} \sqrt{\dot{a}_{m}^{1} \dot{a}_{m}^{2}} \sqrt{\mathfrak{C}_{m}} b_{n}^{-1}\left(\hat{a}_{m}^{1}\right)^{-2}} \\
& \quad+o_{p}(1) \\
& =\partial_{\sigma}^{k} \sum_{m} \frac{T \ell_{n}^{-1} \sqrt{\dot{a}_{m}^{1} \dot{a}_{m}^{2}} \tilde{b}_{m}^{1} \cdot \tilde{b}_{m}^{2}}{2 \sqrt{\mathfrak{C}_{m}}\left(\dot{a}_{m}^{1}\left|\tilde{b}_{m}^{1}\right|^{2}+\dot{a}_{m}^{2}\left|\tilde{b}_{m}^{2}\right|^{2}+2 \sqrt{\dot{a}_{m}^{1} \dot{a}_{m}^{2} \mathfrak{C}_{m}}\right)^{1 / 2}+o_{p}(1)} \\
& =\partial_{\sigma}^{k} \int_{0}^{T} \frac{\sqrt{\tilde{a}_{t}^{1} \tilde{a}_{t}^{2}} b_{t}^{1} \cdot b_{t}^{2}}{2 \sqrt{\operatorname{det}\left(b_{t} b_{t}^{\top}\right)} \mathfrak{A}_{t}} d t+o_{p}(1) .
\end{aligned}
$$


Therefore, we have

$$
\begin{aligned}
& \sup _{\sigma} \mid b_{n}^{-1 / 2} \partial_{\sigma}^{k} \sum_{m} \sum_{p=1}^{\infty}\left(\tilde{b}_{m}^{1} \cdot \tilde{b}_{m}^{2}\right)^{2 p-1} \operatorname{tr}\left(\left(\tilde{D}_{1, m}^{-1} \tilde{G} \tilde{D}_{2, m}^{-1} \tilde{G}^{\top}\right)^{p}\right) \\
& \quad-\partial_{\sigma}^{k} \int_{0}^{T} \frac{\sqrt{\tilde{a}_{t}^{1} \tilde{a}_{t}^{2}} b_{t}^{1} \cdot b_{t}^{2}}{2 \sqrt{\operatorname{det}\left(b_{t} b_{t}^{\top}\right)} \mathfrak{A}_{t}} d t \mid \stackrel{p}{\rightarrow} 0 .
\end{aligned}
$$

Similarly, we obtain

$$
\begin{gathered}
\sup _{\sigma} \mid b_{n}^{-1 / 2} \partial_{\sigma}^{k} \sum_{m} \sum_{p=0}^{\infty} \operatorname{tr}\left(\left(\tilde{D}_{1, m}^{-1} \tilde{L} \tilde{D}_{2, m}^{-1} \tilde{L}^{\top}\right)^{p} \tilde{D}_{1, m}^{-1} D_{1, m}^{\prime}\right) \\
-\partial_{\sigma}^{k} \int_{0}^{T} \frac{\left|b_{t}^{2}\right|^{2} \sqrt{\tilde{a}_{t}^{1} \tilde{a}_{t}^{2}}+\tilde{a}_{t}^{1} \sqrt{\operatorname{det}\left(b_{t} b_{t}^{\top}\right)}}{2 \sqrt{\operatorname{det}\left(b_{t} b_{t}^{\top}\right)} \mathfrak{A}_{t}} d t \mid \stackrel{p}{\rightarrow} 0, \\
\sup _{\sigma} \mid b_{n}^{-1 / 2} \partial_{\sigma}^{k} \sum_{m} \sum_{p=0}^{\infty} \operatorname{tr}\left(\left(\tilde{D}_{2, m}^{-1} \tilde{L}^{\top} \tilde{D}_{1, m}^{-1} \tilde{L}\right)^{p} \tilde{D}_{2, m}^{-1} D_{2, m}^{\prime}\right) \\
-\partial_{\sigma}^{k} \int_{0}^{T} \frac{\left|b_{t}^{1}\right|^{2} \sqrt{\tilde{a}_{t}^{1} \tilde{a}_{t}^{2}}+\tilde{a}_{t}^{2} \sqrt{\operatorname{det}\left(b_{t} b_{t}^{\top}\right)}}{2 \sqrt{\operatorname{det}\left(b_{t} b_{t}^{\top}\right)} \mathfrak{A}_{t}} d t \mid \stackrel{p}{\rightarrow} 0 .
\end{gathered}
$$

Lemmas A.3 and 5.2 and a similar argument yield

$$
\begin{aligned}
\partial_{\sigma}^{k} \log \operatorname{det}\left(\tilde{S}_{m} \tilde{D}_{m}^{-1}\right) & =\partial_{\sigma}^{k} \log \operatorname{det}\left(\mathcal{E}+\left(\begin{array}{cc}
0 & \tilde{D}_{1, m}^{-1 / 2} \tilde{L} \tilde{D}_{2, m}^{-1 / 2} \\
\tilde{D}_{2, m}^{-1 / 2} \tilde{L}^{\top} \tilde{D}_{1, m}^{-1 / 2} & 0
\end{array}\right)\right) \\
& =-\sum_{p=1}^{\infty} \frac{1}{p} \partial_{\sigma}^{k} \operatorname{tr}\left(\left(\tilde{D}_{1, m}^{-1} \tilde{L} \tilde{D}_{2, m}^{-1} \tilde{L}^{\top}\right)^{p}\right) \\
& =-\frac{T \hat{a}_{m}^{1} k_{n}}{\pi} \sum_{p=1}^{\infty} \frac{\left(\hat{a}_{m}^{2}\right)^{p}\left(\tilde{b}_{m}^{1} \cdot \tilde{b}_{m}^{2}\right)^{2 p} I_{p, p}\left(c_{1}, c_{2}^{\prime}\right)}{p\left(\hat{a}_{m}^{1}\right)^{3 p} b_{n}^{2 p} v_{1, *}^{p} v_{2, *}^{p}}+o_{p}\left(b_{n}^{1 / 2} \ell_{n}^{-1}\right) .
\end{aligned}
$$

Then Lemma A.9 yields

$$
\begin{aligned}
& \partial_{\sigma}^{k} \log \operatorname{det}\left(\tilde{S}_{m} \tilde{D}_{m}^{-1}\right) \\
& \quad=-T \hat{a}_{m}^{1} k_{n} \partial_{\sigma}^{k}\left(\sqrt{\frac{\left|\tilde{b}_{m}^{1}\right|^{2}}{b_{n} \hat{a}_{m}^{1} v_{1, *}}}+\sqrt{\frac{\hat{a}_{m}^{2}\left|\tilde{b}_{m}^{2}\right|^{2}}{b_{n}\left(\hat{a}_{m}^{1}\right)^{2} v_{2, *}}}\right.
\end{aligned}
$$




$$
\begin{aligned}
& \left.-\frac{b_{n}^{-1 / 2}}{\hat{a}_{m}^{1}}\left(\dot{a}_{m}^{1}\left|\tilde{b}_{m}^{1}\right|^{2}+\dot{a}_{m}^{2}\left|\tilde{b}_{m}^{2}\right|^{2}+2 \sqrt{\dot{a}_{m}^{1} \dot{a}_{m}^{2} \operatorname{det}\left(\tilde{b}_{m} \tilde{b}_{m}^{\top}\right)}\right)^{1 / 2}\right) \\
& +o_{p}\left(b_{n}^{1 / 2} \ell_{n}^{-1}\right) \\
= & T b_{n}^{1 / 2} \ell_{n}^{-1} \partial_{\sigma}^{k}\left(\left(\dot{a}_{m}^{1}\left|\tilde{b}_{m}^{1}\right|^{2}+\dot{a}_{m}^{2}\left|\tilde{b}_{m}^{2}\right|^{2}+2 \sqrt{\dot{a}_{m}^{1} \dot{a}_{m}^{2} \operatorname{det}\left(\tilde{b}_{m} \tilde{b}_{m}^{\top}\right)}\right)^{1 / 2}-\sqrt{\dot{a}_{m}^{1}\left|\tilde{b}_{m}^{1}\right|^{2}}-\sqrt{\dot{a}_{m}^{2}\left|\tilde{b}_{m}^{2}\right|^{2}}\right) \\
& +o_{p}\left(b_{n}^{1 / 2} \ell_{n}^{-1}\right) .
\end{aligned}
$$

Moreover, Lemmas A.3 and 5.1 yield

$$
\begin{aligned}
\partial_{\sigma}^{k} & \log \operatorname{det}\left(\tilde{D}_{j, m} \tilde{D}_{j, m, *}^{-1}\right) \\
& =\partial_{\sigma}^{k} \log \operatorname{det}\left(\mathcal{E}+\tilde{D}_{j, m, *}^{-1 / 2}\left(\tilde{D}_{j, m}-\tilde{D}_{j, m, *}\right) \tilde{D}_{j, m, *}^{-1 / 2}\right) \\
& =\sum_{p=1}^{\infty} \frac{(-1)^{p-1}}{p} \partial_{\sigma}^{k} \operatorname{tr}\left(\left(\tilde{D}_{j, m, *}^{-1}\left(\tilde{D}_{j, m}-\tilde{D}_{j, m, *}\right)\right)^{p}\right) \\
& =\sum_{p=1}^{\infty} \frac{(-1)^{p-1}}{p} \partial_{\sigma}^{k} \operatorname{tr}\left(\left(\dot{D}_{j, m, *}^{-1}\left(\dot{D}_{j, m}-\dot{D}_{j, m, *}\right)\right)^{p}\right)+o_{p}\left(b_{n}^{1 / 2} \ell_{n}^{-1}\right) \\
& =\partial_{\sigma}^{k} \log \operatorname{det}\left(\dot{D}_{j, m} \dot{D}_{j, m, *}^{-1}\right)+o_{p}\left(b_{n}^{1 / 2} \ell_{n}^{-1}\right)
\end{aligned}
$$

when $\left|\tilde{b}_{m, *}^{j}\right| \geq\left|\tilde{b}_{m}^{j}\right|$, where $\tilde{D}_{j, m, *}$ and $\dot{D}_{j, m, *}$ are obtained by substituting $\sigma=\sigma_{*}$ in $\tilde{D}_{j, m}$ and $\dot{D}_{j, m}$, respectively. Similarly, we have $\partial_{\sigma}^{k} \log \operatorname{det}\left(\tilde{D}_{j, m} \tilde{D}_{j, m, *}^{-1}\right)=\partial_{\sigma}^{k} \log \operatorname{det}\left(\dot{D}_{j, m} \dot{D}_{j, m, *}^{-1}\right)+$ $o_{p}\left(b_{n}^{1 / 2} \ell_{n}^{-1}\right)$ when $\left|\tilde{b}_{m, *}^{j}\right|<\left|\tilde{b}_{m}^{j}\right|$.

On the other hand, results in Section 4.2 yield

$$
\begin{aligned}
\partial_{\sigma}^{k} \log \frac{\operatorname{det} \dot{D}_{j, m}}{\operatorname{det} \dot{D}_{j, m, *}} & =\frac{k_{m}^{j}+1}{\pi} \partial_{\sigma}^{k} \int_{0}^{\pi} \log \frac{c_{j}+2(1-\cos x)}{c_{j, *}+2(1-\cos x)} d x+o_{p}\left(b_{n}^{1 / 2} \ell_{n}^{-1}\right) \\
& =2\left(k_{m}^{j}+1\right) \partial_{\sigma}^{k} \log \frac{\sqrt{c_{j}}+\sqrt{4+c_{j}}}{\sqrt{c_{j, *}}+\sqrt{4+c_{j, *}}}+o_{p}\left(b_{n}^{1 / 2} \ell_{n}^{-1}\right) \\
& =k_{m}^{j} \partial_{\sigma}^{k}\left(\sqrt{c_{j}}-\sqrt{c_{j, *}}\right)+o_{p}\left(b_{n}^{1 / 2} \ell_{n}^{-1}\right) \\
& =T b_{n}^{1 / 2} \ell_{n}^{-1} \sqrt{\dot{a}_{m}^{j}} \partial_{\sigma}^{k}\left(\left|\tilde{b}_{m}^{j}\right|-\left|\tilde{b}_{m, *}^{j}\right|\right)+o_{p}\left(b_{n}^{1 / 2} \ell_{n}^{-1}\right) .
\end{aligned}
$$

The residuals are bounded uniformly with respect to $\sigma$ and $m$. Then we obtain $\sup _{\sigma} \mid b_{n}^{-1 / 2} \times$ $\partial_{\sigma}^{k}\left(H_{n}\left(\sigma, \hat{v}_{n}\right)-H_{n}\left(\sigma_{*}, \hat{v}_{n}\right)\right)-\partial_{\sigma}^{k} \mathcal{Y}_{1}(\sigma) \mid \rightarrow^{p} 0$ as $n \rightarrow \infty$ for any $\sigma \in \Lambda$ and $0 \leq k \leq 3$ by (5.2), (5.3) and (5.16) $-(5.21)$.

Finally, we obtain the desired results without $\left(\mathrm{A} 1^{\prime}\right)$ by using the arguments in Proposition 3.1 of Gloter and Jacod [13]. 


\section{Identifiability of the model}

In this section, we check the identifiability condition, $\inf _{\sigma \neq \sigma_{*}}\left(\left(-\mathcal{Y}_{1}(\sigma)\right) /\left|\sigma-\sigma_{*}\right|^{2}\right)>0$ almost surely. This condition is necessary to deduce consistency of the maximum-likelihood-type estimator, as seen in Proposition 7.1. In general, it is not easy to check this condition directly because $\mathcal{Y}_{1}(\sigma)$ is a complicated function of $b_{t}$ and $a_{t}^{j}$. On the other hand, Ogihara and Yoshida [25] proved that the identifiability condition (A3) of a model for equidistant observations without noise is sufficient for the identifiability of a model for nonsynchronous observations. This is also the case for our model. Recall that $\bar{\rho}$ has been defined just before Lemma 4.2.

Proposition 6.1. Assume (A1), (A2) and (V). Then there exists a positive constant $c$ such that

$$
-\mathcal{Y}_{1}(\sigma) \geq \chi \int_{0}^{T}\left\{\left(\left|b_{t}^{1}\right|^{2}-\left|b_{t, *}^{1}\right|^{2}\right)^{2}+\left(\left|b_{t}^{2}\right|^{2}-\left|b_{t, *}^{2}\right|^{2}\right)^{2}+\left(b_{t}^{1} \cdot b_{t}^{2}-b_{t, *}^{1} \cdot b_{t, *}^{2}\right)^{2}\right\} d t
$$

for any $\sigma$, where

$$
\chi=c\left(1-\bar{\rho}^{2}\right) \frac{\inf _{j, t}\left(a_{t}^{j} / v_{j, *}\right)}{\sup _{j, t}\left(a_{t}^{j} / v_{j, *}\right)^{1 / 2}}\left(\sup _{j, t, \sigma}\left(\left|b^{j}\left(t, X_{t}, \sigma\right)\right| \vee\left|b^{j}\left(t, X_{t}, \sigma\right)\right|^{-1}\right)\right)^{-9} .
$$

In particular, $\inf _{\sigma \neq \sigma_{*}}\left(\left(-\mathcal{Y}_{1}(\sigma)\right) /\left|\sigma-\sigma_{*}\right|^{2}\right)>0$ almost surely under (A1)-(A3) and (V).

Proof. It is sufficient to show the results under the additional condition (A1') by localization techniques similar to the proof of Proposition 2.1.

Let $\tilde{\rho}_{m}=\left|\tilde{b}_{m}^{1}\right|^{-1}\left|\tilde{b}_{m}^{2}\right|^{-1} \tilde{b}_{m}^{1} \cdot \tilde{b}_{m}^{2}, \hat{D}_{m}=\tilde{D}_{m}-M_{m, *}$ and $\mathbf{B}=\sup _{j, t, \sigma}\left(\left|b^{j}\left(t, X_{t}, \sigma\right)\right| \vee\right.$ $\left.\left|b^{j}\left(t, X_{t}, \sigma\right)\right|^{-1}\right)$, then since

$$
\begin{aligned}
& u^{\top} \hat{D}_{m}^{-1 / 2} \tilde{S}_{m} \hat{D}_{m}^{-1 / 2} u \\
& \geq u^{\top}\left(\begin{array}{cc}
\mathcal{E} & \left.\tilde{\rho}_{m}\left\{\left|I_{i, m}^{1} \cap I_{j, m}^{2}\right|\left|I_{i, m}^{1}\right|^{-1 / 2}\left|I_{j, m}^{2}\right|^{-1 / 2}\right\}_{i, j}\right) u \\
\tilde{\rho}_{m}\left\{\left|I_{i, m}^{1} \cap I_{j, m}^{2}\right|\left|I_{i, m}^{1}\right|^{-1 / 2}\left|I_{j, m}^{2}\right|^{-1 / 2}\right\}_{j, i} & \mathcal{E}
\end{array}\right.
\end{aligned}
$$

for any $u \in \mathbb{R}^{k_{m}^{1}+k_{m}^{2}}$, we have $\left\|\left(\hat{D}_{m}^{1 / 2} \tilde{S}_{m}^{-1} \hat{D}_{m}^{1 / 2}\right)^{1 / 2}\right\| \leq C \mathbf{B}\left(1-\bar{\rho}^{2}\right)^{-1 / 2}$ by Lemma A.4, and hence we obtain

$$
\begin{aligned}
& \left\|\left(\hat{D}_{m}^{1 / 2} \tilde{S}_{m}^{-1} \hat{D}_{m}^{1 / 2}\right)^{1 / 2}\left(\hat{D}_{m}^{-1 / 2} \tilde{S}_{m, *} \hat{D}_{m}^{-1 / 2}\right)\left(\hat{D}_{m}^{1 / 2} \tilde{S}_{m}^{-1} \hat{D}_{m}^{1 / 2}\right)^{1 / 2}\right\| \\
& \quad=\left\|\mathcal{E}+\left(\hat{D}_{m}^{1 / 2} \tilde{S}_{m}^{-1} \hat{D}_{m}^{1 / 2}\right)^{1 / 2}\left(\hat{D}_{m}^{-1 / 2}\left(\tilde{S}_{m, *}-\tilde{S}_{m}\right) \hat{D}_{m}^{-1 / 2}\right)\left(\hat{D}_{m}^{1 / 2} \tilde{S}_{m}^{-1} \hat{D}_{m}^{1 / 2}\right)^{1 / 2}\right\| \\
& \quad \leq 1+C \mathbf{B}^{6}\left(1-\bar{\rho}^{-2}\right)^{-1} .
\end{aligned}
$$

Then Lemma A.6 yields

$$
\begin{aligned}
& \operatorname{tr}\left(\tilde{S}_{m}^{-1} \tilde{S}_{m, *}-\mathcal{E}\right)+\log \operatorname{det} \tilde{S}_{m}-\log \operatorname{det} \tilde{S}_{m, *} \\
& \quad \geq C \mathbf{B}^{-6}\left(1-\bar{\rho}^{2}\right) \operatorname{tr}\left(\tilde{S}_{m}^{-1}\left(\tilde{S}_{m, *}-\tilde{S}_{m}\right) \tilde{S}_{m}^{-1}\left(\tilde{S}_{m, *}-\tilde{S}_{m}\right)\right) .
\end{aligned}
$$


Therefore, repeated use of Lemma A.5 yields

$$
\begin{aligned}
& \operatorname{tr}\left(\tilde{S}_{m}^{-1} \tilde{S}_{m, *}-\mathcal{E}\right)+\log \operatorname{det} \tilde{S}_{m}-\log \operatorname{det} \tilde{S}_{m, *} \\
& \geq C \mathbf{B}^{-6}\left(1-\bar{\rho}^{2}\right) \operatorname{tr}\left(\tilde{D}_{m}^{-1}\left(\tilde{S}_{m, *}-\tilde{S}_{m}\right) \tilde{S}_{m}^{-1}\left(\tilde{S}_{m, *}-\tilde{S}_{m}\right)\right) \\
& \geq C \mathbf{B}^{-6}\left(1-\bar{\rho}^{2}\right) \operatorname{tr}\left(\tilde{D}_{m}^{-1}\left(\tilde{S}_{m, *}-\tilde{S}_{m}\right) \tilde{D}_{m}^{-1}\left(\tilde{S}_{m, *}-\tilde{S}_{m}\right)\right) \\
& =C \mathbf{B}^{-6}\left(1-\bar{\rho}^{2}\right)\left\{\sum_{j=1}^{2}\left(\left|\tilde{b}_{m, *}^{j}\right|^{2}-\left|\tilde{b}_{m}^{j}\right|^{2}\right)^{2} \operatorname{tr}\left(\tilde{D}_{j, m}^{-1} D_{j, m}^{\prime} \tilde{D}_{j, m}^{-1} D_{j, m}^{\prime}\right)\right. \\
& \left.\quad+2\left(\tilde{b}_{m, *}^{1} \cdot \tilde{b}_{m, *}^{2}-\tilde{b}_{m}^{1} \cdot \tilde{b}_{m}^{2}\right)^{2} \operatorname{tr}\left(\tilde{D}_{1, m}^{-1} \tilde{G} \tilde{D}_{2, m}^{-1} \tilde{G}^{\top}\right)\right\}
\end{aligned}
$$

Hence, it is sufficient to show that $\limsup$ of $\operatorname{tr}\left(\tilde{D}_{j, m}^{-1} D_{j, m}^{\prime} \tilde{D}_{j, m}^{-1} D_{j, m}^{\prime}\right)$ for $j=1,2$ and $\operatorname{tr}\left(\tilde{D}_{1, m}^{-1} \tilde{G} \tilde{D}_{2, m}^{-1} \tilde{G}^{\top}\right)$ are estimated from below by positive random variables.

By Lemma 5.1, (4.2) and (5.13) with a sampling scheme $\left\{S_{i}^{n, 1}\right\}_{i} \equiv\left\{S_{j}^{n, 2}\right\}_{j}$, we obtain

$$
\begin{aligned}
& b_{n}^{-1 / 2} \operatorname{tr}\left(\tilde{D}_{j, m}^{-1} D_{j, m}^{\prime} \tilde{D}_{j, m}^{-1} D_{j, m}^{\prime}\right) \\
& =b_{n}^{-1 / 2} \operatorname{tr}\left(\dot{D}_{j, m}^{-1} D_{j, m}^{\prime} \dot{D}_{j, m}^{-1} D_{j, m}^{\prime}\right)+\dot{R}_{n}\left(\ell_{n}^{-1}\right)=b_{n}^{-5 / 2}\left(\hat{a}_{m}^{j}\right)^{-2} \operatorname{tr}\left(\dot{D}_{j, m}^{-2}\right)+\dot{R}_{n}\left(\ell_{n}^{-1}\right) \\
& =\frac{b_{n}^{-5 / 2}\left(k_{m}^{j}+1\right)}{\left(\hat{a}_{m}^{j}\right)^{2} v_{j, *}^{2} \pi} I_{2}\left(\frac{b_{n}^{-1}\left|\tilde{b}_{m}^{j}\right|^{2}}{\hat{a}_{m}^{j} v_{j, *}}\right)+\dot{R}_{n}\left(\ell_{n}^{-1}\right)=\frac{T\left(\hat{a}_{m}^{j}\right)^{1 / 2} \ell_{n}^{-1}}{4 v_{j, *}^{1 / 2}\left|\tilde{b}_{m}^{j}\right|^{3}}+\dot{R}_{n}\left(\ell_{n}^{-1}\right) .
\end{aligned}
$$

Moreover, Lemma 5.1, (4.2) and (5.13) yield

$$
\begin{aligned}
& b_{n}^{-1 / 2} \operatorname{tr}\left(\tilde{D}_{1, m}^{-1} \tilde{G} \tilde{D}_{2, m}^{-1} \tilde{G}^{\top}\right) \\
& \quad=b_{n}^{-5 / 2} \frac{\hat{a}_{m}^{2}}{\left(\hat{a}_{m}^{1}\right)^{3}} \operatorname{tr}\left(\dot{D}_{1, m}^{-1} \ddot{D}_{2, m}^{-1}\right)+\dot{R}_{n}\left(\ell_{n}^{-1}\right) \\
& \quad \geq \frac{b_{n}^{-5 / 2} \hat{a}_{m}^{2}}{\left(\hat{a}_{m}^{1}\right)^{3} v_{1, *} v_{2, *}} \operatorname{tr}\left(\left(\left(\left(\frac{\left|\tilde{b}_{m}^{2}\right|^{2} b_{n}^{-1} \hat{a}_{m}^{2}}{v_{2, *}\left(\hat{a}_{m}^{1}\right)^{2}}\right) \vee \frac{\left|\tilde{b}_{m}^{1}\right|^{2} b_{n}^{-1}}{\hat{a}_{m}^{1} v_{1, *}}\right) \mathcal{E}+M_{1, m}\right)^{-2}\right)+\dot{R}_{n}\left(\ell_{n}^{-1}\right) \\
& =\frac{T \ell_{n}^{-1} \dot{a}_{m}^{1} \dot{a}_{m}^{2}}{4\left(\left(\dot{a}_{m}^{1}\left|\tilde{b}_{m}^{1}\right|^{2}\right) \vee\left(\dot{a}_{m}^{2}\left|\tilde{b}_{m}^{2}\right|^{2}\right)\right)^{3 / 2}}+\dot{R}_{n}\left(\ell_{n}^{-1}\right) .
\end{aligned}
$$

Therefore, we obtain (6.1).

In particular, by Lemma 6 and Remark 4 in [25], there exists a positive-valued random variable $\mathcal{R}$ such that

$$
-\mathcal{Y}_{1}(\sigma) \geq \chi \mathcal{R}\left(-\mathcal{Y}_{0}(\sigma)\right)
$$

for any $\sigma$. Therefore, we have $\inf _{\sigma \neq \sigma_{*}}\left(\left(-\mathcal{Y}_{1}(\sigma)\right) /\left|\sigma-\sigma_{*}\right|^{2}\right)>0$ almost surely under (A1)-(A3) and $(\mathrm{V})$. 


\section{Asymptotic mixed normality of the estimator}

In this section, we prove the consistency and asymptotic mixed normality of $\hat{\sigma}_{n}$. To obtain asymptotic mixed normality, we prove stable convergence of the score function $b_{n}^{-1 / 4} \partial_{\sigma} H_{n}\left(\sigma_{*}, v_{*}\right)$ by means of the martingale limit theorem for a mixed normal limit in Jacod [18]. We also use the idea by Jacod et al. [19] to adapt the limit theorem to models containing observation noise.

Consistency is an immediate consequence of Proposition 2.1 and the identifiability condition.

Proposition 7.1. Assume (A1)-(A3) and (V). Then $\hat{\sigma}_{n} \rightarrow^{p} \sigma_{*}$ as $n \rightarrow \infty$.

Proof. Let $\varepsilon, \delta$ be arbitrary positive constants. By Proposition 2.1, we have $\sup _{\sigma} \mid b_{n}^{-1 / 2} \times$ $\left(H_{n}\left(\sigma, \hat{v}_{n}\right)-H_{n}\left(\sigma_{*}, \hat{v}_{n}\right)\right)-\mathcal{Y}_{1}(\sigma) \mid \rightarrow^{p} 0$ as $n \rightarrow \infty$. Moreover, Proposition 6.1 ensures that there exists $\eta>0$ such that $P\left[\inf _{\sigma \neq \sigma_{*}}\left(\left(-\mathcal{Y}_{1}(\sigma)\right) /\left|\sigma-\sigma_{*}\right|^{2}\right) \leq \eta\right]<\varepsilon$. Since $H_{n}\left(\hat{\sigma}_{n}, \hat{v}_{n}\right)-$ $H_{n}\left(\sigma_{*}, \hat{v}_{n}\right) \geq 0$ by the definition of $\hat{\sigma}_{n}$, we obtain

$$
\begin{aligned}
P\left[\left|\hat{\sigma}_{n}-\sigma_{*}\right| \geq \delta\right] & <P\left[\mathcal{Y}_{1}\left(\hat{\sigma}_{n}\right) \leq-\eta \delta^{2}\right]+\varepsilon \\
& \leq P\left[\sup _{\sigma}\left|b_{n}^{-1 / 2}\left(H_{n}\left(\sigma, \hat{v}_{n}\right)-H_{n}\left(\sigma_{*}, \hat{v}_{n}\right)\right)-\mathcal{Y}_{1}(\sigma)\right| \geq \eta \delta^{2}\right]+\varepsilon \\
& <2 \varepsilon
\end{aligned}
$$

for sufficiently large $n$.

Proposition 7.2. Assume (A1), (A2) and (V). Then $b_{n}^{-1 / 4} \partial_{\sigma} H_{n}\left(\sigma_{*}, \hat{v}_{n}\right) \rightarrow^{s-\mathcal{L}} \Gamma_{1}^{1 / 2} \mathcal{N}$ as $n \rightarrow \infty$.

Proof. It is sufficient to prove the results assuming the additional condition (A1').

Since $b_{n}^{-1 / 4} \partial_{\sigma} \tilde{H}_{n}\left(\sigma_{*}, v_{*}\right)=-2^{-1} b_{n}^{-1 / 4} \sum_{m} \bar{E}_{m}\left[\tilde{Z}_{m}^{\top} \partial_{\sigma} \tilde{S}_{m, *}^{-1} \tilde{Z}_{m}\right]$, we only need to check assumptions of Theorem 3.2 in Jacod [18] for $\mathcal{X}_{m}^{n}=-2^{-1} b_{n}^{-1 / 4} \bar{E}_{m}\left[\tilde{Z}_{m}^{\top} \partial_{\sigma} \tilde{S}_{m, *}^{-1} \tilde{Z}_{m}\right]$. For any $\varepsilon>0$, Lemma 4.3 yields

$$
\sum_{m=2}^{\left[\ell_{n} t / T\right]} E_{m}\left[\left|\mathcal{X}_{m}^{n}\right|^{2} 1_{\left\{\left|\mathcal{X}_{m}^{n}\right|>\varepsilon\right\}}\right] \leq \frac{C b_{n}^{-1}}{\varepsilon^{2}} \sum_{m=2}^{\left[\ell_{n} t / T\right]} E_{m}\left[\left(\tilde{Z}_{m}^{\top} \partial_{\sigma} \tilde{S}_{m, *}^{-1} \tilde{Z}_{m}\right)^{4}\right] \stackrel{p}{\rightarrow} 0
$$

It is easy to see that $\sum_{m=2}^{\left[\ell_{n} t / T\right]} E_{m}\left[\mathcal{X}_{m}^{n}\left(W_{s_{m}}-W_{s_{m-1}}\right)\right]=0$.

Lemma 4.3 yields

$$
\begin{aligned}
E_{m}\left[\left(\mathcal{X}_{m}^{n}\right)^{2}\right] & =\frac{b_{n}^{-1 / 2}}{4}\left\{E_{m}\left[\left(\tilde{Z}_{m} \partial_{\sigma} \tilde{S}_{m, *}^{-1} \tilde{Z}_{m}\right)^{2}\right]-E_{m}\left[\tilde{Z}_{m} \partial_{\sigma} \tilde{S}_{m, *}^{-1} \tilde{Z}_{m}\right]^{2}\right\} \\
& =\frac{b_{n}^{-1 / 2}}{2} \operatorname{tr}\left(\tilde{S}_{m, *} \partial_{\sigma} \tilde{S}_{m, *}^{-1} \tilde{S}_{m, *} \partial_{\sigma} \tilde{S}_{m, *}^{-1}\right)+\bar{R}_{n}\left(b_{n}^{-1 / 2}\right) .
\end{aligned}
$$

On the other hand, since $\partial_{\sigma} \log \operatorname{det} \tilde{S}_{m}(x, \sigma)=\operatorname{tr}\left(\partial_{\sigma} \tilde{S}_{m} \tilde{S}_{m}^{-1}\right)$, we have

$$
\begin{aligned}
& \left.E_{m}\left[\tilde{Z}_{m}^{\top} \partial_{\sigma}^{2} \tilde{S}_{m}^{-1} \tilde{Z}_{m}+\partial_{\sigma}^{2} \log \operatorname{det} \tilde{S}_{m}\right]\right|_{\sigma=\sigma_{*}} \\
& \quad=\operatorname{tr}\left(\partial_{\sigma}^{2} \tilde{S}_{m, *}^{-1} \tilde{S}_{m, *}\right)-\operatorname{tr}\left(\partial_{\sigma}^{2} \tilde{S}_{m, *}^{-1} \tilde{S}_{m, *}\right)+\operatorname{tr}\left(\tilde{S}_{m, *}^{-1} \partial_{\sigma} \tilde{S}_{m, *} \tilde{S}_{m, *}^{-1} \partial_{\sigma} \tilde{S}_{m, *}\right) .
\end{aligned}
$$


Therefore, we have

$$
\begin{aligned}
& \sum_{m=2}^{\left[\ell_{n} t / T\right]} E_{m}\left[\left(\mathcal{X}_{m}^{n}\right)^{2}\right] \\
& \left.\quad=\frac{b_{n}^{-1 / 2}}{2} \sum_{m=2}^{\left[\ell_{n} t / T\right]} E_{m}\left[\tilde{Z}_{m}^{\top} \partial_{\sigma}^{2} \tilde{S}_{m}^{-1} \tilde{Z}_{m}+\partial_{\sigma}^{2} \log \operatorname{det} \tilde{S}_{m}\right)\right]\left.\right|_{\sigma=\sigma_{*}} \stackrel{p}{\rightarrow}-\partial_{\sigma}^{2} \mathcal{Y}_{1}\left(\sigma_{*}, t\right),
\end{aligned}
$$

where

$$
\begin{aligned}
& \mathcal{Y}_{1}(\sigma, t) \\
& =\int_{0}^{t}\left\{\frac{\sum_{j=1}^{2}\left(\left|b_{s}^{j}\right|^{2}-\left|b_{s, *}^{j}\right|^{2}\right)\left(\left|b_{s}^{3-j}\right|^{2} \sqrt{\tilde{a}_{s}^{1} \tilde{a}_{s}^{2}}+\tilde{a}_{s}^{j} \sqrt{\operatorname{det}\left(b_{s} b_{s}^{\top}\right)}\right)-2\left(b_{s}^{1} \cdot b_{s}^{2}-b_{s, *}^{1} \cdot b_{s, *}^{2}\right) b_{s}^{1} \cdot b_{s}^{2} \sqrt{\tilde{a}_{s}^{1} \tilde{a}_{s}^{2}}}{4 \sqrt{\operatorname{det}\left(b_{s} b_{s}^{\top}\right)\left(\tilde{a}_{s}^{1}\left|b_{s}^{1}\right|^{2}+\tilde{a}_{s}^{2}\left|b_{s}^{2}\right|^{2}+2 \sqrt{\tilde{a}_{s}^{1} \tilde{a}_{s}^{2} \operatorname{det}\left(b_{s} b_{s}^{\top}\right)}\right)^{1 / 2}}}\right. \\
& \quad-\frac{\left(\tilde{a}_{s}^{1}\left|b_{s}^{1}\right|^{2}+\tilde{a}_{s}^{2}\left|b_{s}^{2}\right|^{2}+2 \sqrt{\tilde{a}_{s}^{1} \tilde{a}_{s}^{2} \operatorname{det}\left(b_{s} b_{s}^{\top}\right)}\right)^{1 / 2}}{2} \\
& \left.\quad+\frac{\left(\tilde{a}_{s}^{1}\left|b_{s, *}^{1}\right|^{2}+\tilde{a}_{s}^{2}\left|b_{s, *}^{2}\right|^{2}+2 \sqrt{\tilde{a}_{s}^{1} \tilde{a}_{s}^{2} \operatorname{det}\left(b_{s, *} b_{s, *}^{\top}\right)}\right)^{1 / 2}}{2}\right\} d s .
\end{aligned}
$$

Then by Theorem 3.2 in Jacod [18], it is sufficient to show that $\sum_{m=2}^{\left[\ell_{n} t / T\right]} E_{m}\left[\mathcal{X}_{m}^{n}\left(N_{s_{m}}-\right.\right.$ $\left.\left.N_{s_{m-1}}\right)\right] \rightarrow^{p} 0$ for any bounded $\mathcal{G}_{t}$-martingale $N=\left(N_{t}\right)_{0 \leq t \leq T}$ orthogonal to $W$.

Orthogonality of $N$ and $W$ yields

$$
E_{m}\left[\mathcal{X}_{m}^{n}\left(N_{s_{m}}-N_{s_{m-1}}\right)\right]=E_{m}\left[\dot{\mathcal{X}}_{m}^{n}\left(N_{s_{m}}-N_{s_{m-1}}\right)\right]
$$

where $\dot{\mathcal{X}}_{m}^{n}=-2^{-1} b_{n}^{-1 / 4} \bar{E}_{m}\left[\tilde{Z}_{2, m}^{\top} \partial_{\sigma} S_{m, *}^{-1} \tilde{Z}_{2, m}+2 \tilde{Z}_{1, m}^{\top} \partial_{\sigma} S_{m, *}^{-1} \tilde{Z}_{2, m}\right]$, and $\tilde{Z}_{1, m}$ and $\tilde{Z}_{2, m}$ are defined in (A.4).

Let $N^{\prime}=\left(N_{t}^{\prime}\right)_{0 \leq t \leq T}$ be another bounded $\mathcal{G}_{t}$-martingale. Then we obtain

$$
\begin{aligned}
\sum_{m=2}^{\left[\ell_{n} t / T\right]} E_{m}\left[\dot{\mathcal{X}}_{m}^{n}\left(N_{s_{m}}-N_{s_{m-1}}\right)\right]= & \sum_{m=2}^{\left[\ell_{n} t / T\right]} E_{m}\left[\dot{\mathcal{X}}_{m}^{n}\left(N_{s_{m}}^{\prime}-N_{s_{m-1}}^{\prime}\right)\right] \\
& +\sum_{m=2}^{\left[\ell_{n} t / T\right]} E_{m}\left[\dot{\mathcal{X}}_{m}^{n}\left(N_{s_{m}}-N_{s_{m}}^{\prime}-N_{s_{m-1}}+N_{s_{m-1}}^{\prime}\right)\right]
\end{aligned}
$$

and

$$
\begin{aligned}
& E\left[\left|\sum_{m=2}^{\left[\ell_{n} t / T\right]} E_{m}\left[\dot{\mathcal{X}}_{m}^{n}\left(N_{s_{m}}-N_{s_{m}}^{\prime}-N_{s_{m-1}}+N_{s_{m-1}}^{\prime}\right)\right]\right| 1_{\left\{\sum_{m} E_{m}\left[\left(\dot{\mathcal{X}}_{m}^{n}\right)^{2}\right] \leq K\right\}}\right] \\
& \leq E\left[\sum_{m=2}^{\ell_{n}} E_{m}\left[\left(\dot{\mathcal{X}}_{m}^{n}\right)^{2}\right]^{1 / 2} E_{m}\left[\left(N_{s_{m}}-N_{s_{m}}^{\prime}-N_{s_{m-1}}+N_{s_{m-1}}^{\prime}\right)^{2}\right]^{1 / 2} 1_{\left\{\sum_{m} E_{m}\left[\left(\dot{\mathcal{X}}_{m}^{n}\right)^{2}\right] \leq K\right\}}\right]
\end{aligned}
$$




$$
\begin{aligned}
& \leq E\left[\sum_{m=2}^{\ell_{n}} E_{m}\left[\left(\dot{\mathcal{X}}_{m}^{n}\right)^{2}\right] 1_{\left\{\sum_{m} E_{m}\left[\left(\dot{\mathcal{X}}_{m}^{n}\right)^{2}\right] \leq K\right\}}\right]^{1 / 2} E\left[\sum_{m=2}^{\ell_{n}}\left(N_{s_{m}}-N_{s_{m}}^{\prime}-N_{s_{m-1}}+N_{s_{m-1}}^{\prime}\right)^{2}\right]^{1 / 2} \\
& \leq \sqrt{K} E\left[\left(N_{T}-N_{T}^{\prime}\right)^{2}\right]^{1 / 2}
\end{aligned}
$$

for any positive constant $K$.

Since we have the tightness of $\sum_{m=2}^{\ell_{n}} E_{m}\left[\left(\dot{X}_{m}^{n}\right)^{2}\right]$ similarly to (7.2), we obtain the desired result if for any $\varepsilon>0$, we can find a bounded $\mathcal{G}_{t}$-martingale $N^{\prime}$ which satisfies $E\left[\left(N_{T}-N_{T}^{\prime}\right)^{2}\right] \leq$ $\varepsilon$ and $E_{m}\left[\dot{\mathcal{X}}_{m}^{n}\left(N_{s_{m}}^{\prime}-N_{s_{m-1}}^{\prime}\right)\right]=0$ for any $m$. We obtain such $N^{\prime}$ by setting $N_{t}^{\prime}=E\left[\tilde{N} \mid \mathcal{G}_{t}\right]$ for suitable $\tilde{N} \in \mathbf{N}$, where $\mathbf{N}$ is the set of finite sums of random variables $\mathbf{X} \prod_{j=1}^{l} g_{j}\left(\varepsilon_{i_{j}}^{n_{j}, k_{j}}\right)$ with bounded Borel functions $\left(g_{j}\right)_{j=1}^{l}$, an $\mathcal{F}^{(0)}$-measurable bounded random variable $\mathbf{X}, n_{1}, \ldots, n_{l} \in$ $\mathbb{N}, 1 \leq k_{1}, \ldots, k_{l} \leq 2$, and $i_{1}, \ldots, i_{l} \in \mathbb{Z}_{+}$. Then $\mathbf{N}$ is dense in $L^{2}\left(\Omega, \mathcal{G}_{T}, P\right)$ and we obtain $E_{m}\left[\dot{\dot{\mathcal{X}}_{m}^{n}}\left(N_{s_{m}}^{\prime}-N_{s_{m-1}}^{\prime}\right)\right]=0$ for any $\mathrm{m}$ if $n>\max _{j} n_{j}$.

Proof of Theorem 2.1. Since the parameter space $\Lambda$ is open, there exists $\varepsilon>0$ such that $O\left(\varepsilon, \sigma_{*}\right)=\left\{\sigma ;\left|\sigma-\sigma_{*}\right|<\varepsilon\right\} \subset \Lambda$. Then we have

$$
-\partial_{\sigma} H_{n}\left(\sigma_{*}, \hat{v}_{n}\right)=\int_{0}^{1} \partial_{\sigma}^{2} H_{n}\left(\sigma_{*}, \hat{v}_{n}\right)\left(\sigma_{*}+t\left(\hat{\sigma}_{n}-\sigma_{*}\right)\right)\left(\hat{\sigma}_{n}-\sigma_{*}\right) d t
$$

for $\hat{\sigma}_{n} \in O\left(\varepsilon, \sigma_{*}\right)$, by $\partial_{\sigma} H_{n}\left(\hat{\sigma}_{n}, \hat{v}_{n}\right)=0$.

Hence, we obtain $b_{n}^{1 / 4}\left(\hat{\sigma}_{n}-\sigma_{*}\right)=\tilde{\Gamma}_{1, n}^{-1} b_{n}^{-1 / 4} \partial_{\sigma} H_{n}\left(\sigma_{*}, \hat{v}_{n}\right)$ on $\left\{\operatorname{det} \tilde{\Gamma}_{1, n} \neq 0\right.$ and $\hat{\sigma}_{n} \in$ $\left.O\left(\varepsilon, \sigma_{*}\right)\right\}$, where $\tilde{\Gamma}_{1, n}=-b_{n}^{-1 / 2} \int_{0}^{1} \partial_{\sigma}^{2} H_{n}\left(\sigma_{*}+t\left(\hat{\sigma}_{n}-\sigma_{*}\right)\right) d t$. Then since Propositions 2.1, 6.1 and 7.1 yield $P\left[\operatorname{det} \tilde{\Gamma}_{1, n}=0\right] \rightarrow 0, P\left[\hat{\sigma}_{n} \notin O\left(\varepsilon, \sigma_{*}\right)\right] \rightarrow 0$ and $\tilde{\Gamma}_{1, n}^{-1} 1_{\left\{\operatorname{det} \tilde{\Gamma}_{1, n} \neq 0\right\}} \rightarrow^{p} \Gamma_{1}^{-1}$, we have $b_{n}^{1 / 4}\left(\hat{\sigma}_{n}-\sigma_{*}\right) \rightarrow^{s-\mathcal{L}} \Gamma_{1}^{-1 / 2} \mathcal{N}$ as $n \rightarrow \infty$ by Proposition 7.2.

Moreover, Propositions 2.1 and 7.1 ensure that $\hat{\Gamma}_{1, n} \rightarrow^{p} \Gamma_{1}$, which completes the proof.

\section{Proof of the LAN property}

To obtain the LAN property of our model, the arguments in the proof of Theorem 2.1 are essential. Indeed, by using Propositions 2.1 and 7.2, we obtain a LAMN-type property of the quasi-log-likelihood function $H_{n}$ with respect to $\sigma: H_{n}\left(\sigma_{*}+b_{n}^{-1 / 4} u_{1}, v_{*}\right)-H_{n}\left(\sigma_{*}, v_{*}\right)-$ $u_{1} \cdot b_{n}^{-1 / 4} \partial_{\sigma} H_{n}\left(\sigma_{*}, v_{*}\right)-u_{1}^{\top} b_{n}^{-1 / 2} \partial_{\sigma}^{2} H_{n}\left(\sigma_{*}, v_{*}\right) u_{1} / 2 \rightarrow^{p} 0$ as $n \rightarrow \infty$ for any $u_{1} \in \mathbb{R}^{d}$, and $\left(b_{n}^{-1 / 4} \partial_{\sigma} H_{n}\left(\sigma_{*}, v_{*}\right),-b_{n}^{-1 / 2} \partial_{\sigma}^{2} H_{n}\left(\sigma_{*}, v_{*}\right)\right) \rightarrow^{s-\mathcal{L}}\left(\Gamma_{1}^{1 / 2} \mathcal{N}, \Gamma_{1}\right)$, where $\mathcal{N}$ is a $d$-dimensional standard normal random variable independent of $\mathcal{F}$. On the other hand, under the assumptions of

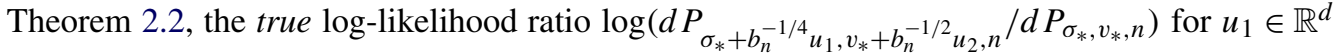
and $u_{2} \in \mathbb{R}^{2}$ is obtained as $-\left(Z_{1}^{\top} S_{1}^{-1} Z_{1}+\log \operatorname{det} S_{1}\right) / 2$ if we set $k_{n}=b_{n}$. We cannot apply the argument of Section 5 to this quantity because the estimate $\ell_{n} \rightarrow \infty$ is essential there. Therefore, we follow the approaches by Gloter and Jacod [12] to show the LAN property. We set a "subexperiment" and a "superexperiment", which are obtained by respectively removing and adding 
observations from the original experiment. The likelihood functions of these experiments have similar properties to $H_{n}$, and therefore we can prove the LAN properties for these experiments with the same limit distribution. We can prove that these results lead us to the LAN property of the original one.

Let $\mathcal{Z}=\left(\mathbb{R}^{8}\right)^{\mathbb{Z}_{+}}, \pi_{i}(z)=\left(x_{i}^{k, j}, t_{i}^{k}, e_{i}^{k}\right)_{j, k=1,2}$ for $i \in \mathbb{Z}_{+}$and $z=\left(x_{i^{\prime}}^{k, j}, t_{i^{\prime}}^{k}, e_{i^{\prime}}^{k}\right)_{i^{\prime} \in \mathbb{Z}_{+}, j, k=1,2} \in$ $\mathcal{Z}$. Let $\mathcal{H}=\mathfrak{B}\left(\left\{\pi_{i}^{-1}(A) ; i \in \mathbb{Z}_{+}, A \in \mathcal{B}\left(\mathbb{R}^{8}\right)\right\}\right), P_{\sigma_{*}^{\prime}, v_{*}^{\prime}}^{\prime}$ be the induced probability measure on $(\mathcal{Z}, \mathcal{H})$ by $\left(\left(Y_{S_{i}^{n, k}}^{j} 1_{\left\{i \leq \mathbf{J}_{k, n}\right\}}, S_{i}^{n, k} 1_{\left\{i \leq \mathbf{J}_{k, n}\right\}}, \varepsilon_{i}^{n, k} 1_{\left\{i \leq \mathbf{J}_{k, n}\right\}}\right)_{i \in \mathbb{Z}_{+}, j, k=1,2}\right)$ with a true value $\left(\sigma_{*}^{\prime}, v_{*}^{\prime}\right)$. We can ignore the event $\min _{j, m} k_{m}^{j} \leq 0$.

Let $\mathcal{H}^{\prime}=\mathfrak{B}\left(t_{i}^{k} ; i \in \mathbb{Z}_{+}, k=1,2\right), j_{0}^{k}=-1, j_{m}^{k}=\max \left\{i ; t_{i}^{k}<s_{m}\right\} \vee 0\left(1 \leq m \leq \ell_{n}\right), \mathbf{l}(0)=1$, $\mathbf{I}(m)=\min \left\{k ; t_{i}^{k}=\max _{i^{\prime}, k^{\prime}}\left\{t_{i^{\prime}}^{k^{\prime}}<s_{m}\right\}\right.$ for some $\left.i\right\}$ for $1 \leq m \leq \ell_{n}$,

$$
\begin{aligned}
& \mathcal{H}^{n, 0}=\mathfrak{B}\left(\left(x_{i+1}^{k, k}+e_{i+1}^{k}-x_{i}^{k, k}-e_{i}^{k}\right) 1_{\left\{i \notin\left\{j_{m}^{k}\right\}_{m}\right\}} ; i \in \mathbb{Z}_{+}, k=1,2\right) \vee \mathcal{H}^{\prime}, \\
& \mathcal{H}^{n, 1}=\mathfrak{B}\left(x_{0}^{k, k}, x_{i}^{k, k}+e_{i}^{k} ; i \in \mathbb{Z}_{+}, k=1,2\right) \vee \mathcal{H}^{\prime}, \\
& \mathcal{H}^{n, 2}=\mathcal{H}^{n, 1} \vee \mathfrak{B}\left(x_{j_{m}^{1(m)}}^{\mathbf{l}(m), j} ; 1 \leq m \leq \ell_{n}-1, j=1,2\right) \vee \mathfrak{B}\left(x_{j_{\ell_{n}}^{1}+1}^{1,1}, x_{j_{\ell_{n}}^{1}+1}^{1,2}\right) .
\end{aligned}
$$

Then we can see $\mathcal{H}^{n, 0} \subset \mathcal{H}^{n, 1} \subset \mathcal{H}^{n, 2}$ and

$$
\log \left(d P_{\sigma_{u}, v_{u}} / d P_{\sigma_{*}, v_{*}}\right)=\log \frac{d\left(\left.P_{\sigma_{u}, v_{u}}^{\prime}\right|_{\mathcal{H}^{n, 1}}\right)}{d\left(\left.P_{\sigma_{*}, v_{*}}^{\prime}\right|_{\mathcal{H}^{n, 1}}\right)} .
$$

Moreover, we obtain

$$
\begin{aligned}
\log & \frac{d\left(P_{\sigma_{u}, v_{u}}^{\prime} \mid \mathcal{H}^{n, l}\right)}{d\left(P_{\sigma_{*}, v_{*}}^{\prime} \mid \mathcal{H}^{n, l}\right)}\left(\left(Y_{S_{i}^{n, k}}^{j} 1_{\left\{i \leq \mathbf{J}_{k, n}\right\}}, S_{i}^{n, k} 1_{\left\{i \leq \mathbf{J}_{k, n}\right\}}, \varepsilon_{i}^{n, k} 1_{\left\{i \leq \mathbf{J}_{k, n}\right\}}\right)_{i \in \mathbb{Z}_{+}, j, k=1,2}\right) \\
= & H_{n}^{(l)}\left(\sigma_{u}, v_{u}\right)-H_{n}^{(l)}\left(\sigma_{*}, v_{*}\right)
\end{aligned}
$$

for $l=0$, where $\mu(t, \sigma)=\left(\mu^{1}(t, \sigma), \mu^{2}(t, \sigma)\right)^{\top}, Z_{m}^{(0)}(\sigma)=\left(\tilde{Y}^{k}\left(I_{i, m}^{k}\right)-\int_{I_{i, m}^{k}} \mu^{k}(t, \sigma) d t\right)_{i, k}$, and

$$
S_{m}^{(0)}(\sigma, v)=\left(\begin{array}{lc}
\operatorname{diag}\left(\left(\int_{I_{i, m}^{1}}\left|b_{t}^{1}\right|^{2} d t\right)_{i}\right) & \left(\int_{I_{i, m}^{1} \cap I_{j, m}^{2}} b_{t}^{1} \cdot b_{t}^{2} d t\right)_{i, j} \\
\left(\int_{I_{i, m}^{1} \cap I_{j, m}^{2}} b_{t}^{1} \cdot b_{t}^{2} d t\right)_{j, i} & \operatorname{diag}\left(\left(\int_{I_{j, m}^{2}}\left|b_{t}^{2}\right|^{2} d t\right)_{j}\right)
\end{array}\right)+\left(\begin{array}{cc}
v_{1} M_{1, m} & 0 \\
0 & v_{2} M_{2, m}
\end{array}\right)
$$

for $1 \leq m \leq \ell_{n}, H_{n}^{(0)}(\sigma, v)=-\sum_{m=1}^{\ell_{n}}\left\{\left(Z_{m}^{(0)}(\sigma)\right)^{\top}\left(S_{m}^{(0)}\right)^{-1}(\sigma, v) Z_{m}^{(0)}(\sigma)+\log \operatorname{det} S_{m}^{(0)}(\sigma, v)\right\} / 2$, $\sigma_{u}=\sigma_{*}+b_{n}^{-1 / 4} u_{1}$ and $v_{u}=v_{*}+b_{n}^{-1 / 2} u_{2}$ for $u=\left(u_{1}, u_{2}\right) \in \mathbb{R}^{d} \times \mathbb{R}^{2} . \mathcal{H}^{n, 0}$ and $\mathcal{H}^{n, 2}$ are $\sigma-$ fields for "subexperiment" and "superexperiment", respectively, while $\mathcal{H}^{n, 1}$ is the one for the original one. 
To obtain similar formula to (8.2) for $l=2$, let $\mathbf{R}_{m}=S_{K_{m}^{1}}^{n, 1} \vee S_{K_{m}^{2}}^{n, 2}, \mathbf{Q}_{m}^{k}=S_{K_{m}^{k}}^{n, k}, \mathbf{Q}_{m, \pm}^{k}=S_{K_{m}^{k} \pm 1}^{n, k}$,

$$
\begin{aligned}
& \tilde{\mathbf{Y}}_{m,-}^{k}(\sigma)=\tilde{Y}_{K_{m-1}^{k}+1}^{k}-Y_{\mathbf{R}_{m-1}}^{k}-\int_{\mathbf{R}_{m-1}}^{\mathbf{Q}_{m-1,+}^{k}} \mu^{k}(t, \sigma) d t, \\
& \tilde{\mathbf{Y}}_{m,+}^{k}(\sigma)=\left\{\begin{array}{ll}
Y_{\mathbf{Q}_{m}^{k}}^{k}-\tilde{Y}_{K_{m}^{k}-1}^{k}-\int_{\mathbf{Q}_{m,-}^{k}}^{\mathbf{Q}_{m}^{k}} \mu^{k}(t, \sigma) d t, & \text { if } \mathbf{Q}_{m}^{k}=\mathbf{R}_{m}, \\
\left(\tilde{Y}^{k}\left(I_{k_{m}^{k}, m}^{k}\right)-\int_{I_{k}^{k}, m}^{k} \mu^{k}(t, \sigma) d t\right. \\
Y_{\mathbf{R}_{m}}^{k}-\tilde{Y}_{K_{m}^{k}}^{k}-\int_{\mathbf{Q}_{m}^{k}}^{\mathbf{R}_{m}} \mu^{k}(t, \sigma) d t
\end{array}\right),
\end{aligned}
$$

$\mathbf{Y}_{m, 0}=\varepsilon_{K_{m}^{k}}^{n, k}$ if $\mathbf{Q}_{m}^{3-k}<\mathbf{R}_{m}, \mathbf{Y}_{m, 0}=\left(\varepsilon_{K_{m}^{1}}^{n, 1}, \varepsilon_{K_{m}^{2}}^{n, 2}\right)^{\top}$ if $\mathbf{Q}_{m}^{1}=\mathbf{Q}_{m}^{2}$, and

$$
Z_{m}^{(2)}(\sigma)=\left(\left(\left(\tilde{\mathbf{Y}}_{m,-}^{k}(\sigma)\right)^{\top},\left(\tilde{Y}^{k}\left(I_{i, m}^{k}\right)-\int_{I_{i, m}^{k}} \mu^{k}(t, \sigma) d t\right)_{1 \leq i<k_{m}^{k}}^{\top},\left(\tilde{\mathbf{Y}}_{m,+}^{k}(\sigma)\right)^{\top}\right)_{k=1}^{2}, \mathbf{Y}_{m, 0}^{\top}\right)^{\top}
$$

for $2 \leq m \leq \ell_{n}-1$. Then (8.2) holds for $l=2$, where $\bar{I}_{1, m}^{k}=\left[\mathbf{R}_{m-1}^{k}, \mathbf{Q}_{m-1,+}^{k}\right), \bar{I}_{i, m}^{k}=I_{i-1, m}^{k}$ for $2 \leq i \leq k_{m}^{k}+1$,

$$
\begin{aligned}
& \mathbf{E}(v)=v_{3-k}, \quad k_{m}^{(2), k}=k_{m}^{k}+2, \quad k_{m}^{(2), 3-k}=k_{m}^{3-k}+1, \quad \text { and } \\
& \bar{I}_{k_{m}^{(2), k}, m}^{k}=\left[\mathbf{Q}_{m}^{k}, \mathbf{R}_{m}\right), \quad \text { if } \mathbf{Q}_{m}^{k}<\mathbf{R}_{m}, \\
& \mathbf{E}(v)=\operatorname{diag}\left(v_{1}, v_{2}\right) \quad \text { and } \quad\left(k_{m}^{(2), 1}, k_{m}^{(2), 2}\right)=\left(k_{m}^{1}+1, k_{m}^{2}+1\right) \quad \text { if } \mathbf{Q}_{m}^{1}=\mathbf{Q}_{m}^{2} \text {, } \\
& \left(M_{j, m}^{(2)}\right)_{i i^{\prime}}=2 \delta_{i i^{\prime}}-1_{\left\{\left|i-i^{\prime}\right|=1\right\}}-1_{\left\{i=i^{\prime}=1\right\}}-1_{\left\{i=i^{\prime}=k_{m}^{(2), j}\right\}}, \\
& S_{m}^{(2)}(\sigma, v)
\end{aligned}
$$

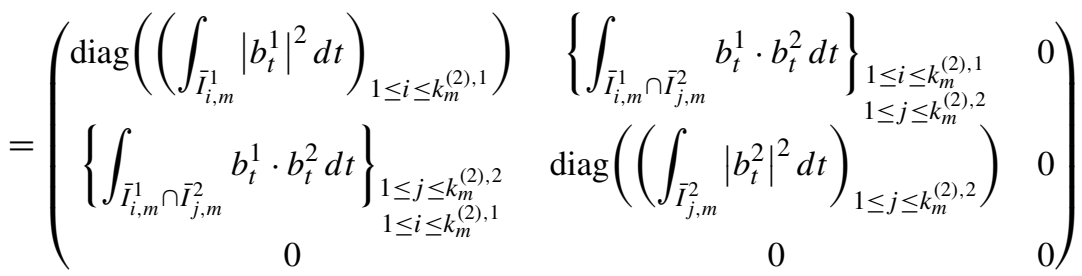

$$
\begin{aligned}
& +\left(\begin{array}{ccc}
v_{1} M_{1, m}^{(2)} & 0 & 0 \\
0 & v_{2} M_{2, m}^{(2)} & 0 \\
0 & 0 & \mathbf{E}(v)
\end{array}\right)
\end{aligned}
$$


for $2 \leq m \leq \ell_{n}-1, Z_{m}^{(2)}, M_{m}^{(2)}$, and $S_{m}^{(2)}$ are similarly defined for $m=1, \ell_{n}$, and

$$
H_{n}^{(2)}(\sigma, v)=-\frac{1}{2} \sum_{m=1}^{\ell_{n}}\left\{\left(Z_{m}^{(2)}(\sigma)\right)^{\top} S_{m}^{(2)}(\sigma, v)^{-1} Z_{m}^{(2)}(\sigma)+\log \operatorname{det} S_{m}^{(2)}(\sigma, v)\right\}
$$

The log-likelihood functions $H_{n}^{(0)}$ and $H_{n}^{(2)}$ of "subexperiment" and "superexperiment", respectively, have similar properties to that of $H_{n}$, and hence we can prove convergence of likelihood ratios. Gloter and Jacod [12] showed that convergence of likelihood ratios of "subexperiment" and "superexperiment" imply convergence of that of the original experiment (Theorem 4.1). Here, we use a slight extension of their result. The proof is straightforward. Let $\mathbf{U}_{\sigma, v}^{n, l}=d\left(\left.P_{\sigma, v}^{\prime}\right|_{\mathcal{H}^{n, l}}\right) / d\left(\left.P_{\sigma_{*}, v_{*}}^{\prime}\right|_{\mathcal{H}^{n, l}}\right), K \in \mathbb{N}$, and $\left\{\sigma_{n}^{k}\right\}_{n \in \mathbb{N}, 1 \leq k \leq K} \subset \Lambda$ and $\left.v_{n}^{k}\right\}_{n \in \mathbb{N}, 1 \leq k \leq K} \subset$ $(0, \infty) \times(0, \infty)$ be arbitrary sequences.

Theorem 8.1. Suppose that $\left(\mathbf{U}_{\sigma_{n}^{1}, v_{n}^{1}}^{n, l}, \ldots, \mathbf{U}_{\sigma_{n}^{K}, v_{n}^{K}}^{n, l}\right)$ converges in law under $P_{\sigma_{*}, v_{*}}^{\prime n}$ to a limit $Y=$ $\left(Y^{1}, \ldots, Y^{K}\right)$ with $0<Y^{k}<\infty$ almost surely and $E\left[Y^{k}\right]=1$ for $l=0,2$ and $1 \leq k \leq K$. Then the same convergence holds for $l=1$.

We will show the LAN properties of "subexperiment" and 'superexperiment'. Then Theorem 8.1 leads to the LAN property of the original one.

First, Taylor's formula yields

$$
\begin{aligned}
& H_{n}^{(l)}\left(\sigma_{u}, v_{u}\right)-H_{n}^{(l)}\left(\sigma_{*}, v_{*}\right) \\
& =b_{n}^{-1 / 4} \partial_{\sigma} H_{n}^{(l)}\left(\sigma_{*}, v_{*}\right) \cdot u_{1}+2^{-1} b_{n}^{-1 / 2} u_{1}^{\top} \partial_{\sigma}^{2} H_{n}^{(l)}\left(\sigma_{*}, v_{*}\right) u_{1}+b_{n}^{-1 / 2} \partial_{v} H_{n}^{(l)}\left(\sigma_{*}, v_{*}\right) \cdot u_{2} \\
& \quad+2^{-1} b_{n}^{-1} u_{2}^{\top} \partial_{v}^{2} H_{n}^{(l)}\left(\sigma_{*}, v_{*}\right) u_{2}+\int_{0}^{1} \int_{0}^{1} \sum_{i, j} b_{n}^{-\frac{3}{4}} \partial_{v_{i}} \partial_{\sigma_{j}} H_{n}^{(l)}\left(\sigma_{t u}, v_{s u}\right) u_{2, i} u_{1, j} d s d t \\
& \quad+\int_{0}^{1} \frac{(1-t)^{2}}{2} \sum_{i, j, k} b_{n}^{-\frac{3}{4}} \partial_{\sigma_{i}} \partial_{\sigma_{j}} \partial_{\sigma_{k}} H_{n}^{(l)}\left(\sigma_{t u}, v_{*}\right) u_{1, i} u_{1, j} u_{1, k} d t \\
& \quad+\int_{0}^{1} \frac{(1-t)^{2}}{2} \sum_{i, j, k} b_{n}^{-\frac{3}{2}} \partial_{v_{i}} \partial_{v_{j}} \partial_{v_{k}} H_{n}^{(l)}\left(\sigma_{*}, v_{t u}\right) u_{2, i} u_{2, j} u_{2, k} d t
\end{aligned}
$$

We examine the limit of each term on the right-hand side. First, we prepare an auxiliary lemma. Let $\mathfrak{P}_{q}=\left\{\alpha=\left(\alpha_{1}, \ldots, \alpha_{L}\right) ; L \in \mathbb{N}, 1 \leq \alpha_{l} \leq q, \sum_{l=1}^{L} \alpha_{l}=q\right\}, M_{m, *}^{(0)}=M_{m, *}$, $M_{m, *}^{(2)}=\operatorname{diag}\left(v_{1, *} M_{1, m}^{(2)}, v_{2, *} M_{2, m}^{(2)}, \mathbf{E}\left(v_{*}\right)\right)$,

$$
\mathbf{Y}_{2, m,+}^{k}= \begin{cases}-\varepsilon_{K_{m}^{k}-1}^{n, k}, & \text { if } \mathbf{Q}_{m}^{k} \geq \mathbf{Q}_{m}^{3-k}, \\ \left(\varepsilon_{\left.K_{m}^{n, k}-\varepsilon_{K_{m}^{k}-1}^{n, k},-\varepsilon_{K_{m}^{k}}^{n, k}\right),}\right. & \text { if } \mathbf{Q}_{m}^{k}<\mathbf{Q}_{m}^{3-k}\end{cases}
$$


$Z_{2, m}^{(0)}=\left(\varepsilon_{K_{m-1}^{k}+i+1}^{n, k}-\varepsilon_{K_{m-1}^{k, k}+i}^{n, k}\right)_{1 \leq i \leq k_{m}^{k}, k=1,2}$, and

$$
Z_{2, m}^{(2)}=\left(\left(\varepsilon_{K_{m-1}^{k}+1}^{n, k},\left(\varepsilon_{K_{m-1}^{k}+i+1}^{n, k}-\varepsilon_{K_{m-1}^{k}+i}^{n, k}\right)_{1 \leq i<k_{m}^{k}}^{\top},\left(\mathbf{Y}_{2, m,+}^{k}\right)^{\top}\right)_{k=1,2}, \mathbf{Y}_{m, 0}^{\top}\right)^{\top}
$$

for $2 \leq m \leq \ell_{n}-1$. Though we can similarly define $Z_{2, m}^{(0)}$ and $Z_{2, m}^{(2)}$ for $m=1, \ell_{n}$ so that they satisfy the following lemma, we omit it to avoid redundancy. We also omit discussions for $m=$ $1, \ell_{n}$ throughout the rest of this section.

Lemma 8.1. Assume (A1" ). Let $p \in \mathbb{N}, l \in\{0,2\}, 1 \leq m \leq \ell_{n}$ and let $\dot{\mathbf{S}}_{m}$ be a $\mathcal{G}_{s_{m-1}}$-measurable random matrix of suitable size. Then there exists a positive constant $C_{p}$ depending only on $p$ such that
(i) $\left|E_{m}\left[\left(\left(Z_{2, m}^{(l)}\right)^{\top} \dot{\mathbf{S}}_{m} Z_{2, m}^{(l)}\right)^{p}\right]\right| \leq C_{p} \sum_{\alpha=\left(\alpha_{1}, \ldots, \alpha_{L}\right) \in \mathfrak{P}_{p}} \prod_{l=1}^{L}\left|\operatorname{tr}\left(\left(\dot{\mathbf{S}}_{m} M_{m, *}^{(l)}\right)^{\alpha_{l}}\right)\right|$.
(ii) $E_{m}\left[\bar{E}_{m}\left[\left(Z_{2, m}^{(l)}\right)^{\top} \dot{\mathbf{S}}_{m} Z_{2, m}^{(l)}\right]^{4}\right]=12 \operatorname{tr}\left(\left(M_{m, *}^{(l)} \dot{\mathbf{S}}_{m}\right)^{2}\right)^{2}+48 \operatorname{tr}\left(\left(M_{m, *}^{(l)} \dot{\mathbf{S}}_{m}\right)^{4}\right)$.

Proof. (i) Let $U_{m}^{(l)}$ be an orthogonal matrix and $\Lambda_{m}^{(l)}$ be a diagonal matrix satisfying $U_{m}^{(l)} M_{m, *}^{(l)}\left(U_{m}^{(l)}\right)^{\top}=\Lambda_{m}^{(l)}$. Then since $Z_{2, m}^{(l)} \sim N\left(0, M_{m, *}^{(l)}\right)$, a similar estimate to (A.5) yields

$$
E_{m}\left[\left(\left(Z_{2, m}^{(l)}\right)^{\top} \dot{\mathbf{S}}_{m} Z_{2, m}^{(l)}\right)^{p}\right]=\sum_{i_{1}, \ldots, i_{2 p}} \prod_{q=1}^{p}\left(U_{m}^{(l)} \dot{\mathbf{S}}_{m}\left(U_{m}^{(l)}\right)^{\top}\right)_{i_{2 q-1} i_{2 q}} \sum_{\left(l_{2 q^{\prime}-1}, l_{2 q^{\prime}}\right)_{q^{\prime}=1}^{p}} \prod_{q^{\prime}=1}^{p}\left(\Lambda_{m}^{(l)}\right)_{l_{2 q^{\prime}-1}, l_{2 q^{\prime}}},
$$

where the second summation on the right-hand side is over all disjoint pairs $\left(l_{2 q^{\prime}-1}, l_{2 q^{\prime}}\right)_{q^{\prime}=1}^{p}$ of variables $i_{1}, \ldots, i_{2 p}$.

(ii)

$$
\begin{aligned}
E_{m}[ & \left.\left(\operatorname{tr}\left(M_{m, *}^{(l)} \dot{\mathbf{S}}_{m}\right)-\left(Z_{2, m}^{(l)}\right)^{\top} \dot{\mathbf{S}}_{m} Z_{2, m}^{(l)}\right)^{4}\right] \\
= & \sum_{r=0}^{4} \frac{(-1)^{r} 4 !}{r !(4-r) !}\left(\operatorname{tr}\left(M_{m, *}^{(l)} \dot{\mathbf{S}}_{m}\right)\right)^{r} E_{m}\left[\left(\left(Z_{2, m}^{(l)}\right)^{\top} \dot{\mathbf{S}}_{m} Z_{2, m}^{(l)}\right)^{4-r}\right] \\
= & \operatorname{tr}\left(M_{m, *}^{(l)} \dot{\mathbf{S}}_{m}\right)^{4}-4 \operatorname{tr}\left(M_{m, *}^{(l)} \dot{\mathbf{S}}_{m}\right)^{4}+6 \operatorname{tr}\left(M_{m, *}^{(l)} \dot{\mathbf{S}}_{m}\right)^{2}\left(\operatorname{tr}\left(M_{m, *}^{(l)} \dot{\mathbf{S}}_{m}\right)^{2}+2 \operatorname{tr}\left(\left(M_{m, *}^{(l)} \dot{\mathbf{S}}_{m}\right)^{2}\right)\right) \\
& \quad-4 \operatorname{tr}\left(M_{m, *}^{(l)} \dot{\mathbf{S}}_{m}\right)\left(\operatorname{tr}\left(M_{m, *}^{(l)} \dot{\mathbf{S}}_{m}\right)^{3}+6 \operatorname{tr}\left(M_{m, *}^{(l)} \dot{\mathbf{S}}_{m}\right) \operatorname{tr}\left(\left(M_{m, *}^{(l)} \dot{\mathbf{S}}_{m}\right)^{2}\right)+8 \operatorname{tr}\left(\left(M_{m, *}^{(l)} \dot{\mathbf{S}}_{m}\right)^{3}\right)\right) \\
& +\left\{\operatorname{tr}\left(M_{m, *}^{(l)} \dot{\mathbf{S}}_{m}\right)^{4}+12 \operatorname{tr}\left(M_{m, *}^{(l)} \dot{\mathbf{S}}_{m}\right)^{2} \operatorname{tr}\left(\left(M_{m, *}^{(l)} \dot{\mathbf{S}}_{m}\right)^{2}\right)+12 \operatorname{tr}\left(\left(M_{m, *}^{(l)} \dot{\mathbf{S}}_{m}\right)^{2}\right)^{2}\right. \\
& \left.+32 \operatorname{tr}\left(M_{m, *}^{(l)} \dot{\mathbf{S}}_{m}\right) \operatorname{tr}\left(\left(M_{m, *}^{(l)} \dot{\mathbf{S}}_{m}\right)^{3}\right)+48 \operatorname{tr}\left(\left(M_{m, *}^{(l)} \dot{\mathbf{S}}_{m}\right)^{4}\right)\right\} \\
= & 12 \operatorname{tr}\left(\left(M_{m, *}^{(l)} \dot{\mathbf{S}}_{m}\right)^{2}\right)^{2}+48 \operatorname{tr}\left(\left(M_{m, *}^{(l)} \dot{\mathbf{S}}_{m}\right)^{4}\right) .
\end{aligned}
$$

Lemma 8.2. Assume (A1") and (A2). Then

1. $\sup _{\sigma}\left|b_{n}^{-1 / 2} \partial_{\sigma}^{k}\left(H_{n}^{(l)}\left(\sigma, v_{*}\right)-H_{n}^{(l)}\left(\sigma_{*}, v_{*}\right)\right)-\partial_{\sigma}^{k} \mathcal{Y}_{1}(\sigma)\right| \rightarrow^{p} 0$, 
2. $\sup _{v}\left|b_{n}^{-1} \partial_{v}^{k}\left(H_{n}^{(l)}\left(\sigma_{*}, v\right)-H_{n}^{(l)}\left(\sigma_{*}, v_{*}\right)\right)-\partial_{v}^{k} \mathcal{Y}_{2}(v)\right| \rightarrow^{p} 0$,

3. $\sup _{\sigma, v}\left|b_{n}^{-3 / 4} \partial_{\sigma} \partial_{v} H_{n}^{(l)}(\sigma, v)\right| \rightarrow^{p} 0$

as $n \rightarrow \infty$ for $0 \leq k \leq 3$ and $l=0,2$.

Proof. 1. For any $\varepsilon>0,\left(\varepsilon \mathcal{E}+M_{j, m}^{(2)}\right)^{-1}$ has a similar decomposition to (4.4) by replacing $p_{1}, \ldots, p_{k_{m}^{j}-1}, p_{k_{m}^{j}}$ by $p_{1}^{\prime}, \ldots, p_{k_{m}^{j}-1}^{\prime}, p_{k_{m}^{j}}^{\prime}-1$. Then, We obtain the desired results by a similar argument to the proof of Proposition 2.1 together with Lemmas 4.1 and 8.1, the results in Section 8 of [12], and similar estimates to Lemmas 5.1, 4.2 and 4.3. Estimate for the quantity corresponding to $\Lambda_{1}$ in Lemma 5.2 is obtained since $\left\{\left(\left(\varepsilon \mathcal{E}+M_{j, m}^{(2)}\right)^{-1}\right)_{k k}\right\}_{k=1}^{\left[k_{m}^{j} / 2\right]}$ is nonincreasing similarly to Lemma 4.2 , and

$$
\left(\left(c_{j} v_{j, *}^{-1} \mathcal{E}+M_{j, m}^{(2)}\right)^{-1}\right)_{11}=\frac{\prod_{l=1}^{k_{m}^{j}-1} p_{l}^{\prime}\left(c_{j} v_{j, *}^{-1}\right)}{\left(p_{k_{m}^{j}}^{\prime}\left(c_{j} v_{j, *}^{-1}\right)-1\right) \prod_{l=1}^{k_{m}^{j}-1} p_{l}^{\prime}\left(c_{j} v_{j, *}^{-1}\right)}=O_{p}\left(b_{n}^{1 / 2}\right) .
$$

2. Similarly to (5.1), we obtain

$$
b_{n}^{-1} \partial_{v}^{l} H_{n}^{(l)}(\sigma, v)=-\frac{1}{2} b_{n}^{-1} \sum_{m}\left\{\operatorname{tr}\left(\partial_{v}^{l}\left(S_{m}^{(l)}\right)^{-1} S_{m, *}^{(l)}\right)+\partial_{v}^{l} \log \operatorname{det} S_{m}^{(l)}\right\}+o_{p}(1) .
$$

Let $k_{m}^{(0), j}=k_{m}^{j}$ for $j=1,2, \tilde{D}_{1, m}^{(l)}=\left(\left(S_{m}^{(l)}\right)_{i, i^{\prime}}\right)_{1 \leq i, i^{\prime} \leq k_{m}^{(l), 1}}$,

$$
\begin{aligned}
& \tilde{D}_{2, m}^{(0)}=\left(\left(S_{m}^{(0)}\right)_{j, j^{\prime}}\right)_{k_{m}^{(0), 1}<j, j^{\prime} \leq k_{m}^{(0), 1}+k_{m}^{(0), 2},} \\
& \tilde{D}_{2, m}^{(2)}=\operatorname{diag}\left(\left(\left(S_{m}^{(2)}\right)_{j, j^{\prime}}\right)_{k_{m}^{(2), 1}<j, j^{\prime} \leq k_{m}^{(2), 1}+k_{m}^{(2), 2}}, \mathbf{E}(v)\right), \\
& \hat{G}_{m}^{(0)}=\left(\tilde{D}_{1, m}^{(0)}\right)^{-1 / 2}\left\{\int_{I_{i, m}^{1} \cap I_{j, m}^{2}} b_{t}^{1} \cdot b_{t}^{2} d t\right\}_{1 \leq i \leq k_{m}^{(0), 1}, 1 \leq j \leq k_{m}^{(0), 2}}\left(\tilde{D}_{2, m}^{(0)}\right)^{-1 / 2}, \\
& \hat{G}_{m}^{(2)}=\left(\tilde{D}_{1, m}^{(2)}\right)^{-1 / 2}\left\{\int_{\bar{I}_{i, m}^{1} \cap \bar{I}_{j, m}^{2}} b_{t}^{1} \cdot b_{t}^{2} d t 1_{\left\{j \leq k_{m}^{(2), 2}\right\}}\right\}_{1 \leq i \leq k_{m}^{(2), 1}, 1 \leq j \leq \tilde{k}_{m}^{(2), 2}}\left(\tilde{D}_{2, m}^{(2)}\right)^{-1 / 2},
\end{aligned}
$$

and $\tilde{D}_{m}^{(l)}=\operatorname{diag}\left(\tilde{D}_{1, m}^{(l)}, \tilde{D}_{2, m}^{(l)}\right)$ for $l=0,2$, where $\tilde{k}_{m}^{(2), 2}$ is the size of $\tilde{D}_{2, m}^{(2)}$. Then we obtain

$$
\begin{aligned}
& \operatorname{tr}\left(\left(S_{m}^{(l)}\right)^{-1} S_{m, *}^{(l)}\right) \\
& \quad=\operatorname{tr}\left(\left(\tilde{D}_{m}^{(l)}\right)^{-1 / 2}\left(\begin{array}{cc}
\mathcal{E} & \hat{G}_{m}^{(l)} \\
\left(\hat{G}_{m}^{(l)}\right)^{\top} & \mathcal{E}
\end{array}\right)^{-1}\left(\tilde{D}_{m}^{(l)}\right)^{-1 / 2}\left(\tilde{D}_{m, *}^{(l)}\right)^{1 / 2}\left(\begin{array}{cc}
\mathcal{E} & \hat{G}_{m, *}^{(l)} \\
\left(\hat{G}_{m, *}^{(l)}\right)^{\top} & \mathcal{E}
\end{array}\right)\left(\tilde{D}_{m, *}^{(l)}\right)^{1 / 2}\right) \\
& \quad=\sum_{p=0}^{\infty}\left\{\operatorname { t r } \left(\left(\tilde{D}_{1, m}^{(l)}\right)^{-1 / 2}\left(\hat{G}_{m}^{(l)}\left(\hat{G}_{m}^{(l)}\right)^{\top}\right)^{p}\left(\tilde{D}_{1, m}^{(l)}\right)^{-1 / 2} \tilde{D}_{1, m, *}^{(l)}\right.\right.
\end{aligned}
$$




$$
\begin{aligned}
& \left.-\left(\tilde{D}_{1, m}^{(l)}\right)^{-1 / 2}\left(\hat{G}_{m}^{(l)}\left(\hat{G}_{m}^{(l)}\right)^{\top}\right)^{p} \hat{G}_{m}^{(l)}\left(\tilde{D}_{2, m}^{(l)}\right)^{-1 / 2}\left(\tilde{D}_{2, m, *}^{(l)}\right)^{1 / 2}\left(\hat{G}_{m, *}^{(l)}\right)^{\top}\left(\tilde{D}_{1, m, *}^{(l)}\right)^{1 / 2}\right) \\
& +\operatorname{tr}\left(\left(\tilde{D}_{2, m}^{(l)}\right)^{-1 / 2}\left(\left(\hat{G}_{m}^{(l)}\right)^{\top} \hat{G}_{m}^{(l)}\right)^{p}\left(\tilde{D}_{2, m}^{(l)}\right)^{-1 / 2} \tilde{D}_{2, m, *}^{(l)}\right. \\
& \left.\left.-\left(\tilde{D}_{2, m}^{(l)}\right)^{-1 / 2}\left(\hat{G}_{m}^{(l)}\right)^{\top}\left(\hat{G}_{m}^{(l)}\left(\hat{G}_{m}^{(l)}\right)^{\top}\right)^{p}\left(\tilde{D}_{1, m}^{(l)}\right)^{-1 / 2}\left(\left(\tilde{D}_{1, m, *}^{(l)}\right)\right)^{1 / 2} \hat{G}_{m, *}^{(l)}\left(\tilde{D}_{2, m, *}^{(l)}\right)^{1 / 2}\right)\right\} .
\end{aligned}
$$

Since $\left\|\left(\tilde{D}_{j, m}^{(l)}\right)^{-1 / 2} \tilde{D}_{j, m, *}^{(l)}\left(\tilde{D}_{j, m}^{(l)}\right)^{-1 / 2}\right\|=O_{p}(1)$, terms involving $\hat{G}_{m}^{(l)}$ on the right-hand side are $O_{p}\left(b_{n}^{1 / 2} \ell_{n}^{-1}\right)$. Therefore, we have

$$
\begin{aligned}
& b_{n}^{-1} \operatorname{tr}\left(\left(S_{m}^{(l)}\right)^{-1} S_{m, *}^{(l)}\right) \\
& =b_{n}^{-1} \sum_{j=1}^{2} \operatorname{tr}\left(\left(\tilde{D}_{j, m}^{(l)}\right)^{-1} \tilde{D}_{j, m, *}^{(l)}\right)+o_{p}\left(\ell_{n}^{-1}\right) \\
& =b_{n}^{-1} \sum_{j=1}^{2} \frac{v_{j, *}}{v_{j}} \operatorname{tr}\left(\mathcal{E}_{k_{m}^{j}}-\left(\tilde{D}_{j, m}^{(l)}\right)^{-1}\left(\tilde{D}_{j, m}^{(l)}-v_{j} v_{j, *}^{-1} \tilde{D}_{j, m, *}^{(l)}\right)\right)+o_{p}\left(\ell_{n}^{-1}\right) \\
& =T \ell_{n}^{-1} \sum_{j=1}^{2} \hat{a}_{m}^{j} \frac{v_{j, *}}{v_{j}}+o_{p}\left(\ell_{n}^{-1}\right) .
\end{aligned}
$$

Similarly, we have $b_{n}^{-1} \operatorname{tr}\left(\partial_{v}^{k}\left(S_{m}^{(l)}\right)^{-1} S_{m, *}^{(l)}\right)=T \ell_{n}^{-1} \sum_{j=1}^{2} \hat{a}_{m}^{j} \partial_{v}^{k} \frac{v_{j, *}}{v_{j}}+o_{p}\left(\ell_{n}^{-1}\right)$. Moreover, we obtain

$$
\begin{aligned}
b_{n}^{-1} \partial_{v}^{k} \log \frac{\operatorname{det} S_{m}^{(l)}}{\operatorname{det} S_{m, *}^{(l)}} & \\
= & \sum_{j=1}^{2} b_{n}^{-1} \partial_{v}^{k} \log \operatorname{det}\left(\left(\tilde{D}_{j, m, *}^{(l)}\right)^{-1} \tilde{D}_{j, m}^{(l)}\right)+b_{n}^{-1} \partial_{v}^{k} \log \operatorname{det}\left(\mathcal{E}-\hat{G}_{m}^{(l)}\left(\hat{G}_{m}^{(l)}\right)^{\top}\right) \\
& \quad-b_{n}^{-1} \partial_{v}^{k} \log \operatorname{det}\left(\mathcal{E}-\hat{G}_{m, *}^{(l)}\left(\hat{G}_{m, *}^{(l)}\right)^{\top}\right) \\
= & b_{n}^{-1} \sum_{j=1}^{2} \partial_{v}^{k} \log \operatorname{det}\left(v_{j} v_{j, *}^{-1} \mathcal{E}_{k_{m}^{j}}+\left(\tilde{D}_{j, m, *}^{(l)}\right)^{-1}\left(\tilde{D}_{j, m}^{(l)}-v_{j} v_{j, *}^{-1} \tilde{D}_{j, m, *}^{(l)}\right)\right)+o_{p}\left(\ell_{n}^{-1}\right) \\
= & T \ell_{n}^{-1} \sum_{j=1}^{2} \hat{a}_{m}^{j} \partial_{v}^{k} \log \left(v_{j} v_{j, *}^{-1}\right)+o_{p}\left(\ell_{n}^{-1}\right) .
\end{aligned}
$$

3. Since $\partial_{v} \log \operatorname{det} S_{m}^{(l)}=\operatorname{tr}\left(\partial_{v} S_{m}^{(l)}\left(S_{m}^{(l)}\right)^{-1}\right)$, we have $b_{n}^{-3 / 4} \partial_{\sigma} \partial_{v} H_{n}^{(l)}(\sigma, v)=-\frac{1}{2} b_{n}^{-3 / 4} \sum_{m}\left\{\operatorname{tr}\left(\partial_{\sigma} \partial_{v}\left(S_{m}^{(l)}\right)^{-1} S_{m, *}^{(l)}\right)+\partial_{\sigma} \partial_{v} \log \operatorname{det} S_{m}^{(l)}\right\}+o_{p}(1)=o_{p}(1)$. 
Sobolev's inequality and similar estimates for $\partial_{\sigma} \partial_{v}^{2}$ and $\partial_{\sigma}^{2} \partial_{v}$ yield the results.

The following lemma completes the proof of the LAN properties of "subexperiment" and "superexperiment".

Lemma 8.3. Assume (A1") and (A2). Then $\left(b_{n}^{-1 / 4} \partial_{\sigma} H_{n}^{(l)}\left(\sigma_{*}, v_{*}\right)^{\top}, b_{n}^{-1 / 2} \times\right.$ $\left.\partial_{v} H_{n}^{(l)}\left(\sigma_{*}, v_{*}\right)^{\top}\right)^{\top} \rightarrow^{s-\mathcal{L}} \operatorname{diag}\left(\Gamma_{1}^{1 / 2}, \Gamma_{2}^{1 / 2}\right) \tilde{\mathcal{N}}$ for $l=0,2$, where $\tilde{\mathcal{N}}$ is a $(d+2)$-dimensional normal random variable independent of $\mathcal{F}$.

Proof. Let $Z_{m, *}^{(l)}=Z_{m}^{(l)}\left(\sigma_{*}\right), \tilde{\mathcal{X}}_{m}^{n, 1}=-b_{n}^{-1 / 4} \bar{E}_{m}\left[\left(Z_{m, *}^{(l)}\right)^{\top} \partial_{\sigma}\left(S_{m, *}^{(l)}\right)^{-1} Z_{m, *}^{(l)}\right] / 2, \tilde{\mathcal{X}}_{m}^{n, 2}=-b_{n}^{-1 / 2} \times$ $\bar{E}_{m}\left[\left(Z_{m, *}^{(l)}\right)^{\top} \partial_{v}\left(S_{m, *}^{(l)}\right)^{-1} Z_{m, *}^{(l)}\right] / 2$, and $\tilde{\mathcal{X}}_{m}^{n}=\left(\left(\tilde{\mathcal{X}}_{m}^{n, 1}\right)^{\top},\left(\tilde{\mathcal{X}}_{m}^{n, 2}\right)^{\top}\right)^{\top}$, then we have

$$
\begin{aligned}
\sum_{m=1}^{\left[\ell_{n} t / T\right]} E_{m}\left[\left|\tilde{\mathcal{X}}_{m}^{n}\right|^{2} 1_{\left\{\left|\tilde{\mathcal{X}}_{m}^{n}\right|>\varepsilon\right\}}\right] \leq & \frac{C}{\varepsilon^{2}} b_{n}^{-1} \sum_{m} E_{m}\left[\left|\left(Z_{m, *}^{(l)}\right)^{\top} \partial_{\sigma}\left(S_{m, *}^{(l)}\right)^{-1} Z_{m, *}^{(l)}\right|^{4}\right] \\
& +\frac{C}{\varepsilon^{2}} b_{n}^{-2} \sum_{m} E_{m}\left[\left|\bar{E}_{m}\left[\left(Z_{m, *}^{(l)}\right)^{\top} \partial_{v}\left(S_{m, *}^{(l)}\right)^{-1} Z_{m, *}^{(l)}\right]\right|^{4}\right]
\end{aligned}
$$

We can see that the first term on the right-hand side converges to 0 by Lemma 8.1 and a similar estimate to Point 1 of Lemma 4.3. We show the second term on the right-hand side converges to 0 in probability.

Let $Z_{2, m}^{(0)}$ be similarly defined to $\tilde{Z}_{2, m}$ in (A.4). Let $Z_{1, m, *}^{(l)}=Z_{m}^{(l)}\left(\sigma_{*}\right)-Z_{2, m}^{(l)}$ and $S_{1, m, *}^{(l)}=$ $E_{m}\left[Z_{1, m, *}^{(l)}\left(Z_{1, m, *}^{(l)}\right)^{\top}\right]$. Then Lemmas 8.1 and a similar estimate to (A.5) yield

$$
\begin{aligned}
E_{m}\left[\left|\left(Z_{1, m, *}^{(l)}\right)^{\top} \partial_{v}\left(S_{m, *}^{(l)}\right)^{-1} Z_{2, m}^{(l)}\right|^{4}\right] & \leq C\left|E_{m}\left[\left(\left(Z_{2, m}^{(l)}\right)^{\top} \partial_{v}\left(S_{m, *}^{(l)}\right)^{-1} S_{1, m, *}^{(l)} \partial_{v}\left(S_{m, *}^{(l)}\right)^{-1} Z_{2, m}^{(l)}\right)^{2}\right]\right| \\
& =\bar{R}_{n}\left(b_{n}^{-1} k_{n}^{2}\right),
\end{aligned}
$$

and $E_{m}\left[\left|\left(Z_{1, m, *}^{(l)}\right)^{\top} \partial_{v}\left(S_{m, *}^{(l)}\right)^{-1} Z_{1, m, *}^{(l)}\right|^{4}\right] \leq \bar{R}_{n}\left(b_{n}^{-4}\right) \sum_{j=1}^{4}\left|\operatorname{tr}\left(\left(S_{m, *}^{(l)}\right)^{-1}\right)^{j}\right|=\bar{R}_{n}\left(b_{n}^{-2} k_{n}^{4}\right)$. Moreover, Lemma 8.1 yields

$$
b_{n}^{-2} \sum_{m} E_{m}\left[\bar{E}_{m}\left[\left(Z_{2, m}^{(l)}\right)^{\top} \partial_{v}\left(S_{m, *}^{(l)}\right)^{-1} Z_{2, m}^{(l)}\right]^{4}\right]=\bar{R}_{n}\left(b_{n}^{-2} \ell_{n} k_{n}^{2}\right) \stackrel{p}{\rightarrow} 0,
$$

and therefore, we obtain

$$
\sum_{m=1}^{\left[\ell_{n} t / T\right]} E_{m}\left[\left|\tilde{\mathcal{X}}_{m}^{n}\right|^{2} 1_{\left\{\left|\tilde{\mathcal{X}}_{m}^{n}\right|>\varepsilon\right\}}\right] \stackrel{p}{\rightarrow} 0
$$

Similarly to the proof of Proposition 7.2, we have

$$
\sum_{m=1}^{\left[\ell_{n} t / T\right]} E_{m}\left[\tilde{\mathcal{X}}_{m}^{n}\left(N_{s_{m}}-N_{s_{m-1}}\right)\right] \rightarrow^{p} 0 \quad \text { and } \quad \sum_{m=1}^{\left[\ell_{n} t / T\right]} E_{m}\left[\tilde{\mathcal{X}}_{m}^{n}\left(W_{s_{m}}-W_{s_{m-1}}\right)\right]=0
$$

for any bounded $\mathcal{G}_{t}$-martingale $N$ orthogonal to $\left(W_{t}\right)_{t}$. 
Therefore, by Theorem 3.2 in Jacod [18], it is sufficient to show that

$$
\sum_{m=1}^{\left[\ell_{n} t / T\right]} E_{m}\left[\tilde{\mathcal{X}}_{m}^{n}\left(\tilde{\mathcal{X}}_{m}^{n}\right)^{\top}\right] \stackrel{p}{\rightarrow} \operatorname{diag}\left(-\partial_{\sigma}^{2} \mathcal{Y}_{1}\left(\sigma_{*}, t\right),-\partial_{v}^{2} \mathcal{Y}_{2}\left(v_{*}, t\right)\right),
$$

where $\mathcal{Y}_{2}(v, t)=-\int_{0}^{t} \sum_{j=1}^{2} a_{s}^{j}\left\{\left(v_{j, *} / v_{j}\right)-1+\log \left(v_{j} / v_{j, *}\right)\right\} d s / 2$.

Then we obtain the desired results by

$$
\begin{aligned}
& \sum_{m=1}^{\left[\ell_{n} t / T\right]} E_{m}\left[\tilde{\mathcal{X}}_{m}^{n, 1}\left(\tilde{\mathcal{X}}_{m}^{n, 2}\right)^{\top}\right]=\frac{b_{n}^{-3 / 4}}{2} \sum_{m=1}^{\left[\ell_{n} t / T\right]} \operatorname{tr}\left(\partial_{\sigma} S_{m, *}^{(l)}\left(S_{m, *}^{(l)}\right)^{-1} \partial_{v} S_{m, *}^{(l)}\left(S_{m, *}^{(l)}\right)^{-1}\right) \stackrel{p}{\rightarrow} 0 \\
& \sum_{m=1}^{\left[\ell_{n} t / T\right]} E_{m}\left[\tilde{\mathcal{X}}_{m}^{n, 1}\left(\tilde{\mathcal{X}}_{m}^{n, 1}\right)^{\top}\right] \\
& =\frac{b_{n}^{-1 / 2}}{2} \sum_{m=1}^{\left[\ell_{n} t / T\right]} \operatorname{tr}\left(\left(\partial_{\sigma} S_{m, *}^{(l)}\left(S_{m, *}^{(l)}\right)^{-1}\right)^{2}\right) \\
& \quad=\left.\frac{b_{n}^{-1 / 2}}{2} \sum_{m=1}^{\left[\ell_{n} t / T\right]} E_{m}\left[\partial_{\sigma}^{2}\left\{\left(Z_{m, *}^{(l)}\right)^{\top}\left(S_{m}^{(l)}\right)^{-1} Z_{m, *}^{(l)}+\log \operatorname{det} S_{m}^{(l)}\right\}\right]\right|_{(\sigma, v)=\left(\sigma_{*}, v_{*}\right)} \\
& \quad \stackrel{p}{\rightarrow}-\partial_{\sigma}^{2} \mathcal{Y}_{1}\left(\sigma_{*}, t\right)
\end{aligned}
$$

and similarly

$$
\begin{aligned}
& \sum_{m=1}^{\left[\ell_{n} t / T\right]} E_{m}\left[\tilde{\mathcal{X}}_{m}^{n, 2}\left(\tilde{\mathcal{X}}_{m}^{n, 2}\right)^{\top}\right] \\
& \quad=\left.\frac{b_{n}^{-1}}{2} \sum_{m=1}^{\left[\ell_{n} t / T\right]} E_{m}\left[\partial_{v}^{2}\left\{\left(Z_{m, *}^{(l)}\right)^{\top}\left(S_{m}^{(l)}\right)^{-1} Z_{m, *}^{(l)}+\log \operatorname{det} S_{m}^{(l)}\right\}\right]\right|_{(\sigma, v)=\left(\sigma_{*}, v_{*}\right)} \\
& \quad \stackrel{p}{\rightarrow}-\partial_{v}^{2} \mathcal{Y}_{2}\left(v_{*}, t\right)
\end{aligned}
$$

Proof of Theorem 2.2. Let $\mathbf{U}(u)=\exp \left(u^{\top} \Gamma^{1 / 2} \tilde{\mathcal{N}}-u^{\top} \Gamma u / 2\right)$ for $u \in \mathbb{R}^{d+2}$. Let

$$
Z^{(1)}(\sigma)=\left(\varepsilon_{0}^{n, k},\left(\tilde{Y}_{i}^{k}-\tilde{Y}_{i-1}^{k}-\int_{S_{i-1}^{n, k}}^{S_{i}^{n, k}} \mu^{k}(t, \sigma) d t\right)_{i=1}^{\mathbf{J}_{k, n}}\right)_{k=1,2}
$$

and $S^{(1)}(\sigma, v)$ be a symmetric matrix of size $\mathbf{J}_{1, n}+\mathbf{J}_{2, n}+2$ defined by $\left(S^{(1)}(\sigma, v)\right)_{11}=v_{1}$, $\left(S^{(1)}(\sigma, v)\right)_{\mathbf{J}_{1, n}+2, \mathbf{J}_{1, n}+2}=v_{2}$,

$$
\left(S^{(1)}(\sigma, v)\right)_{i j}=\operatorname{diag}\left(v_{1} M\left(\mathbf{J}_{1, n}+1\right), v_{2} M\left(\mathbf{J}_{2, n}+1\right)\right)_{i j}
$$

$$
\text { if } i \neq j \text { and }\{i, j\} \cap\left\{1, \mathbf{J}_{1, n}+2\right\} \neq \varnothing,
$$




$$
\begin{aligned}
& \left(S^{(1)}(\sigma, v)\right)_{i j}=\int_{S_{i-2}^{n, 1}}^{S_{i-1}^{n, 1}}\left|b_{t}^{1}\right|^{2} d t \delta_{i j}+v_{1} M\left(\mathbf{J}_{1, n}+1\right)_{i j} \quad \text { if } 2 \leq i, j \leq \mathbf{J}_{1, n}+1, \\
& \left(S^{(1)}(\sigma, v)\right)_{i j}=\int_{S_{i^{\prime}-2}^{n, 2}}^{S_{i^{\prime}-1}^{n, 2}}\left|b_{t}^{2}\right|^{2} d t \delta_{i j}+v_{2} M\left(\mathbf{J}_{2, n}+1\right)_{i^{\prime} j^{\prime}} \quad \text { if } 2 \leq i^{\prime}, j^{\prime} \leq \mathbf{J}_{2, n}+1, \\
& \left(S^{(1)}(\sigma, v)\right)_{i j}=\int_{\left[S_{i-2}^{n, 1}, S_{i-1}^{n, 1}\right) \cap\left[S_{j^{\prime}-2}^{n, 2}, S_{j^{\prime}-1}^{n, 2}\right)} b_{t}^{1} \cdot b_{t}^{2} d t \quad \text { if } 2 \leq i \leq \mathbf{J}_{1, n}+1 \text { and } 2 \leq j^{\prime} \leq \mathbf{J}_{2, n}+1,
\end{aligned}
$$

where $i^{\prime}=i-\mathbf{J}_{1, n}-1$ and $j^{\prime}=j-\mathbf{J}_{1, n}-1$. Then we have (8.2) for $l=1$ with

$$
H_{n}^{(1)}(\sigma, v)=-\frac{1}{2}\left(Z^{(1)}(\sigma)\right)^{\top}\left(S^{(1)}(\sigma, v)\right)^{-1} Z^{(1)}(\sigma)-\frac{1}{2} \log \operatorname{det} S^{(1)}(\sigma, v) .
$$

Moreover, Theorem 8.1 and Lemmas 8.2 and 8.3 yield

$$
\begin{aligned}
& \left(H_{n}^{(1)}\left(\sigma_{u^{(1)}}, v_{u^{(1)}}\right)-H_{n}^{(1)}\left(\sigma_{*}, v_{*}\right), \ldots, H_{n}^{(1)}\left(\sigma_{u^{(k)}}, v_{u^{(k)}}\right)-H_{n}^{(1)}\left(\sigma_{*}, v_{*}\right)\right) \\
& \quad \stackrel{d}{\rightarrow}\left(\log \mathbf{U}\left(u^{(1)}\right), \ldots, \log \mathbf{U}\left(u^{(k)}\right)\right)
\end{aligned}
$$

as $n \rightarrow \infty$ for $u^{(1)}, \ldots, u^{(k)} \in \mathbb{R}^{d+2}$.

Furthermore, similar estimates to the proof of Lemma 8.2 yield $\sup _{\sigma, v} \mid b_{n}^{-3 / 4} \partial_{\sigma} \partial_{v} H_{n}^{(1)}(\sigma$, $v)\left|\rightarrow^{p} 0, \sup _{\sigma}\right| b_{n}^{-3 / 4} \partial_{\sigma}^{3} H_{n}^{(1)}\left(\sigma, v_{*}\right) \mid \rightarrow^{p} 0$, and $\sup _{v}\left|b_{n}^{-3 / 2} \partial_{v}^{3} H_{n}^{(1)}\left(\sigma_{*}, v\right)\right| \rightarrow^{p} 0$. Therefore we obtain

$$
H_{n}^{(1)}\left(\sigma_{u}, v_{u}\right)-H_{n}^{(1)}\left(\sigma_{*}, v_{*}\right)-\left(u \cdot \mathbf{V}_{1, n}-u^{\top} \mathbf{V}_{2, n} u / 2\right) \stackrel{p}{\rightarrow} 0
$$

as $n \rightarrow \infty$ for any $u \in \mathbb{R}^{d+2}$, where $\mathbf{V}_{1, n}=\left(b_{n}^{-1 / 4} \partial_{\sigma} H_{n}^{(1)}\left(\sigma_{*}, v_{*}\right), b_{n}^{-1 / 2} \partial_{v} H_{n}^{(1)}\left(\sigma_{*}, v_{*}\right)\right)$ and $\mathbf{V}_{2, n}=-\operatorname{diag}\left(b_{n}^{-1 / 2} \partial_{\sigma}^{2} H_{n}^{(1)}\left(\sigma_{*}, v_{*}\right), b_{n}^{-1} \partial_{v}^{2} H_{n}^{(1)}\left(\sigma_{*}, v_{*}\right)\right)$. (8.4) and (8.5) yield $\mathbf{V}_{1, n} \rightarrow^{d} \Gamma^{1 / 2} \tilde{\mathcal{N}}$ and $\mathbf{V}_{2, n} \rightarrow^{p} \Gamma$, and therefore we obtain the LAN property of the original experiment with $\Gamma_{n}=\mathbf{V}_{2, n}$ and $\mathcal{N}_{n}=\Gamma^{-1 / 2} \mathbf{V}_{1, n}$ by (8.1).

\section{Proof of the results in Section 2.4}

In this final section, we complete the proof of remaining results in Section 2. Proposition 2.2 is proven by the scheme of Yoshida [29,30]. Proposition 6.1 and moment estimates in Lemmas 4.4 and 5.2 enable us to check the assumptions of Theorem 2 in [30]. Then the results on convergence of moments and the Bayes-type estimator are obtained by Proposition 2.2.

Outline of the proof of Proposition 2.2. We apply Theorem 2 in Yoshida [30]. It is sufficient to prove the following five conditions for any $L>0$ with some positive constant $\delta_{1}$ and $\delta_{2}$ :

1. There exists $C_{L}>0$ such that $P\left[\inf _{\sigma \neq \sigma_{*}}\left(-\mathcal{Y}_{1}(\sigma) /\left|\sigma-\sigma_{*}\right|^{2}\right) \leq r^{-1}\right] \leq C_{L} / r^{L}$ and $P\left[\left\{r^{-1}|u|^{2} \leq u^{\top} \Gamma_{1} u / 4 \text { for any } u \in \mathbb{R}^{d}\right\}^{c}\right] \leq C_{L} / r^{L}$ for any $r>0$. 
2. $\sup _{n} E\left[\left(b_{n}^{-1 / 4}\left|\partial_{\sigma} H_{n}\left(\sigma_{*}, \hat{v}_{n}\right)\right|\right)^{L}\right]<\infty$.

3. $\sup _{n} E\left[\left(b_{n}^{\delta_{1}} \sup _{\sigma}\left|b_{n}^{-1 / 2}\left(H_{n}\left(\sigma, \hat{v}_{n}\right)-H_{n}\left(\sigma_{*}, \hat{v}_{n}\right)\right)-\mathcal{Y}_{1}(\sigma)\right|\right)^{L}\right]<\infty$.

4. $\sup _{n} E\left[\left(b_{n}^{-1 / 2} \sup _{\sigma}\left|\partial_{\sigma}^{3} H_{n}\left(\sigma, \hat{v}_{n}\right)\right|\right)^{L}\right]<\infty$.

5. $\sup _{n} E\left[\left(b_{n}^{\delta_{2}}\left|b_{n}^{-1 / 2} \partial_{\sigma}^{2} H_{n}\left(\sigma_{*}, \hat{v}_{n}\right)+\Gamma_{1}\right|\right)^{L}\right]<\infty$.

By Taylor's formula for $\mathcal{Y}_{1}(\sigma)$ and relations $\mathcal{Y}_{1}\left(\sigma_{*}\right)=\partial_{\sigma} \mathcal{Y}_{1}\left(\sigma_{*}\right)=0$, we obtain $\inf _{\sigma \neq \sigma_{*}}\left(-\mathcal{Y}_{1}(\sigma) /\left|\sigma-\sigma_{*}\right|^{2}\right) \leq \inf _{u \in \mathbb{R}^{d} \backslash\{0\}} u^{\top} \Gamma_{1} u /\left(2|u|^{2}\right)$. Then (B3) and the proof of Proposition 6.1 yield point 1. By Lemmas 4.4 and 5.2, Remark 5.2, and a similar argument to the proof of Proposition 2.1, we obtain 3-5 and $\sup _{n} E\left[\left(b_{n}^{-1 / 4}\left|\partial_{\sigma} H_{n}\left(\sigma_{*}, \hat{v}_{n}\right)-\partial_{\sigma} \tilde{H}_{n}\left(\sigma_{*}, v_{*}\right)\right|\right)^{L}\right]<\infty$. Moreover, by the Burkholder-Davis-Gundy inequality, we obtain

$$
\begin{aligned}
E[ & \left.\left|b_{n}^{-1 / 4} \partial_{\sigma} \tilde{H}_{n}\left(\sigma_{*}, v_{*}\right)\right|^{L}\right] \\
= & E\left[\left|\frac{b_{n}^{-1 / 4}}{2} \sum_{m} \bar{E}_{m}\left[\tilde{Z}_{m}^{\top} \partial_{\sigma} \tilde{S}_{m, *}^{-1} \tilde{Z}_{m}\right]\right|^{L}\right] \leq C E\left[\left|b_{n}^{-\frac{1}{2}} \sum_{m} \bar{E}_{m}\left[\tilde{Z}_{m}^{\top} \partial_{\sigma} \tilde{S}_{m, *}^{-1} \tilde{Z}_{m}\right]^{2}\right|^{L / 2}\right] \\
\leq & C E\left[\left|b_{n}^{-\frac{1}{2}} \sum_{m} E_{m}\left[\bar{E}_{m}\left[\tilde{Z}_{m}^{\top} \partial_{\sigma} \tilde{S}_{m, *}^{-1} \tilde{Z}_{m}\right]^{2}\right]\right|^{L / 2}\right] \\
& +C E\left[\left|b_{n}^{-1} \sum_{m} \bar{E}_{m}\left[\bar{E}_{m}\left[\tilde{Z}_{m}^{\top} \partial_{\sigma} \tilde{S}_{m, *}^{-1} \tilde{Z}_{m}\right]^{2}\right]^{2}\right|^{L / 4}\right] \\
\leq & C E\left[\left|b_{n}^{-\frac{1}{2}} \sum_{m} \operatorname{tr}\left(\left(\partial_{\sigma} \tilde{S}_{m, *}^{-1} \tilde{S}_{m, *}\right)^{2}\right)\right|^{L / 2}\right] \\
& +C E\left[\left|b_{n}^{-1} \sum_{m} E_{m}\left[\left(\tilde{Z}_{m}^{\top} \partial_{\sigma} \tilde{S}_{m, *}^{-1} \tilde{Z}_{m}\right)^{L}\right]^{4 / L}\right|^{L / 4}\right]+O(1) \\
= & O\left(\left(b_{n}^{-1 / 2} \ell_{n} b_{n}^{1 / 2} \ell_{n}^{-1}\right)^{L / 2}\right)+E\left[\bar{R}_{n}\left(\left(b_{n}^{-5} k_{n}^{8} \ell_{n}\right)^{L / 4}\right)\right]+O(1) \\
= & O(1)
\end{aligned}
$$

for $L \geq 4$, which implies point 2 .

Proof of Theorem 2.3. We extend $\mathbf{Z}_{n}(u)$ to a continuous function on $\mathbb{R}^{d}$ satisfying $\lim _{|u| \rightarrow \infty} \mathbf{Z}_{n}(u)=0$ with the supremum norm of the extended function the same as for the original one. Then by Theorem 5 and Remark 5 in Yoshida [30], it is sufficient to show $\limsup _{n \rightarrow \infty} E\left[\left|b_{n}^{1 / 4}\left(\hat{\sigma}_{n}-\sigma_{*}\right)\right|^{p}\right]<\infty$ for any $p>0$ and $\mathbf{Z}_{n} \rightarrow^{s-\mathcal{L}} \mathbf{Z}$ in $C(B(R))$ as $n \rightarrow \infty$ for any $R>0$, where $\mathbf{Z}(u)=\exp \left(\mathcal{N} \cdot u-u^{\top} \Gamma_{1} u / 2\right)$ and $B(R)=\{u ;|u| \leq R\}$.

By Lemma 4.4 and a similar argument to the proof of Proposition 2.1, we have

$$
\lim _{N \rightarrow \infty} \sup _{n \geq N} E\left[\sup _{u \in B(R)}\left|\partial_{u} \log \mathbf{Z}_{n}(u)\right|\right]<\infty .
$$

Then Propositions 2.1 and 7.2 and tightness criterion in $C$ space in Billingsley [6] yield $\log \mathbf{Z}_{n} \rightarrow^{s-\mathcal{L}} \log \mathbf{Z}$ in $C(B(R))$. Then (2.12) completes the proof. 
Proof of Theorem 2.4. By Theorem 10 in Yoshida [30], it is sufficient to show

$$
\sup _{n} E\left[\left(\int_{U_{n}} \mathbf{Z}_{n}(u) \pi\left(\sigma_{*}+b_{n}^{-1 / 4} u\right) d u\right)^{-1}\right]<\infty .
$$

Similarly to the proof of Proposition 2.2, there exists $\delta>0$ such that $\sup _{n} E\left[\mid H_{n}\left(\sigma_{*}+\right.\right.$ $\left.\left.b_{n}^{-1 / 4} u, \hat{v}_{n}\right)-\left.H_{n}\left(\sigma_{*}, \hat{v}_{n}\right)\right|^{p}\right] \leq C_{p}|u|^{p}$ for any $u \in U(\delta)$, where $U(\delta)=\left\{u \in \mathbb{R}^{d} ;\left|u_{i}\right| \leq \delta(i=\right.$ $1, \ldots, d)\}$. Then we have $(9.1)$ by Lemma 2 in [30].

\section{Appendix}

\section{A.1. Results from linear algebra}

Lemma A.1. Let $A$ and $B$ be matrices, with A nonnegative definite and symmetric. Then

$$
|\operatorname{tr}(A B)| \leq \operatorname{tr}(A)\|B\|
$$

Lemma A.2. Let $l \in \mathbb{N}, A^{j}$ and $B^{j}$ be real-valued matrices and $\left\{\lambda_{k}^{j}\right\}_{k}$ be eigenvalues of $A^{j}$ for $1 \leq j \leq l$. Assume that $A^{j}$ is symmetric and all the elements of $B^{j}$ are nonnegative for $1 \leq j \leq l$. Then

$$
\sum_{i_{1}, \ldots, i_{2 l}} \prod_{j=1}^{l}\left(\left|A_{i_{2 j-1}, i_{2 j}}^{j}\right| B_{i_{2 j}, i_{2 j+1}}^{j}\right) \leq \prod_{j=1}^{l}\left(\left\|B^{j}\right\| \sum_{k}\left|\lambda_{k}^{j}\right|\right),
$$

where $i_{2 l+1}=i_{1}$.

Proof. Let $U^{j}$ be an orthogonal matrix such that $A^{j}=\left(U^{j}\right)^{\top} \operatorname{diag}\left(\left(\lambda_{k}^{j}\right)_{k}\right) U^{j}$. Then

$$
\begin{aligned}
\sum_{i_{1}, \ldots, i_{2 l} l} \prod_{j=1}^{l}\left(\left|A_{i_{2 j-1}, i_{2 j}}^{j}\right| B_{i_{2 j}, i_{2 j+1}}^{j}\right) & \leq \sum_{k_{1}, \ldots, k_{l}} \sum_{i_{1}, \ldots, i_{2 l}} \prod_{j=1}^{l}\left(\left|\lambda_{k_{j}}^{j}\left\|U_{k_{j}, i_{2 j-1}}^{j}\right\| U_{k_{j}, i_{2 j}}^{j}\right| B_{i_{2 j}, i_{2 j+1}}^{j}\right) \\
& \leq \sum_{k_{1}, \ldots, k_{l}} \prod_{j=1}^{l}\left\{\left|\lambda_{k_{j}}^{j}\right|\left\|B^{j}\right\| \sum_{i}\left(U_{k_{j}, i}^{j}\right)^{2}\right\} \\
& =\prod_{j=1}^{l}\left(\left\|B^{j}\right\| \sum_{k}\left|\lambda_{k}^{j}\right|\right) .
\end{aligned}
$$

Lemma A.3. Let $A$ be a symmetric matrix with $\|A\|<1$. Then $\log \operatorname{det}(\mathcal{E}+A)=$ $\sum_{p=1}^{\infty}(-1)^{p-1} p^{-1} \operatorname{tr}\left(A^{p}\right)$. 
Proof. Let $\left\{\lambda_{j}\right\}_{j=1}^{k}$ be eigenvalues of $A$. Then $\sup _{j}\left|\lambda_{j}\right|=\|A\|<1$, and hence

$$
\log \operatorname{det}(\mathcal{E}+A)=\sum_{j} \log \left(1+\lambda_{j}\right)=\sum_{j} \sum_{p=1}^{\infty}(-1)^{p-1} p^{-1} \lambda_{j}^{p}=\sum_{p=1}^{\infty}(-1)^{p-1} p^{-1} \operatorname{tr}\left(A^{p}\right) .
$$

Lemma A.4. Let $A$ and $B$ be symmetric, positive definite matrices. Assume that $v^{\top} A v \geq v^{\top} B v$ for any vector $v$. Then $\left\|A^{-1}\right\| \leq\left\|B^{-1}\right\|$ and $\left\|A^{-1 / 2}\right\| \leq\left\|B^{-1 / 2}\right\|$.

Proof. Let $\left(\lambda_{j}^{A}\right)_{j}$ and $\left(\lambda_{j}^{B}\right)_{j}$ be eigenvalues of $A$ and $B$, respectively. Then for any unit vector $v$, there exists an orthogonal matrix $U$ such that

$$
\sum_{j} \lambda_{j}^{A} v_{j}^{2} \geq \sum_{j} \lambda_{j}^{B}(U v)_{j}^{2} \geq \inf _{j} \lambda_{j}^{B}
$$

Therefore, we obtain $\left\|A^{-1}\right\|^{-1}=\inf _{j} \lambda_{j}^{A} \geq \inf _{j} \lambda_{j}^{B}=\left\|B^{-1}\right\|^{-1}$ and $\left\|A^{-1 / 2}\right\|^{-1}=\inf _{j}\left(\lambda_{j}^{A}\right)^{1 / 2} \geq$ $\inf _{j}\left(\lambda_{j}^{B}\right)^{1 / 2}=\left\|B^{-1 / 2}\right\|^{-1}$.

Lemma A.5. Let $B$ be a symmetric, positive definite matrix and $A$ be a symmetric, nonnegative definite matrix. Then $\operatorname{tr}(A B) \geq \operatorname{tr}(A)\left\|B^{-1}\right\|^{-1}$.

Proof. Let $\left\{\lambda_{j}^{A}\right\}_{j}$ and $\left\{\lambda_{j}^{B}\right\}_{j}$ be eigenvalues of $A$ and $B$, respectively, and $U$ be an orthogonal matrix satisfying $U A U^{\top}=\operatorname{diag}\left(\left(\lambda_{j}^{A}\right)_{j}\right)$. Then since $\left(U B U^{\top}\right)_{j j} \geq \inf _{j} \lambda_{j}^{B}=\left\|B^{-1}\right\|^{-1}$, we obtain

$$
\operatorname{tr}(A B)=\sum_{j} \lambda_{j}^{A}\left(U B U^{\top}\right)_{j j} \geq \sum_{j} \lambda_{j}^{A}\left\|B^{-1}\right\|^{-1}=\operatorname{tr}(A)\left\|B^{-1}\right\|^{-1} .
$$

Lemma A.6. Let $\eta>0$ and $A$ be a symmetric matrix. Assume that $\mathcal{E}+A$ is positive definite and $\|\mathcal{E}+A\| \leq \eta$. Then $\operatorname{tr}(A)-\log \operatorname{det}(\mathcal{E}+A) \geq \operatorname{tr}\left(A^{2}\right) /(2(\eta \vee 1))$.

Proof. We easily obtain the desired results by using the fact that $\log \operatorname{det}(\mathcal{E}+A)=\sum_{k} \log \left(1+\lambda_{k}\right)$ and that $x-x^{2} /(2(\eta \vee 1)) \geq \log (1+x)$ for $-1<x \leq \eta-1$, where $\left(\lambda_{j}\right)_{j}$ are eigenvalues of $A$.

Lemma A.7. Let $A$ be a symmetric matrix, $B$ a matrix of suitable size and $\left(\lambda_{j}\right)_{j}$ eigenvalues of $B^{\top} A B$. Then

1. $\left|\left(B^{\top} A B\right)_{i i}\right| \leq\|A\|\left(B^{\top} B\right)_{i i}$ for any $i$.

2. $\sum_{j}\left|\lambda_{j}\right| \leq\|A\| \operatorname{tr}\left(B^{\top} B\right)$.

Proof. 1. Let $U$ be an orthogonal matrix and let $\left\{\lambda_{j}^{\prime}\right\}_{j}$ be eigenvalues of $A$ such that $U^{\top} A U=$ $\operatorname{diag}\left(\left(\lambda_{j}^{\prime}\right)_{j}\right)$. Then we obtain

$$
\left|\left(B^{\top} A B\right)_{i i}\right|=\left|\sum_{j} \lambda_{j}^{\prime}\left(\left(U^{\top} B\right)_{j i}\right)^{2}\right| \leq\|A\| \sum_{j}\left(\left(U^{\top} B\right)_{j i}\right)^{2}=\|A\|\left(B^{\top} B\right)_{i i} .
$$


2. There exists an orthogonal matrix $V$ such that $\lambda_{j}=\left(V^{\top} B^{\top} A B V\right)_{j j}$ for any $j$. Then

$$
\sum_{j}\left|\lambda_{j}\right|=\sum_{j}\left|\left(V^{\top} B^{\top} A B V\right)_{j j}\right| \leq\|A\| \sum_{j}\left(V^{\top} B^{\top} B V\right)_{j j}=\|A\| \operatorname{tr}\left(B^{\top} B\right)
$$

by 1 .

\section{A.2. Proof of (2.10)}

Proof of (2.10). Let $\tilde{a}_{t}^{3}=\sqrt{\tilde{a}_{t}^{1} \tilde{a}_{t}^{2}}, B_{t}=\sqrt{\operatorname{det}\left(b_{t} b_{t}^{\top}\right)}, \Upsilon_{t}=\left(\tilde{a}_{t}^{1}\left|b_{t}^{1}\right|^{2}+\tilde{a}_{t}^{2}\left|b_{t}^{2}\right|+2 \tilde{a}_{t}^{3} B_{t}\right)^{1 / 2}$, and $\psi(x, y)=\left(x y^{\top}+y x^{\top}\right) / 2$ for vectors $x, y$. Then (2.8) yields

$$
\begin{aligned}
\mathcal{Y}_{1}(\sigma)= & \int_{0}^{T}\left\{\frac{\sum_{j=1}^{2}\left(\left|b_{t}^{j}\right|^{2}-\left|b_{t, *}^{j}\right|^{2}\right)\left(\tilde{a}_{t}^{3}\left|b_{t}^{3-j}\right|^{2}+\tilde{a}_{t}^{j} B_{t}\right)-2 \tilde{a}_{t}^{3}\left(b_{t}^{1} \cdot b_{t}^{2}-b_{t, *}^{1} \cdot b_{t, *}^{2}\right) b_{t}^{1} \cdot b_{t}^{2}}{4 B_{t} \Upsilon_{t}}\right. \\
& \left.-\frac{\Upsilon_{t}-\Upsilon_{t}^{(0)}}{2}\right\} d t .
\end{aligned}
$$

Hence we obtain

$$
\begin{aligned}
\partial_{\sigma}^{2} \mathcal{Y}_{1}\left(\sigma_{*}\right)= & \int_{0}^{T}\left\{\left.\frac{\sum_{j=1}^{2} \partial_{\sigma}^{2}\left|b_{t}^{j}\right|^{2}\left(\tilde{a}_{t}^{3}\left|b_{t}^{3-j}\right|^{2}+\tilde{a}_{t}^{j} B_{t}\right)-2 \tilde{a}_{t}^{3} \partial_{\sigma}^{2} b_{t}^{1} \cdot b_{t}^{2} b_{t}^{1} \cdot b_{t}^{2}}{4 B_{t} \Upsilon_{t}}\right|_{\sigma=\sigma_{*}}\right. \\
& +\left.\sum_{j=1}^{2} \psi\left(\partial_{\sigma}\left|b_{t}^{j}\right|^{2}, \partial_{\sigma} \frac{\tilde{a}_{t}^{3}\left|b_{t}^{3-j}\right|^{2}+\tilde{a}_{t}^{j} B_{t}}{2 B_{t} \Upsilon_{t}}\right)\right|_{\sigma=\sigma_{*}}-\left.\psi\left(\partial_{\sigma} b_{t}^{1} \cdot b_{t}^{2}, \tilde{a}_{t}^{3} \partial_{\sigma} \frac{b_{t}^{1} \cdot b_{t}^{2}}{B_{t} \Upsilon_{t}}\right)\right|_{\sigma=\sigma_{*}} \\
& \left.-\left.\frac{\partial_{\sigma}^{2} \Upsilon_{t}^{2}}{4 \Upsilon_{t}}\right|_{\sigma=\sigma_{*}}+\frac{\Upsilon_{t}^{(1)}\left(\Upsilon_{t}^{(1)}\right)^{\top}}{2 \Upsilon_{t}^{(0)}}\right\} d t .
\end{aligned}
$$

Terms involving $\partial_{\sigma}^{2}$ in the integrand of right-hand side are rewritten as

$$
\begin{aligned}
& \left.\left(\tilde{a}_{t}^{3} \frac{\sum_{j=1}^{2} \partial_{\sigma}^{2}\left|b_{t}^{j}\right|^{2}\left|b_{t}^{3-j}\right|^{2}-2 \partial_{\sigma}^{2} b_{t}^{1} \cdot b_{t}^{2} b_{t}^{1} \cdot b_{t}^{2}}{4 B_{t} \Upsilon_{t}}-\tilde{a}_{t}^{3} \frac{2 \partial_{\sigma}^{2} B_{t}}{4 \Upsilon_{t}}\right)\right|_{\sigma=\sigma_{*}} \\
& \quad=\left.\frac{\tilde{a}_{t}^{3}}{4 \Upsilon_{t}^{(0)}}\left\{\frac{\partial_{\sigma}^{2}\left|b_{t}^{1}\right|^{2}\left|b_{t}^{2}\right|^{2}+\left|b_{t}^{1}\right|^{2} \partial_{\sigma}^{2}\left|b_{t}^{2}\right|^{2}-2 b_{t}^{1} \cdot b_{t}^{2} \partial_{\sigma}^{2} b_{t}^{1} \cdot b_{t}^{2}}{B_{t}}-\frac{\partial_{\sigma}^{2} B_{t}^{2}}{B_{t}}+\frac{2 B_{4, t}^{(1)}\left(B_{4, t}^{(1)}\right)^{\top}}{B_{4, t}^{(0)}}\right\}\right|_{\sigma=\sigma_{*}} \\
& \quad=\frac{\tilde{a}_{t}^{3}}{4 \Upsilon_{t}^{(0)}}\left\{-\frac{2 \psi\left(B_{1, t}^{(1)}, B_{2, t}^{(1)}\right)-2 B_{3, t}^{(1)}\left(B_{3, t}^{(1)}\right)^{\top}}{B_{4, t}^{(0)}}+\frac{2 B_{4, t}^{(1)}\left(B_{4, t}^{(1)}\right)^{\top}}{B_{4, t}^{(0)}}\right\} .
\end{aligned}
$$


Other terms are rewritten as

$$
\begin{aligned}
\sum_{j=1}^{2} \psi & \left.\left(\partial_{\sigma}\left|b_{t}^{j}\right|^{2}, \partial_{\sigma} \frac{\tilde{a}_{t}^{3}\left|b_{t}^{3-j}\right|^{2}+\tilde{a}_{t}^{j} B_{t}}{2 B_{t} \Upsilon_{t}}\right)\right|_{\sigma=\sigma_{*}}-\left.\psi\left(\partial_{\sigma} b_{t}^{1} \cdot b_{t}^{2}, \tilde{a}_{t}^{3} \partial_{\sigma} \frac{b_{t}^{1} \cdot b_{t}^{2}}{B_{t} \Upsilon_{t}}\right)\right|_{\sigma=\sigma_{*}} \\
& +\frac{\Upsilon_{t}^{(1)}\left(\Upsilon_{t}^{(1)}\right)^{\top}}{2 \Upsilon_{t}^{(0)}} \\
= & \sum_{j=1}^{2} \psi\left(B_{j, t}^{(1)}, \frac{\tilde{a}_{t}^{3} B_{3-j, t}^{(1)}}{2 B_{4, t}^{(0)} \Upsilon_{t}^{(0)}}-\frac{\tilde{a}_{t}^{3} B_{3-j, t}^{(0)} B_{4, t}^{(1)}}{2\left(B_{4, t}^{(0)}\right)^{2} \Upsilon_{t}^{(0)}}-\frac{\left(\tilde{a}_{t}^{3} B_{3-j, t}^{(0)}+\tilde{a}_{t}^{j} B_{4, t}^{(0)}\right) \Upsilon_{t}^{(1)}}{2 B_{4, t}^{(0)}\left(\Upsilon_{t}^{(0)}\right)^{2}}\right) \\
& -\tilde{a}_{t}^{3} \psi\left(B_{3, t}^{(1)}, \frac{B_{3, t}^{(1)}}{B_{4, t}^{(0)} \Upsilon_{t}^{(0)}}-\frac{B_{3, t}^{(0)} B_{4, t}^{(1)}}{\left(B_{4, t}^{(0)}\right)^{2} \Upsilon_{t}^{(0)}}-\frac{B_{3, t}^{(0)} \Upsilon_{t}^{(1)}}{B_{4, t}^{(0)}\left(\Upsilon_{t}^{(0)}\right)^{2}}\right)+\frac{\Upsilon_{t}^{(1)}\left(\Upsilon_{t}^{(1)}\right)^{\top}}{2 \Upsilon_{t}^{(0)}} \\
= & -\tilde{a}_{t}^{3} \psi\left(B_{1, t}^{(1)} B_{2, t}^{(0)}+B_{2, t}^{(1)} B_{1, t}^{(0)}-2 B_{3, t}^{(0)} B_{3, t}^{(1)}, \frac{B_{4, t}^{(1)}}{2\left(B_{4, t}^{(0)}\right)^{2} \Upsilon_{t}^{(0)}}+\frac{\Upsilon_{t}^{(1)}}{2 B_{4, t}^{(0)}\left(\Upsilon_{t}^{(0)}\right)^{2}}\right) \\
& +\tilde{a}_{t}^{3} \frac{\psi\left(B_{1, t}^{(1)}, B_{2, t}^{(1)}\right)-B_{3, t}^{(1)}\left(B_{3, t}^{(1)}\right)^{\top}}{B_{4, t}^{(0)} \Upsilon_{t}^{(0)}}-\psi\left(\tilde{a}_{t}^{1} B_{1, t}^{(1)}+\tilde{a}_{t}^{2} B_{2, t}^{(1)}, \frac{\Upsilon_{t}^{(1)}}{2\left(\Upsilon_{t}^{(0)}\right)^{2}}\right)+\frac{\Upsilon_{t}^{(1)}\left(\Upsilon_{t}^{(1)}\right)^{\top}}{2 \Upsilon_{t}^{(0)}} \\
= & \tilde{a}_{t}^{3} \frac{\psi\left(B_{1, t}^{(1)}, B_{2, t}^{(1)}\right)-B_{3, t}^{(1)}\left(B_{3, t}^{(1)}\right)^{\top}}{B_{4, t}^{(0)} \Upsilon_{t}^{(0)}}-\frac{\tilde{a}_{t}^{3} B_{4, t}^{(1)}\left(B_{4, t}^{(1)}\right)^{\top}}{B_{4, t}^{(0)} \Upsilon_{t}^{(0)}}-\frac{\Upsilon_{t}^{(1)}\left(\Upsilon_{t}^{(1)}\right)^{\top}}{2 \Upsilon_{t}^{(0)}}
\end{aligned}
$$

Here we have used equations $B_{1, t}^{(1)} B_{2, t}^{(0)}+B_{2, t}^{(1)} B_{1, t}^{(0)}-2 B_{3, t}^{(0)} B_{3, t}^{(1)}=2 B_{4, t}^{(0)} B_{4, t}^{(1)}$ and $\tilde{a}_{t}^{1} B_{1, t}^{(1)}+$ $\tilde{a}_{t}^{2} B_{2, t}^{(1)}+2 \tilde{a}_{t}^{3} B_{4, t}^{(1)}=2 \Upsilon_{t}^{(0)} \Upsilon_{t}^{(1)}$. Therefore, we obtain (2.10).

\section{A.3. Proof of Lemma 4.3}

Let $\mathbf{A}_{m}$ be a $\left(k_{m}^{1}+k_{m}^{2}\right) \times\left(k_{m}^{1}+k_{m}^{2}\right)$ matrix with elements $\left(\mathbf{A}_{m}\right)_{i j}=1_{i \geq j} 1_{\left\{i \leq k_{m}^{1} \text { or } j>k_{m}^{1}\right\}}$, let $\mathbf{1}$ be a matrix with all elements equal to 1 and $M_{m, *}=M_{m}\left(v_{*}\right)$.

Lemma A.8. Let $n \in \mathbb{N}, 1 \leq m \leq \ell_{n}, q, q^{\prime} \in \mathbb{N}$ such that $q^{\prime} \leq 2 q, A_{m}:\left\{1, \ldots, k_{m}^{1}+k_{m}^{2}\right\}^{q^{\prime}} \rightarrow$ $\{0,1\}$ be a random map, $\iota:\left\{1, \ldots, q^{\prime}\right\} \rightarrow\{1, \ldots, 2 q\}$ be an injection, and $\mathbf{S}$ be a symmetric, $\mathcal{G}_{s_{m-1}}$-measurable random matrix of size $k_{m}^{1}+k_{m}^{2}$ satisfying $\left\|M_{m, *} \mathbf{S} M_{m, *}\right\| \leq b_{n}^{-1}$. Let $\mathcal{K}=$ $\sum_{j_{1}, \ldots, j_{q^{\prime}}=1}^{k_{m}^{1}+k_{m}^{2}} A_{m}\left(j_{1}, \ldots, j_{q^{\prime}}\right)$. Then there exists a positive constant $C$ that depends only on $v_{*}$ and $q$ such that

$$
\left|\sum_{i_{1}, \ldots, i_{2 q}} \prod_{j=1}^{q}\left(\left(\mathbf{A}_{m}^{\top}\right)^{-1} \mathbf{S A}_{m}^{-1}\right)_{i_{2 j-1}, i_{2 j}} A_{m}\left(i_{l(1)}, \ldots, i_{l\left(q^{\prime}\right)}\right)\right| \leq C \mathcal{K}\left(\bar{k}_{n} / \underline{k}_{n}\right)^{2 q} b_{n}^{-q} \bar{k}_{n}^{q+q^{\prime}-2\left[q^{\prime} / 2\right]} .
$$


Proof. Let $\hat{\mathbf{S}}=\left(\mathbf{A}_{m}^{\top}\right)^{-1} \mathbf{S} \mathbf{A}_{m}^{-1}$ and $\tilde{\mathbf{A}}_{m}=M_{m, *} \mathbf{A}_{m}^{\top}$. Then a simple calculation shows that

$$
\left(\tilde{\mathbf{A}}_{m}^{-1}\right)_{i, j}= \begin{cases}\left(k_{m}^{\mathbf{k}(i)}+1\right)^{-1}\left(j-k_{m}^{1} 1_{\{\mathbf{k}(i)=2\}}\right) v_{\mathbf{k}(i), *}^{-1}, & \mathbf{k}(i)=\mathbf{k}(j) \text { and } i \geq j, \\ -\left(k_{m}^{\mathbf{k}(i)}+1\right)^{-1}\left(k_{m}^{\mathbf{k}(i)}-j+1+k_{m}^{1} 1_{\{\mathbf{k}(i)=2\}}\right) v_{\mathbf{k}(i), *}^{-1}, & \mathbf{k}(i)=\mathbf{k}(j) \text { and } i<j, \\ 0, & \text { otherwise },\end{cases}
$$

where $\{\mathbf{k}(i)\}_{i=1}^{k_{m}^{1}+k_{m}^{2}}$ is defined by $\mathbf{k}(i)=1$ for $1 \leq i \leq k_{m}^{1}$ and $\mathbf{k}(i)=2$ for $k_{m}^{1}<i \leq k_{m}^{1}+k_{m}^{2}$. Therefore, we have

$$
\begin{aligned}
\left|\hat{\mathbf{S}}_{i j}\right| & =\left|\left(\tilde{\mathbf{A}}_{m}^{-1} M_{m, *} \mathbf{S} M_{m, *}\left(\tilde{\mathbf{A}}_{m}^{\top}\right)^{-1}\right)_{i j}\right| \\
& \leq\left(\sum_{k}\left(\left(\tilde{\mathbf{A}}_{m}^{-1}\right)_{i, k}\right)^{2}\right)^{1 / 2}\left\|M_{m, *} \mathbf{S} M_{m, *}\right\|\left(\sum_{k}\left(\left(\tilde{\mathbf{A}}_{m}^{-1}\right)_{j, k}\right)^{2}\right)^{1 / 2} \\
& \leq\left(v_{1, *}^{-1}+v_{2, *}^{-1}\right) b_{n}^{-1} \bar{k}_{n} .
\end{aligned}
$$

Similarly, we have

$$
\left|(\hat{\mathbf{S}} \mathbf{1} \hat{\mathbf{S}})_{i j}\right| \leq\left(\max _{i} \sum_{k}\left(\tilde{\mathbf{A}}_{m}^{-1}\right)_{i k}^{2}\right)\left\|M_{m, *} \mathbf{S} M_{m, *}\right\|^{2}\left\|\left(\tilde{\mathbf{A}}_{m}^{-1}\right)^{\top} \mathbf{1} \tilde{\mathbf{A}}_{m}^{-1}\right\| \leq C b_{n}^{-2} \bar{k}_{n}^{2}
$$

Moreover, since $\sum_{i}\left(\left(\mathbf{A}_{m} M_{m, *} \mathbf{A}_{m}^{\top}\right)^{-1}\right)_{i j}=v_{\mathbf{k}(j), *}^{-1} /\left(k_{m}^{j}+1\right)$, we obtain

$$
\begin{aligned}
|\operatorname{tr}(\hat{\mathbf{S}} \mathbf{1})| & \leq\left\|M_{m, *} \mathbf{S} M_{m, *}\right\| \sum_{i, j, k, l}\left(\left(\mathbf{A}_{m} M_{m, *} \mathbf{A}_{m}^{\top}\right)^{-1}\right)_{i k}\left(\mathbf{A}_{m} \mathbf{A}_{m}^{\top}\right)_{k l}\left(\left(\mathbf{A}_{m} M_{m, *} \mathbf{A}_{m}^{\top}\right)^{-1}\right)_{l j} \\
& \leq C \underline{\mathrm{k}}_{n}^{-2} b_{n}^{-1} \sum_{k, l}\left(\mathbf{A}_{m} \mathbf{A}_{m}^{\top}\right)_{k l} \leq C \underline{\mathrm{k}}_{n}^{-2} b_{n}^{-1} \bar{k}_{n}^{3} .
\end{aligned}
$$

If both $i_{2 j-1}$ and $i_{2 j}$ are outside the image of $\iota$, we have

$$
\sum_{i_{2 j-1}, i_{2 j}} \hat{\mathbf{S}}_{i_{2 j-1}, i_{2 j}} A_{m}\left(i_{\iota(1)}, \ldots, i_{l\left(q^{\prime}\right)}\right)=\operatorname{tr}(\hat{\mathbf{S}} \mathbf{1}) A_{m}\left(i_{\iota(1)}, \ldots, i_{l\left(q^{\prime}\right)}\right) .
$$

Moreover, if both $i_{2 j-1}$ and $i_{2 k-1}$ are in the image of $\iota$ and neither $i_{2 j}$ nor $i_{2 k}$ is in it, then we have

$$
\begin{gathered}
\sum_{i_{2 j}, i_{2 k}} \hat{\mathbf{S}}_{i_{2 j-1}, i_{2 j}} \hat{\mathbf{S}}_{i_{2 k-1}, i_{2 k}} A_{m}\left(i_{l(1)}, \ldots, i_{l\left(q^{\prime}\right)}\right) \\
=(\hat{\mathbf{S}} \mathbf{1})_{i_{2 j-1}, i_{2 k-1}} A_{m}\left(i_{l(1)}, \ldots, i_{l\left(q^{\prime}\right)}\right)
\end{gathered}
$$


Therefore there exist $\alpha_{k} \in\{0,1\}$ for $1 \leq k \leq\left[q^{\prime} / 2\right], 0 \leq s \leq\left[\left(2 q-q^{\prime}\right) / 2\right]$ and a bijection $\iota^{\prime}$ : $\left\{1, \ldots, q^{\prime}\right\} \rightarrow\left\{1, \ldots, q^{\prime}\right\}$ such that $\sum_{k=1}^{\left[q^{\prime} / 2\right]} \alpha_{k}+\left[q^{\prime} / 2\right]+s=q-\left(q^{\prime}-2\left[q^{\prime} / 2\right]\right)$ and

$$
\begin{aligned}
& \left|\sum_{i_{1}, \ldots, i_{2 q}} \prod_{j=1}^{q} \hat{\mathbf{S}}_{i_{2 j-1}, i_{2 j}} A_{m}\left(i_{l(1)}, \ldots, i_{l\left(q^{\prime}\right)}\right)\right| \\
& \quad \leq\left(C b_{n}^{-1} \bar{k}_{n}^{2}\right)^{q^{\prime}-2\left[q^{\prime} / 2\right]}\left|\sum_{j_{1}, \ldots, j_{q^{\prime}}} \prod_{k=1}^{\left[q^{\prime} / 2\right]}\right|\left((\hat{\mathbf{S}} \mathbf{1})^{\alpha_{k}} \hat{\mathbf{S}}\right)_{j_{\iota^{\prime}(2 k-1)}, j_{\iota^{\prime}(2 k)}}\left|\operatorname{tr}(\hat{\mathbf{S}} \mathbf{1})^{s} A_{m}\left(j_{1}, \ldots, j_{q^{\prime}}\right)\right| \\
& \quad \leq C\left(b_{n}^{-1} \bar{k}_{n}^{2}\right)^{q^{\prime}-2\left[q^{\prime} / 2\right]}\left(\bar{k}_{n} / \underline{\mathbf{k}}_{n}\right)^{2 q}\left(b_{n}^{-1} \bar{k}_{n}\right)^{q-\left(q^{\prime}-2\left[q^{\prime} / 2\right]\right)} \mathcal{K} \leq C \mathcal{K}\left(\bar{k}_{n} / \underline{\mathbf{k}}_{n}\right)^{2 q} b_{n}^{-q} \bar{k}_{n}^{q+q^{\prime}-2\left[q^{\prime} / 2\right]},
\end{aligned}
$$

by (A.1)-(A.3).

Proof of Lemma 4.3. Let $\left\{\tilde{\varepsilon}_{i, m}\right\}_{1 \leq i \leq k_{m}^{1}+k_{m}^{2}}$ and $\left\{\dot{\varepsilon}_{i, m}\right\}_{1 \leq i \leq k_{m}^{1}+k_{m}^{2}}$ be sequences of random variables defined by $\tilde{\varepsilon}_{i, m}=\varepsilon_{i+K_{m-1}^{1}+1}^{n, 1}$ and $\dot{\varepsilon}_{i, m}=\varepsilon_{K_{m-1}^{1}+1}^{n, 1}$ for $i \leq k_{m}^{1}$ and $\tilde{\varepsilon}_{i, m}=\varepsilon_{i-k_{m}^{1}+K_{m-1}^{2}+1}^{n, 2}$ and $\dot{\varepsilon}_{i, m}=\varepsilon_{K_{m-1}^{2}+1}^{n, 2}$ for $i>k_{m}^{1}$. Moreover, let

$$
\begin{aligned}
& \tilde{Z}_{1, m}=\left(\left(\left(\tilde{b}_{m, *}^{1} \cdot\left(W_{S_{i}^{n, 1}}-W_{S_{i-1}^{n, 1}}\right)\right)_{i=K_{m-1}^{1}+2}^{K_{m}^{1}}\right)^{\top},\left(\left(\tilde{b}_{m, *}^{2} \cdot\left(W_{S_{j}^{n, 2}}-W_{S_{j-1}^{n, 2}}\right)\right)_{j=K_{m-1}^{2}+2}^{K_{m}^{2}}\right)^{\top}\right)^{\top}, \\
& \tilde{Z}_{2, m}=\left(\left(\left(\varepsilon_{i}^{n, 1}-\varepsilon_{i-1}^{n, 1}\right)_{i=K_{m-1}^{1}+2}^{K_{m}^{1}}\right)^{\top},\left(\left(\varepsilon_{j}^{n, 2}-\varepsilon_{j-1}^{n, 2}\right)_{j=K_{m-1}^{2}+2}^{K_{m}^{2}}\right)^{\top}\right)^{\top}
\end{aligned}
$$

and $\tilde{S}_{1, m_{\vartheta} *}=\tilde{S}_{m, *}-M_{m, *}$.

Let $\tilde{U}_{1, m, *}$ be an orthogonal matrix and let $\Lambda_{1, m, *}$ be a diagonal matrix satisfying $\tilde{U}_{1, m, *} \tilde{S}_{1, m, *} \tilde{U}_{1, m, *}^{\top}=\Lambda_{1, m, *}$. Then since $\left.\tilde{Z}_{1, m}\right|_{\mathcal{G}_{s_{m-1}}} \sim N\left(0, \tilde{S}_{1, m, *}\right)$, we have $\left.\tilde{U}_{1, m, *} \tilde{Z}_{1, m}\right|_{\mathcal{G}_{s_{m-1}}} \sim N\left(0, \Lambda_{1, m, *}\right)$. Therefore, for any $q \in \mathbb{N}$ and $1 \leq j_{1}, \ldots, j_{2 q} \leq k_{m}^{1}+k_{m}^{2}$, we obtain

$$
E_{m}\left[\prod_{k=1}^{2 q}\left(\tilde{U}_{1, m, *} \tilde{Z}_{1, m}\right)_{j_{k}}\right]=\sum_{\left(l_{2 k-1}, l_{2 k}\right)_{k=1}^{q}} \prod_{k=1}^{q}\left(\Lambda_{1, m, *}\right)_{l_{2 k-1}, l_{2 k}},
$$

where the summation on the right-hand side is over all $q$-pairs $\left(l_{2 k-1}, l_{2 k}\right)_{k=1}^{q}$ of variables $j_{1}, \ldots, j_{2 q}$.

1. Let $\phi(A, B)_{i_{1}, \ldots, i_{4}}=\left(A_{i_{1}, i_{2}} B_{i_{3}, i_{4}}+A_{i_{1}, i_{3}} B_{i_{2}, i_{4}}+A_{i_{1}, i_{4}} B_{i_{2}, i_{3}}+A_{i_{2}, i_{3}} B_{i_{1}, i_{4}}+A_{i_{2}, i_{4}} B_{i_{1}, i_{3}}+\right.$ $\left.A_{i_{3}, i_{4}} B_{i_{1}, i_{2}}\right) / 2$ for square matrices $A$ and $B$ of the same size, and $\delta_{i_{1}}, \ldots, i_{q}$ be a $\{0,1\}$-valued function that is equal to 1 if and only if $i_{1}=\cdots=i_{q}$. Then we have

$$
\begin{aligned}
& E_{m}\left[\left(\tilde{Z}_{2, m}^{\top} \mathbf{S} \tilde{Z}_{2, m}\right)^{2}\right] \\
& \quad=E_{m}\left[\left(\left(\mathbf{A}_{m} \tilde{Z}_{2, m}\right)^{\top} \hat{\mathbf{S}}\left(\mathbf{A}_{m} \tilde{Z}_{2, m}\right)\right)^{2}\right]=\sum_{i_{1}, \ldots, i_{4}} \hat{\mathbf{S}}_{i_{1}, i_{2}} \hat{\mathbf{S}}_{i_{3}, i_{4}} E_{m}\left[\prod_{j=1}^{4}\left(\tilde{\varepsilon}_{i_{j}, m}-\dot{\varepsilon}_{i_{j}, m}\right)\right]
\end{aligned}
$$




$$
\begin{aligned}
= & \sum_{i_{1}, \ldots, i_{4}} \hat{\mathbf{S}}_{i_{1}, i_{2}} \hat{\mathbf{S}}_{i_{3}, i_{4}}\left\{\phi\left(\mathbf{M}_{1}, \mathbf{M}_{1}\right)_{i_{1}, \ldots, i_{4}}+\left(E\left[\left(\dot{\varepsilon}_{i_{1}, m}\right)^{4}\right]-3 E\left[\left(\dot{\varepsilon}_{i_{1}, m}\right)^{2}\right]^{2}\right)\right. \\
& \times 1_{\left\{\max _{1 \leq j \leq 4} i_{j} \leq k_{m}^{1} \text { or } \min _{1 \leq j \leq 4} i_{j}>k_{m}^{1}\right\}} \\
& \left.+2 \phi\left(\mathbf{M}_{1}, \mathbf{M}_{2}\right)_{i_{1}, \ldots, i_{4}}+\phi\left(\mathbf{M}_{2}, \mathbf{M}_{2}\right)_{i_{1}, \ldots, i_{4}}+\left(E\left[\left(\tilde{\varepsilon}_{i_{1}, m}\right)^{4}\right]-3 E\left[\left(\tilde{\varepsilon}_{i_{1}, m}\right)^{2}\right]^{2}\right) \delta_{i_{1}, i_{2}, i_{3}, i_{4}}\right\},
\end{aligned}
$$

where $\mathbf{M}_{1}=\operatorname{diag}\left(v_{1, *} \mathbf{1}, v_{2, *} \mathbf{1}\right)$ and $\mathbf{M}_{2}=\operatorname{diag}\left(v_{1, *} \mathcal{E}, v_{2, *} \mathcal{E}\right)$.

Hence, we obtain

$$
\begin{aligned}
& E_{m}\left[\left(\tilde{Z}_{m}^{\top} \mathbf{S} \tilde{Z}_{m}\right)^{2}\right] \\
& =E_{m}\left[\left(\tilde{Z}_{2, m}^{\top} \mathbf{S} \tilde{Z}_{2, m}\right)^{2}\right]+\sum_{i_{1}, \ldots, i_{4}} \mathbf{S}_{i_{1}, i_{2}} \mathbf{S}_{i_{3}, i_{4}}\left(\phi\left(\tilde{S}_{1, m, *}, \tilde{S}_{1, m, *}\right)+2 \phi\left(\tilde{S}_{1, m, *}, M_{m, *}\right)\right)_{i_{1}, \ldots, i_{4}} \\
& =2 \operatorname{tr}\left(\tilde{S}_{m, *} \mathbf{S} \tilde{S}_{m, *} \mathbf{S}\right)+\operatorname{tr}\left(\tilde{S}_{m, *} \mathbf{S}\right)^{2}+\sum_{j=1}^{2}\left(E\left[\left(\varepsilon_{K_{m-1}^{j, j}}^{n, j}\right)^{4}\right]-3 v_{j, *}^{2}\right) \operatorname{tr}\left(\hat{\mathbf{S}} \mathcal{E}_{(j)} \hat{\mathbf{S}} \mathcal{E}_{(j)}\right) \\
& \quad+\sum_{i} C_{n, i}\left|\hat{\mathbf{S}}_{i i}\right|^{2}
\end{aligned}
$$

by (A.5), where $C_{n, i}=E\left[\left(\tilde{\varepsilon}_{i, m}\right)^{4}\right]-3 E\left[\left(\tilde{\varepsilon}_{i, m}\right)^{2}\right]^{2}$ and $\mathcal{E}_{(j)}$ is a $\left(k_{m}^{1}+k_{m}^{2}\right) \times\left(k_{m}^{1}+k_{m}^{2}\right)$ matrix with elements $\left(\mathcal{E}_{(1)}\right)_{k l}=1_{\left\{k \leq k_{m}^{1}\right\}} 1_{\left\{l \leq k_{m}^{1}\right\}}$ and $\left(\mathcal{E}_{(2)}\right)_{k l}=1_{\left\{k>k_{m}^{1}\right\}} 1_{\left\{l>k_{m}^{1}\right\}}$.

Lemma A.7 and (A.1) yield

$$
\begin{aligned}
& \sum_{i} C_{n, i}\left|\hat{\mathbf{S}}_{i i}\right|^{2} \leq C \sup _{i} C_{n, i} b_{n}^{-1} \bar{k}_{n}\left\|\tilde{S}_{m, *} \mathbf{S} \tilde{S}_{m, *}\right\| \operatorname{tr}\left(\left(\mathbf{A}_{m}^{\top}\right)^{-1} \tilde{S}_{m, *}^{-2} \mathbf{A}_{m}^{-1}\right) \\
& \leq \bar{R}_{n}\left(b_{n}^{-2} k_{n}\right) \operatorname{tr}\left(\left(\begin{array}{cc}
M_{1, m} & 0 \\
0 & M_{2, m}
\end{array}\right) \tilde{S}_{m, *}^{-2}\right) \\
& \leq \bar{R}_{n}\left(b_{n}^{-2} k_{n}\right) \operatorname{tr}\left(\tilde{S}_{m, *}^{-1}\right)=\bar{R}_{n}\left(b_{n}^{-3 / 2} k_{n}^{2}\right)=\bar{R}_{n}(1) .
\end{aligned}
$$

Here, we used the fact that diagonal elements $\left(\tilde{S}_{m, *}^{-2}\right)_{11}$ and $\left(\tilde{S}_{m, *}^{-2}\right)_{k_{m}^{1}+1, k_{m}^{1}+1}$ are positive since $\tilde{S}_{m, *}^{-2}$ is positive definite.

Moreover, the assumptions and Lemmas A.1, A.4 and A.7 yield

$$
\begin{aligned}
\operatorname{tr}\left(\hat{\mathbf{S}} \mathcal{E}_{(1)} \hat{\mathbf{S}} \mathcal{E}_{(1)}\right) & \leq \operatorname{tr}\left(\hat{\mathbf{S}}\left(\begin{array}{cc}
\mathbf{1}+\mathcal{E} & 0 \\
0 & 0
\end{array}\right) \hat{\mathbf{S}} \mathcal{E}_{(1)}\right) \\
& \leq C \operatorname{tr}\left(\tilde{S}_{m, *}^{-1 / 2} \mathbf{A}_{m}^{-1} \mathcal{E}_{(1)}\left(\mathbf{A}_{m}^{\top}\right)^{-1} \tilde{S}_{m, *}^{-1 / 2}\right)\left\|\tilde{S}_{m, *}^{1 / 2} \mathbf{S}\left(\begin{array}{cc}
M_{1, m} & 0 \\
0 & 0
\end{array}\right) \mathbf{S} \tilde{S}_{m, *}^{1 / 2}\right\| \\
& \leq C b_{n}^{-2} r_{n} \underline{\mathrm{r}}_{n}^{-3}\left(\tilde{S}_{m, *}^{-1}\right)_{11} \leq C b_{n}^{-2} r_{n} \underline{\mathrm{r}}_{n}^{-3}\left(M_{m, *}^{-1}\right)_{11}=\bar{R}_{n}(1)
\end{aligned}
$$

since $\left(M_{m, *}^{-1}\right)_{11} \leq v_{1, *}^{-1}$ by $(4.5)$. 
(A.6)-(A.8) and similar estimates for $\operatorname{tr}\left(\hat{\mathbf{S}} \mathcal{E}_{(2)} \hat{\mathbf{S}} \mathcal{E}_{(2)}\right)$ yield $E_{m}\left[\left(\tilde{Z}_{m}^{\top} \mathbf{S} \tilde{Z}_{m}\right)^{2}\right]=2 \operatorname{tr}\left(\left(\mathbf{S} \tilde{S}_{m, *}\right)^{2}\right)+$ $\operatorname{tr}\left(\mathbf{S} \tilde{S}_{m, *}\right)^{2}+\bar{R}_{n}(1)$.

We next prove the estimate for $E_{m}\left[\left|\tilde{Z}_{m}^{\top} \mathbf{S} \tilde{Z}_{m}\right|^{q}\right]$. Let $p \in \mathbb{N}$ satisfy $q \leq 2 p$. Then it is sufficient to show that $E_{m}\left[\left(\tilde{Z}_{m}^{\top} \mathbf{S} \tilde{Z}_{m}\right)^{2 p}\right]=\bar{R}_{n}\left(b_{n}^{-2 p} k_{n}^{4 p}\right)$.

Note that

$$
E_{m}\left[\left(\tilde{Z}_{2, m}^{\top} \mathbf{S} \tilde{Z}_{2, m}\right)^{2 p}\right]=\sum_{i_{1}, \ldots, i_{4 p}} \hat{\mathbf{S}}_{i_{1}, i_{2}} \cdots \hat{\mathbf{S}}_{i_{4 p-1}, i_{4 p}} E_{m}\left[\prod_{j=1}^{4 p}\left(\tilde{\varepsilon}_{i_{j}, m}-\dot{\varepsilon}_{i_{j}, m}\right)\right],
$$

and there exist $\{0,1\}$-valued maps $\left\{A_{l, m}\right\}_{l}$, constants $C_{l}$, positive integers $\left\{q_{l}^{\prime}\right\}$ not greater than $4 p$ and injections $\left\{\iota_{l}\right\}$ such that $E_{m}\left[\prod_{j=1}^{4 p}\left(\tilde{\varepsilon}_{i_{j}, m}-\dot{\varepsilon}_{i_{j}, m}\right)\right]=\sum_{l} C_{l} A_{l, m}\left(i_{l_{l}(1)}, \ldots, i_{l_{l}\left(q_{l}^{\prime}\right)}\right)$ and $\sum_{j_{1}, \ldots, j_{q_{l}^{\prime}}} A_{l, m}\left(j_{1}, \ldots, j_{q_{l}^{\prime}}\right)=\bar{R}_{n}\left(k_{n}^{\left[q_{l}^{\prime} / 2\right]}\right)$ for any $l$. Then Lemma A.8 yields

$$
E_{m}\left[\left(\tilde{Z}_{2, m}^{\top} \mathbf{S} \tilde{Z}_{2, m}\right)^{2 p}\right]=\bar{R}_{n}\left(b_{n}^{-2 p} k_{n}^{4 p}\right)
$$

Moreover, (A.5) yields

$$
E_{m}\left[\left(\tilde{Z}_{1, m}^{\top} \mathbf{S} \tilde{Z}_{1, m}\right)^{2 p}\right] \leq C_{p} \sum_{\substack{\gamma=\left(\gamma_{1}, \ldots, \gamma_{L}\right) ; \\ L \in \mathbb{N}, \gamma_{k} \geq 1, \sum_{k} \gamma_{k}=2 p}} \prod_{k} \operatorname{tr}\left(\left(\mathbf{S} \tilde{S}_{1, m, *}\right)^{\gamma_{k}}\right)=\bar{R}_{n}\left(b_{n}^{-p} k_{n}^{2 p}\right)
$$

Furthermore, by calculating the expectation of $\tilde{Z}_{1, m}$ and using (A.5) and Lemma A.7, we have

$$
\begin{aligned}
E_{m}\left[\left(\tilde{Z}_{1, m}^{\top} \mathbf{S} \tilde{Z}_{2, m}\right)^{2 p}\right] & =\left(\sum_{\left(l_{2 k-1}, l_{2 k}\right)_{k=1}^{q}} 1\right) E_{m}\left[\left(\tilde{Z}_{2, m}^{\top} \mathbf{S} \tilde{S}_{1, m, *} \mathbf{S} \tilde{Z}_{2, m}\right)^{p}\right] \\
& \leq(2 p-1) ! !\left\|M_{m, *} \mathbf{S} \tilde{S}_{1, m, *} \mathbf{S} M_{m, *}\right\|^{p} E_{m}\left[\left(\tilde{Z}_{2, m}^{\top} M_{m, *}^{-2} \tilde{Z}_{2, m}\right)^{p}\right] \\
& =\bar{R}_{n}\left(b_{n}^{-p} k_{n}^{2 p}\right) .
\end{aligned}
$$

Then we obtain $E_{m}\left[\left(\tilde{Z}_{m}^{\top} \mathbf{S} \tilde{Z}_{m}\right)^{2 p}\right]=\bar{R}_{n}\left(b_{n}^{-2 p} k_{n}^{4 p}\right)$.

For the estimate of $E_{m}\left[\left(\tilde{Z}_{m}^{\top} \mathbf{S} \tilde{Z}_{m}\right)^{4}\right]$, we have $E_{m}\left[\left(\tilde{Z}_{1, m}^{\top} \mathbf{S} \tilde{Z}_{1, m}\right)^{4}\right]=\bar{R}_{n}\left(b_{n}^{-2} k_{n}^{4}\right)$ and $E_{m}\left[\left(\tilde{Z}_{1, m}^{\top} \mathbf{S} \tilde{Z}_{2, m}\right)^{4}\right]=\bar{R}_{n}\left(b_{n}^{-2} k_{n}^{4}\right)$ by the above results. Moreover, we have

$$
E_{m}\left[\left(\tilde{Z}_{2, m}^{\top} \mathbf{S} \tilde{Z}_{2, m}\right)^{4}\right]=\sum_{i_{1}, \ldots, i_{8}} \prod_{k=1}^{4}\left(\left(\mathbf{A}_{m}^{\top}\right)^{-1} \mathbf{S} \mathbf{A}_{m}^{-1}\right)_{i_{2 k-1}, i_{2 k}} E_{m}\left[\prod_{k=1}^{8}\left(\tilde{\varepsilon}_{i_{k}, m}-\dot{\varepsilon}_{i_{k}, m}\right)\right]
$$


and there exist $\{0,1\}$-valued maps $\left\{A_{l}^{\prime}\right\}_{l}$, constants $C_{l}^{\prime}$, positive integers $\left\{q_{l}^{\prime \prime}\right\}$ not greater than 8 and injections $\left\{\iota_{l}^{\prime}\right\}$ such that $\sum_{j_{1}, \ldots, j_{q_{l}^{\prime \prime}}} A_{l}^{\prime}\left(j_{1}, \ldots, j_{q_{l}^{\prime \prime}}\right)=\bar{R}_{n}\left(k_{n}^{\left[q_{l}^{\prime \prime} / 2\right] \wedge 3}\right)$ and

$$
E_{m}\left[\prod_{j=1}^{8}\left(\tilde{\varepsilon}_{i_{j}, m}-\dot{\varepsilon}_{i_{j}, m}\right)\right]=\sum_{\left(l_{2 k-1}, l_{2 k}\right)_{k=1}^{4}} \prod_{k=1}^{4} \delta_{l_{2 k-1}, l_{2 k}}+\sum_{l} C_{l} A_{l}^{\prime}\left(i_{l_{l}(1)}, \ldots, i_{l_{l}\left(q_{l}^{\prime}\right)}\right)
$$

where the summation in the first term of the right-hand side is over all 4-pairs $\left(l_{2 k-1}, l_{2 k}\right)_{k=1}^{4}$ of variables $i_{1}, \ldots, i_{8}$.

Then (A.11), (A.12) and Lemma A.8 yield

$$
\begin{aligned}
E_{m}\left[\left(\tilde{Z}_{2, m}^{\top} \mathbf{S} \tilde{Z}_{2, m}\right)^{4}\right] & =\sum_{i_{1}, \ldots, i_{8}} \prod_{k=1}^{4}\left(\left(\mathbf{A}_{m}^{-1}\right)^{\top} \mathbf{S A}_{m}^{-1}\right)_{i_{2 k-1}, i_{2 k}} \sum_{\left(l_{2 k-1}, l_{2 k}\right)_{k=1}^{4}} \prod_{k=1}^{4} \delta_{l_{2 k-1}, l_{2 k}}+\bar{R}_{n}\left(b_{n}^{-4} k_{n}^{7}\right) \\
& =\bar{R}_{n}\left(\left(b_{n}^{-4} k_{n}^{7}\right) \vee\left(b_{n}^{-2} k_{n}^{4}\right)\right) .
\end{aligned}
$$

2. Let $\left\{\tilde{I}_{i, m}\right\}_{i=1}^{k_{m}^{1}+k_{m}^{2}}$ be defined by $\tilde{I}_{i, m}=I_{i, m}^{1}$ for $1 \leq i \leq k_{m}^{1}$ and $\tilde{I}_{i, m}=I_{i-k_{m}^{1}, m}^{2}$ for $k_{m}^{1}<i \leq$ $k_{m}^{1}+k_{m}^{2}$. Since $\left(Z_{m}-\tilde{Z}_{2, m} \pm \tilde{Z}_{1, m}\right)_{i}=\int_{\tilde{I}_{i, m}}\left(b_{t, *}^{\mathbf{k}(i)} \pm \tilde{b}_{m, *}^{\mathbf{k}(i)}\right) d W_{t}+\mu_{s_{m-1}}^{\mathbf{k}(i)}\left|\tilde{I}_{i, m}\right|+\int_{\tilde{I}_{i, m}}\left(\mu_{t}^{\mathbf{k}(i)}-\right.$ $\left.\mu_{s_{m-1}}^{\mathbf{k}(i)}\right) d t$, we have $\left(Z_{m}-\tilde{Z}_{m}\right)^{\top} \mathbf{S}\left(Z_{m}+\tilde{Z}_{m}\right)=\Psi_{m, 1}+\Psi_{m, 2}+\Psi_{m, 3}$, where

$$
\begin{aligned}
\Psi_{m, 1}= & 2\left(Z_{m}-\tilde{Z}_{m}\right)^{\top} \mathbf{S} \tilde{Z}_{2, m}+\sum_{k=1}^{2} \sum_{i, j} \mathbf{S}_{i, j} \mu_{s_{m-1}}^{\mathbf{k}(i)}\left|\tilde{I}_{i, m}\right| \int_{\tilde{I}_{j, m}}\left(b_{t, *}^{\mathbf{k}(j)}+(-1)^{k} \tilde{b}_{m, *}^{\mathbf{k}(j)}\right) d W_{t} \\
& +\sum_{k=1}^{2} \sum_{i, j} \mathbf{S}_{i, j} \int_{\tilde{I}_{i, m}}\left(b_{t, *}^{\mathbf{k}(i)}+(-1)^{k} \tilde{b}_{m, *}^{\mathbf{k}(i)}\right) \int_{\tilde{I}_{j, m} \cap[0, t)}\left(b_{s, *}^{\mathbf{k}(j)}+(-1)^{k-1} \tilde{b}_{m, *}^{\mathbf{k}(j)}\right) d W_{s} d W_{t},
\end{aligned}
$$

$E_{m}\left[\Psi_{m, 2}\right]=0, \Psi_{m, 2}=\bar{R}_{n}\left(b_{n}^{-1} k_{n}^{3 / 2}\right)$ and $\Psi_{m, 3}=\bar{R}_{n}\left(b_{n}^{-3 / 2} k_{n}^{2}\right)$. Here we used the facts that

$$
\left|x^{\top} A y\right| \leq\|A\||x \| y|
$$

for vectors $x, y$ and a matrix $A$,

$$
\left|E_{m}[\operatorname{tr}(\mathbf{S} \mathcal{D})]\right|=\left|\operatorname{tr}\left(\mathbf{S} E_{m}[\mathcal{D}]\right)\right| \leq\left\|E_{m}[\mathcal{D}]\right\|\left\|\mathbf{S} \tilde{S}_{m, *}\right\| \operatorname{tr}\left(\tilde{S}_{m, *}^{-1}\right)=\bar{R}_{n}\left(b_{n}^{-3 / 2} k_{n}^{2}\right),
$$

and

$$
\begin{aligned}
E_{\Pi}\left[\bar{E}_{m}[\operatorname{tr}(\mathbf{S} \mathcal{D})]^{q}\right] & \leq C E_{\Pi}\left[E_{m}\left[\|\mathcal{D}\|^{q}\right]\left\|\tilde{S}_{m, *} \mathbf{S}\right\|^{q} \operatorname{tr}\left(\tilde{S}_{m, *}^{-1}\right)^{q}\right] \\
& \leq C E_{\Pi}\left[\bar{R}_{n}\left(b_{n}^{3 / 2 q} \ell_{n}^{-q}\right) \bar{R}_{n}\left(b_{n}^{-q} \ell_{n}^{-q / 2}\right)\right]
\end{aligned}
$$

for any $q \geq 1$, where $\mathcal{D}=\left(\mathcal{D}_{i j}\right)_{i j}$ and $\mathcal{D}_{i j}=\int_{\tilde{I}_{i, m} \cap \tilde{I}_{j, m}}\left(b_{t, *}^{\mathbf{k}(i)}+\tilde{b}_{m, *}^{\mathbf{k}(i)}\right)\left(b_{t, *}^{\mathbf{k}(j)}-\tilde{b}_{m, *}^{\mathbf{k}(j)}\right) d t$. 
Then the Burkholder-Davis-Gundy inequality yields

$$
\begin{aligned}
E_{\Pi} & {\left[\left|\sum_{m}\left(Z_{m}-\tilde{Z}_{m}\right)^{\top} \mathbf{S}\left(Z_{m}+\tilde{Z}_{m}\right)\right|^{q}\right] } \\
\leq & \leq E_{\Pi}\left[\left(\sum_{m}\left(\Psi_{m, 1}+\Psi_{m, 2}\right)^{2}\right)^{\frac{q}{2}}\right]+\bar{R}_{n}\left(b_{n}^{-\frac{q}{2}} k_{n}^{q}\right) \\
& \leq C E_{\Pi}\left[\left(\sum_{m} E_{m}\left[\Psi_{m, 1}^{2}\right]\right)^{\frac{q}{2}}\right]+C E_{\Pi}\left[\left(\sum_{m} \bar{E}_{m}\left[\Psi_{m, 1}^{2}\right]^{2}\right)^{\frac{q}{4}}\right]+\bar{R}_{n}\left(b_{n}^{-\frac{q}{2}} k_{n}^{q}\right) .
\end{aligned}
$$

Let $\dot{Z}_{m, i}=\int_{\tilde{I}_{i, m}}\left(b_{t, *}^{\mathbf{k}(i)}-\tilde{b}_{m, *}^{\mathbf{k}(i)}\right) d W_{t}$ and $\dot{Z}_{m}=\left(\dot{Z}_{m, i}\right)_{i}$, then we obtain

$$
\begin{aligned}
\Psi_{m, 1}= & 2\left(Z_{m}-\tilde{Z}_{m}\right)^{\top} \mathbf{S} \tilde{Z}_{2, m}+\dot{Z}_{m}^{\top} \mathbf{S} \dot{Z}_{m}+2 \tilde{Z}_{1, m}^{\top} \mathbf{S} \dot{Z}_{m} \\
& +\left(2 \dot{Z}_{m}+2 \tilde{Z}_{1, m}\right)^{\top} \mathbf{S}\left(\mu_{s_{m-1}}^{\mathbf{k}(i)}\left|\tilde{I}_{i, m}\right|\right)_{i}+\bar{R}_{n}\left(b_{n}^{-1} k_{n}^{3 / 2}\right) .
\end{aligned}
$$

Moreover, for any $p \in \mathbb{N}$, Lemma A.7, (A.13), (A.9) and the Cauchy-Schwarz inequality yield

$$
\begin{aligned}
E_{m} & {\left[\left(2\left(Z_{m}-\tilde{Z}_{m}\right)^{\top} \mathbf{S} \tilde{Z}_{2, m}+\left(2 \dot{Z}_{m}+2 \tilde{Z}_{1, m}\right)^{\top} \mathbf{S}\left(\mu_{s_{m-1}}^{\mathbf{k}(i)}\left|\tilde{I}_{i, m}\right|\right)_{i}\right)^{2 p}\right] } \\
\leq & C E_{m}\left[\left\|\tilde{S}_{m, *} \mathbf{S}\left(Z_{m}-\tilde{Z}_{m}\right)\left(Z_{m}-\tilde{Z}_{m}\right)^{\top} \mathbf{S} \tilde{S}_{m, *}\right\|^{2 p}\right]^{1 / 2} E_{m}\left[\left(\tilde{Z}_{2, m}^{\top} \tilde{S}_{m, *}^{-2} \tilde{Z}_{2, m}\right)^{2 p}\right]^{1 / 2} \\
& \quad+\bar{R}_{n}\left(\left(b_{n} k_{n} b_{n}^{-1 / 2} b_{n}^{-1}\right)^{2 p}\right) \\
= & \bar{R}_{n}\left(\left(b_{n}^{-1} \ell_{n}^{-1} k_{n}\right)^{p} k_{n}^{2 p}\right)+\bar{R}_{n}\left(b_{n}^{-p} k_{n}^{2 p}\right)=\bar{R}_{n}\left(b_{n}^{-2 p} k_{n}^{4 p}\right) .
\end{aligned}
$$

We can rewrite $\dot{Z}_{m, i}=\mathbf{L}_{i}^{1}+\mathbf{L}_{i}^{2}+\mathbf{L}_{i}^{3}+\bar{R}_{n}\left(b_{n}^{-1 / 2} \ell_{n}^{-3 / 2}\right)$, where $\mathbf{L}_{i}^{1}=\sum_{j} \xi_{j}^{1} \int_{\tilde{I}_{i, m}}(t-$ $\left.s_{m-1}\right) d W_{t}^{j}, \mathbf{L}_{i}^{2}=\sum_{j, k} \xi_{j, k}^{2} \int_{\tilde{I}_{i, m}}\left(W_{t}^{k}-W_{s_{m-1}}^{k}\right) d W_{t}^{j}$ and $\mathbf{L}_{i}^{3}=\sum_{j, k, l} \xi_{j, k, l}^{3} \int_{\tilde{I}_{i, m}} \int_{s_{m-1}}^{t}\left(W_{s}^{l}-\right.$ $\left.W_{s_{m-1}}^{l}\right) d W_{s}^{k} d W_{t}^{j}$ for some $\mathcal{G}_{s_{m-1}}$-measurable random variables $\xi_{j}^{1}, \xi_{j, k}^{2}$, and $\xi_{j, k, l}^{3}$ with bounded moments.

Let $\mathbf{L}^{j}=\left(\mathbf{L}_{i}^{j}\right)_{i}$. Then, Lemma A.7 and (A.10) yield

$$
\begin{aligned}
& E_{m}\left[\left(\dot{Z}_{m}^{\top} \mathbf{S} \dot{Z}_{m}+2 \tilde{Z}_{1, m}^{\top} \mathbf{S} \dot{Z}_{m}\right)^{2 p}\right] \\
& \leq C\left\|\tilde{S}_{m, *}^{1 / 2} \mathbf{S} \tilde{S}_{m, *}^{1 / 2}\right\|^{2 p}\left(E_{m}\left[\left(\left(\mathbf{L}^{2}\right)^{\top} \tilde{S}_{m, *}^{-1} \mathbf{L}^{2}\right)^{2 p}\right]+\bar{R}_{n}\left(\left(b_{n} k_{n} b_{n}^{-1} \ell_{n}^{-2}\right)^{2 p}\right)\right) \\
& \quad+C \sum_{j=1}^{3} E_{m}\left[\left(\tilde{Z}_{1, m}^{\top} \mathbf{S} \mathbf{L}^{j}\right)^{2 p}\right] \\
& \quad+E_{m}\left[\left\|\tilde{S}_{m, *} \mathbf{S}\left(\dot{Z}_{m}-\sum_{j=1}^{3} \mathbf{L}^{j}\right)^{\top}\left(\dot{Z}_{m}-\sum_{j=1}^{3} \mathbf{L}^{j}\right) \mathbf{S} \tilde{S}_{m, *}\right\|^{2 p}\right]^{1 / 2} E_{m}\left[\left(\tilde{Z}_{1, m}^{\top} \tilde{S}_{m, *}^{-2} \tilde{Z}_{1, m}\right)^{2 p}\right]^{1 / 2} \\
& \leq \bar{R}_{n}(1) E_{m}\left[\left(\left(\mathbf{L}^{2}\right)^{\top} \tilde{S}_{m, *}^{-1} \mathbf{L}^{2}\right)^{2 p}\right]+C \sum_{j=1}^{3} E_{m}\left[\left(\tilde{Z}_{1, m}^{\top} \mathbf{S} \mathbf{L}^{j}\right)^{2 p}\right]+\bar{R}_{n}\left(b_{n}^{-4 p} k_{n}^{6 p}\right) .
\end{aligned}
$$


Here we also used that $(a+b)^{\top} A(a+b) \leq 2 a^{\top} A a+2 b^{\top} A b$ for a positive definite matrix $A$ and vectors $a, b$.

Moreover, we can see that there exists a positive constant $C_{p}$ such that

$$
\begin{aligned}
& E_{m}\left[\sum_{\substack{i_{1}, \ldots, i_{2 p} \\
j_{1}, \ldots, j_{2 p}}} \prod_{k=1}^{2 p}\left(W^{p_{1, k}}\left(\tilde{I}_{i_{k}, m}\right) \int_{\tilde{I}_{j_{k}, m}}\left(W_{t}^{p_{2, k}}-W_{s_{m-1}}^{p_{2, k}}\right) d W_{t}^{p_{3, k}}\right)\right] \\
& \quad \leq C_{p} \sum_{\substack{i_{1}, \ldots, i_{2 p} \\
j_{1}, \ldots, j_{2 p}}} \sum_{\substack{\left.l_{2 q-1}, l_{2 q}\right)_{q=1}^{2 p-\alpha}, \alpha \\
q=1}}^{2 p-\alpha}\left(\prod_{q=1}^{\left.2 p-\tilde{I}_{l_{2 q-1}, m} \cap \tilde{I}_{l_{2 q, m}} \mid\right) r_{n}^{2 \alpha}\left(s_{m}-s_{m-1}\right)^{p-\alpha}}\right.
\end{aligned}
$$

for any $\left\{p_{l, k}\right\}_{1 \leq l \leq 3,1 \leq k \leq 2 p} \subset\{1,2\}$, where the second summation on the right-hand side is taken over $0 \leq \alpha \leq p$ and $(2 p-\alpha)$ disjoint pairs $\left(l_{2 q-1}, l_{2 q}\right)_{q=1}^{2 p-\alpha}$ in the variables $i_{1}, \ldots, i_{2 p}, j_{1}, \ldots, j_{2 p}$. Here we used the fact that all $6 p$ factors $\left(W^{p_{1, k}}\left(\tilde{I}_{i_{k}, m}\right), \int_{\tilde{I}_{j_{k}, m}} \cdot d W_{t}^{p_{3, k}}\right.$, $\left.\left(W_{t}^{p_{2}, k}-W_{s_{m-1}}^{p_{2, k}}\right)_{t \in \tilde{I}_{j_{k}, m}}\right)_{k=1}^{2 p}$ should be separated into $3 p$ pairs in the non-zero terms. $2 \alpha$ represents the number of pairs with the form $\left(W^{p_{1, k}}\left(\tilde{I}_{i_{k}, m}\right),\left(W_{t}^{p_{2, k^{\prime}}}-W_{s_{m-1}}^{p_{2, k^{\prime}}}\right)_{t \in \tilde{I}_{j_{k^{\prime}}, m}}\right)$ or $\left(\int_{\tilde{I}_{j_{k}, m}} \cdot d W_{t}^{p_{3, k}},\left(W_{t}^{p_{2, k^{\prime}}}-W_{s_{m-1}}^{p_{2, k^{\prime}}}\right)_{t \in \tilde{I}_{j_{k^{\prime}}, m}}\right)$. Therefore we obtain

$$
E_{m}\left[\left(\tilde{Z}_{1, m}^{\top} \mathbf{S L}^{2}\right)^{2 p}\right]=\bar{R}_{n}\left(\sum_{0 \leq \alpha \leq p}\left(b_{n}^{1 / 2} \ell_{n}^{-1}\right)^{2 p-\alpha}\left(b_{n}^{3 / 2} \ell_{n}^{-1} k_{n}\right)^{\alpha} r_{n}^{2 \alpha} \ell_{n}^{-p+\alpha}\right)=\bar{R}_{n}\left(\left(b_{n}^{-1} k_{n}^{3 / 2}\right)^{2 p}\right)
$$

(A.17) and similar arguments for $E_{m}\left[\left(\tilde{Z}_{1, m}^{\top} \mathbf{S L}^{1}\right)^{2 p}\right], E_{m}\left[\left(\tilde{Z}_{1, m}^{\top} \mathbf{S L}^{3}\right)^{2 p}\right]$ and $E_{m}\left[\left(\left(\mathbf{L}^{2}\right)^{\top} \mathbf{S L}^{2}\right)^{2 p}\right]$ yield

$$
E_{m}\left[\left(\dot{Z}_{m}^{\top} \mathbf{S} \dot{Z}_{m}+2 \tilde{Z}_{1, m}^{\top} \mathbf{S} \dot{Z}_{m}\right)^{2 p}\right]=\bar{R}_{n}\left(b_{n}^{-2 p} k_{n}^{3 p}\right) .
$$

(A.15), (A.16) and (A.18) yield

$$
E_{m}\left[\Psi_{m, 1}^{2 p}\right]=\bar{R}_{n}\left(b_{n}^{-2 p} k_{n}^{4 p}\right), \quad \text { and hence } \quad E_{\Pi}\left[\left(\sum_{m} \Psi_{m, 1}^{4}\right)^{q / 4}\right]=\bar{R}_{n}\left(\left(b_{n}^{-3} k_{n}^{7}\right)^{q / 4}\right) .
$$

(A.13), (A.15) and (A.18) yield

$$
\begin{aligned}
E_{m}\left[\Psi_{m, 1}^{2}\right] \leq & C\left\|\tilde{S}_{m, *}^{1 / 2} \mathbf{S} M_{m, *} \mathbf{S} \tilde{S}_{m, *}^{1 / 2}\right\| E_{m}\left[\left(Z_{m}-\tilde{Z}_{m}\right)^{\top} \tilde{S}_{m, *}^{-1}\left(Z_{m}-\tilde{Z}_{m}\right)\right]+\bar{R}_{n}\left(b_{n}^{-2} k_{n}^{3}\right) \\
& +\left\|\mathbf{S}\left\{E_{m}\left[\left(2 \dot{Z}_{m}+2 \tilde{Z}_{1, m}\right)_{i}\left(2 \dot{Z}_{m}+2 \tilde{Z}_{1, m}\right)_{j}\right]\right\}_{i, j} \mathbf{S}\right\| \sum_{i}\left(\mu_{s_{m-1}}^{\mathbf{k}(i)}\left|\tilde{I}_{i, m}\right|\right)^{2} \\
\leq & \bar{R}_{n}\left(b_{n}^{-3 / 2} k_{n}^{2}\right)+\bar{R}_{n}\left(b_{n}^{-2} k_{n}^{3}\right)+\bar{R}_{n}\left(b_{n} k_{n} b_{n}^{-2}\right)=\bar{R}_{n}\left(b_{n}^{-2} k_{n}^{3}\right),
\end{aligned}
$$

and therefore, we have $E_{\Pi}\left[\left(\sum_{m} E_{m}\left[\Psi_{m, 1}^{2}\right]\right)^{q / 2}\right]=\bar{R}_{n}\left(b_{n}^{-q / 2} k_{n}^{q}\right)$, which completes the proof of point 2. 
Point 3 is easily obtained by the proof of point 2 since we only need the estimate for $E_{\Pi}\left[\left(\sum_{m}\left[\Psi_{m, 1}^{2}\right]\right)\right]$ if $q=2$.

\section{A.4. An additional lemma}

Lemma A.9. Let $e_{n}$ be a sequence of positive numbers, $\mathcal{S}$ be an open set in a Euclidean space, $A_{n}(\lambda)$ and $B_{n}(\lambda)$ be sequences of positive-valued random variables, and $C_{n}(\lambda)$ be a sequence of non-negative-valued random variables for $\lambda \in \mathcal{S}$. Assume that $A_{n}(\lambda)$, $B_{n}(\lambda)$, and $C_{n}(\lambda)$ are $C^{3}$ with respect to $\lambda, e_{n} \rightarrow \infty, \sup _{0 \leq k \leq 3, \lambda \in \mathcal{S}}\left(\left|\partial_{\lambda}^{k} A_{n}\right| \vee\left|\partial_{\lambda}^{k} B_{n}\right|\right)=$ $O_{p}\left(e_{n}^{-1}\right)$, and $\sup _{0 \leq k \leq 3, \lambda \in \mathcal{S}}\left|\partial_{\lambda}^{k} C_{n}\right|=O_{p}\left(e_{n}^{-2}\right)$ as $n \rightarrow \infty, C_{n}<A_{n} B_{n}$ a.s. for any $n \in \mathbb{N}$, and $\lim _{n \rightarrow \infty}\left(e_{n}^{2}\left(A_{n} B_{n}-C_{n}\right)\right)>0$ a.s. Then

$$
\begin{aligned}
& \sup _{\lambda \in \mathcal{S}}\left|\partial_{\lambda}^{k}\left(\sum_{p=1}^{\infty} \int_{0}^{\pi} \frac{C_{n}^{p}}{f_{p}\left(A_{n}, x\right) f_{p}\left(B_{n}, x\right)} d x-\frac{\pi C_{n}}{2 P_{n} \sqrt{A_{n} B_{n}-C_{n}}}\right)\right|=O_{p}\left(e_{n}^{-\frac{3}{2}}\right), \\
& \sup _{\lambda \in \mathcal{S}}\left|\partial_{\lambda}^{k}\left(\sum_{p=1}^{\infty} \int_{0}^{\pi} \frac{C_{n}^{p} f_{1}\left(A_{n}, x\right)}{f_{p}\left(A_{n}, x\right) f_{p}\left(B_{n}, x\right)} d x-\frac{\pi C_{n}\left(A_{n}+\sqrt{A_{n} B_{n}-C_{n}}\right)}{2 P_{n} \sqrt{A_{n} B_{n}-C_{n}}}\right)\right| \\
& =O_{p}\left(e_{n}^{-\frac{5}{2}}\right), \\
& \sup _{\lambda \in \mathcal{S}}\left|\partial_{\lambda}^{k}\left(\sum_{p=1}^{\infty} \frac{1}{p} \int_{0}^{\pi} \frac{C_{n}^{p}}{f_{p}\left(A_{n}, x\right) f_{p}\left(B_{n}, x\right)} d x-\pi\left(\sqrt{A_{n}}+\sqrt{B_{n}}\right)+\pi P_{n}\right)\right| \\
& =O_{p}\left(e_{n}^{-1}\right)
\end{aligned}
$$

for $0 \leq k \leq 3$, where $f_{p}(a, x)=(a+2(1-\cos x))^{p}$ and $P_{n}=\sqrt{A_{n}+B_{n}+2 \sqrt{A_{n} B_{n}-C_{n}}}$.

Proof. An elementary calculation yields

$$
\begin{aligned}
& \sum_{p=1}^{\infty} \int_{0}^{\pi} \frac{C_{n}^{p}}{\left(A_{n}+2(1-\cos x)\right)^{p}\left(B_{n}+2(1-\cos x)\right)^{p}} d x \\
& \quad=\int_{-\infty}^{\infty}\left(1-\frac{C_{n}}{\left(A_{n}+\frac{4 t^{2}}{1+t^{2}}\right)\left(B_{n}+\frac{4 t^{2}}{1+t^{2}}\right)}\right)^{-1} \frac{C_{n}}{\left(A_{n}+\frac{4 t^{2}}{1+t^{2}}\right)\left(B_{n}+\frac{4 t^{2}}{1+t^{2}}\right)} \frac{1}{1+t^{2}} d t \\
& \quad=\int_{-\infty}^{\infty} \frac{C_{n}}{\left(A_{n}+\frac{4 t^{2}}{1+t^{2}}\right)\left(B_{n}+\frac{4 t^{2}}{1+t^{2}}\right)-C_{n}} \frac{1}{1+t^{2}} d t \\
& \quad=\int_{-\infty}^{\infty} \frac{C_{n}\left(1+t^{2}\right)}{\left(\left(A_{n}+4\right) t^{2}+A_{n}\right)\left(\left(B_{n}+4\right) t^{2}+B_{n}\right)-C_{n}\left(1+t^{2}\right)^{2}} d t \\
& \quad=\int_{-\infty}^{\infty} \frac{C_{n}\left(1+t^{2}\right)}{\left(A_{n} B_{n}+4 A_{n}+4 B_{n}-C_{n}+16\right) t^{4}+2\left(A_{n} B_{n}+2 A_{n}+2 B_{n}-C_{n}\right) t^{2}+A_{n} B_{n}-C_{n}} d t
\end{aligned}
$$


We only consider the case $\left(A_{n}-B_{n}\right)^{2}+16 C_{n}>0$ a.s. We can easily obtain the desired results for the other case with a slight modification.

Let

$$
\begin{aligned}
& \alpha_{1}=\frac{-2 A_{n}-2 B_{n}-\sqrt{4\left(A_{n}-B_{n}\right)^{2}+16 C_{n}}}{16} \text { and } \\
& \alpha_{2}=\frac{-2 A_{n}-2 B_{n}+\sqrt{4\left(A_{n}-B_{n}\right)^{2}+16 C_{n}}}{16},
\end{aligned}
$$

then we have $\alpha_{1}<\alpha_{2}<0$ by the assumptions. Moreover, we can calculate the right-hand side of (A.23) as

$$
\begin{aligned}
(1 & \left.+O_{p}\left(e_{n}^{-1}\right)\right) \int_{-\infty}^{\infty} \frac{C_{n}\left(1+t^{2}\right)}{16\left(t^{2}-\alpha_{1}\right)\left(t^{2}-\alpha_{2}\right)} d t \\
& =\left(1+O_{p}\left(e_{n}^{-1}\right)\right) \frac{2 \pi i}{16}\left[\frac{C_{n}\left(1+\alpha_{1}\right)}{2 \sqrt{-\alpha_{1}} i\left(\alpha_{1}-\alpha_{2}\right)}+\frac{C_{n}\left(1+\alpha_{2}\right)}{2 \sqrt{-\alpha_{2}} i\left(\alpha_{2}-\alpha_{1}\right)}\right] \\
& =\left(1+O_{p}\left(e_{n}^{-1}\right)\right) \frac{\pi}{16} \frac{C_{n}\left(\sqrt{-\alpha_{2}}-\sqrt{-\alpha_{1}}\right)\left(1+\sqrt{\alpha_{1} \alpha_{2}}\right)}{\sqrt{\alpha_{1} \alpha_{2}}\left(\alpha_{1}-\alpha_{2}\right)} \\
& =\frac{\pi}{16} \frac{C_{n}}{\sqrt{\alpha_{1} \alpha_{2}}\left(\sqrt{-\alpha_{1}}+\sqrt{-\alpha_{2}}\right)}+O_{p}\left(e_{n}^{-3 / 2}\right)
\end{aligned}
$$

Therefore, we obtain (A.20) with $k=0$ by noting that $16 \alpha_{1} \alpha_{2}=A_{n} B_{n}-C_{n}$ and

$$
\sqrt{x+y+\sqrt{(x-y)^{2}+4 z}}+\sqrt{x+y-\sqrt{(x-y)^{2}+4 z}}=\sqrt{2 x+2 y+4 \sqrt{x y-z}}
$$

for non-negative numbers $x, y, z$ satisfying $x y \geq z$.

We similarly have (A.20) with $1 \leq k \leq 3$ and (A.21) with $0 \leq k \leq 3$.

Furthermore, we obtain $\int_{-\infty}^{\infty}\left(1+t^{2}\right)^{-1} \log \left(t^{2}+\alpha^{2}\right) d t=2 \pi \log (\alpha+1)$ for any $\alpha>0$ by the residue theorem.

Therefore, we obtain

$$
\begin{aligned}
\partial_{\lambda}^{k} & \sum_{p=1}^{\infty} \frac{1}{p} \int_{0}^{\pi} \frac{C_{n}^{p}}{f_{p}\left(A_{n}, x\right) f_{p}\left(B_{n}, x\right)} d x \\
& =-\partial_{\lambda}^{k} \int_{-\infty}^{\infty} \frac{1}{1+t^{2}} \log \left(1-\frac{C_{n}}{\left(A_{n}+4 t^{2} /\left(1+t^{2}\right)\right)\left(B_{n}+4 t^{2} /\left(1+t^{2}\right)\right)}\right) d t \\
& =-\partial_{\lambda}^{k} \int_{-\infty}^{\infty} \frac{1}{1+t^{2}} \log \left(\frac{\left(t^{2}-\alpha_{1}\right)\left(t^{2}-\alpha_{2}\right)}{\left(t^{2}+A_{n} /\left(A_{n}+4\right)\right)\left(t^{2}+B_{n} /\left(B_{n}+4\right)\right)}\right) d t+O_{p}\left(e_{n}^{-1}\right) \\
& =-2 \pi \partial_{\lambda}^{k}\left(\log \left(1+\sqrt{-\alpha_{1}}\right)+\log \left(1+\sqrt{-\alpha_{2}}\right)-\log \left(1+\sqrt{\frac{A_{n}}{A_{n}+4}}\right)\right.
\end{aligned}
$$




$$
\begin{aligned}
& \left.-\log \left(1+\sqrt{\frac{B_{n}}{B_{n}+4}}\right)\right)+O_{p}\left(e_{n}^{-1}\right) \\
= & 2 \pi \partial_{\lambda}^{k}\left(\sqrt{A_{n}} / 2+\sqrt{B_{n}} / 2-\sqrt{-\alpha_{1}}-\sqrt{-\alpha_{2}}\right)+O_{p}\left(e_{n}^{-1}\right),
\end{aligned}
$$

which completes the proof.

\section{Acknowledgement}

The author is grateful to Yuta Koike for very useful discussions and his help on numerical analysis of quadratic covariation estimators. He had a great contribution on constructing the "cce" function in the "yuima" $\mathrm{R}$ package as well. This work was partially supported by Japan Society for the Promotion of Science KAKENHI Grant Number 15K21598, and JST PRESTO Grant Number JPMJPR15E2, Japan.

\section{References}

[1] Adams, R.A. and Fournier, J.J.F. (2003). Sobolev Spaces, 2nd ed. Pure and Applied Mathematics (Amsterdam) 140. Amsterdam: Elsevier/Academic Press. MR2424078

[2] Aït-Sahalia, Y., Fan, J. and Xiu, D. (2010). High-frequency covariance estimates with noisy and asynchronous financial data. J. Amer. Statist. Assoc. 105 1504-1517. MR2796567

[3] Barndorff-Nielsen, O.E., Hansen, P.R., Lunde, A. and Shephard, N. (2008). Designing realized kernels to measure the ex post variation of equity prices in the presence of noise. Econometrica 76 1481-1536. MR2468558

[4] Barndorff-Nielsen, O.E., Hansen, P.R., Lunde, A. and Shephard, N. (2011). Multivariate realised kernels: Consistent positive semi-definite estimators of the covariation of equity prices with noise and non-synchronous trading. J. Econometrics 162 149-169. MR2795610

[5] Bibinger, M., Hautsch, N., Malec, P. and Reiss, M. (2014). Estimating the quadratic covariation matrix from noisy observations: Local method of moments and efficiency. Ann. Statist. 42 80-114. MR3226158

[6] Billingsley, P. (1999). Convergence of Probability Measures, 2nd ed. Wiley Series in Probability and Statistics: Probability and Statistics. New York: Wiley. MR1700749

[7] Christensen, K., Kinnebrock, S. and Podolskij, M. (2010). Pre-averaging estimators of the ex-post covariance matrix in noisy diffusion models with non-synchronous data. J. Econometrics 159116 133. MR2720847

[8] Christensen, K., Podolskij, M. and Vetter, M. (2013). On covariation estimation for multivariate continuous Itô semimartingales with noise in non-synchronous observation schemes. J. Multivariate Anal. 120 59-84. MR3072718

[9] Cox, J.C., Ingersoll, J.E. Jr. and Ross, S.A. (1985). A theory of the term structure of interest rates. Econometrica 53 385-407. MR0785475

[10] Doukhan, P. and Louhichi, S. (1999). A new weak dependence condition and applications to moment inequalities. Stochastic Process. Appl. 84 313-342. MR1719345

[11] Genon-Catalot, V. and Jacod, J. (1994). Estimation of the diffusion coefficient for diffusion processes: Random sampling. Scand. J. Stat. 21 193-221. MR1292636 
[12] Gloter, A. and Jacod, J. (2001). Diffusions with measurement errors. I. Local asymptotic normality. ESAIM Probab. Stat. 5 225-242. MR1875672

[13] Gloter, A. and Jacod, J. (2001). Diffusions with measurement errors. II. Optimal estimators. ESAIM Probab. Stat. 5 243-260. MR1875673

[14] Gobet, E. (2001). Local asymptotic mixed normality property for elliptic diffusion: A Malliavin calculus approach. Bernoulli 7 899-912. MR1873834

[15] Hayashi, T. and Yoshida, N. (2005). On covariance estimation of non-synchronously observed diffusion processes. Bernoulli 11 359-379. MR2132731

[16] Hayashi, T. and Yoshida, N. (2008). Asymptotic normality of a covariance estimator for nonsynchronously observed diffusion processes. Ann. Inst. Statist. Math. 60 367-406. MR2403524

[17] Hayashi, T. and Yoshida, N. (2011). Nonsynchronous covariation process and limit theorems. Stochastic Process. Appl. 121 2416-2454. MR2822782

[18] Jacod, J. (1997). On continuous conditional Gaussian martingales and stable convergence in law. In Séminaire de Probabilités, XXXI. Lecture Notes in Math. 1655 232-246. Berlin: Springer. MR1478732

[19] Jacod, J., Li, Y., Mykland, P.A., Podolskij, M. and Vetter, M. (2009). Microstructure noise in the continuous case: The pre-averaging approach. Stochastic Process. Appl. 119 2249-2276. MR2531091

[20] Jeganathan, P. (1982). On the asymptotic theory of estimation when the limit of the log-likelihood ratios is mixed normal. Sankhyā Ser. A 44 173-212. MR0688800

[21] Jeganathan, P. (1983). Some asymptotic properties of risk functions when the limit of the experiment is mixed normal. Sankhya Ser. A 45 66-87. MR0749355

[22] Malliavin, P. and Mancino, M.E. (2002). Fourier series method for measurement of multivariate volatilities. Finance Stoch. 6 49-61. MR1885583

[23] Malliavin, P. and Mancino, M.E. (2009). A Fourier transform method for nonparametric estimation of multivariate volatility. Ann. Statist. 37 1983-2010. MR2533477

[24] Ogihara, T. (2015). Local asymptotic mixed normality property for nonsynchronously observed diffusion processes. Bernoulli 21 2024-2072. MR3378458

[25] Ogihara, T. and Yoshida, N. (2014). Quasi-likelihood analysis for nonsynchronously observed diffusion processes. Stochastic Process. Appl. 124 2954-3008. MR3217430

[26] Podolskij, M. and Vetter, M. (2009). Estimation of volatility functionals in the simultaneous presence of microstructure noise and jumps. Bernoulli 15 634-658. MR2555193

[27] Uchida, M. (2010). Contrast-based information criterion for ergodic diffusion processes from discrete observations. Ann. Inst. Statist. Math. 62 161-187. MR2577445

[28] Uchida, M. and Yoshida, N. (2013). Quasi likelihood analysis of volatility and nondegeneracy of statistical random field. Stochastic Process. Appl. 123 2851-2876. MR3054548

[29] Yoshida, N. (2006). Polynomial type large deviation inequalities and convergence of statistical random fields. ISM Research Memorandum 1021.

[30] Yoshida, N. (2011). Polynomial type large deviation inequalities and quasi-likelihood analysis for stochastic differential equations. Ann. Inst. Statist. Math. 63 431-479. MR2786943

[31] Zhang, L., Mykland, P.A. and Aït-Sahalia, Y. (2005). A tale of two time scales: Determining integrated volatility with noisy high-frequency data. J. Amer. Statist. Assoc. 100 1394-1411. MR2236450

Received December 2015 and revised April 2017 